\title{
Topics in Matroid Union
}

by

Ali M Hameed

A thesis

submitted to Victoria University of Wellington

in fulfilment of the

requirements for the degree of

Master of Science

in Mathematics.

Victoria University of Wellington

2008 
VICTORIA UNIVERSITY OF WELLINGTON 


\begin{abstract}
The operation of matroid union was introduced by Nash-Williams in 1966. A matroid is indecomposable if it cannot be written in the form $M=M_{1} \vee M_{2}$, where $r\left(M_{1}\right), r\left(M_{2}\right)>0$. In 1971 Welsh posed the problem of characterizing indecomposable matroids, this problem has turned out to be extremely difficult. As a partial solution towards its progress, Cunningham characterized binary indecomposable matroids in 1977. In this thesis we present numerous results in topics of matroid union. Those include a link between matroid connectivity and matroid union, such as the implication of having a 2-separation in the matroid union, and under what conditions is the union 3connected. We also identify which elements in binary and ternary matroids are non-fixed. Then we create a link between having non-fixed elements in binary and ternary matroids and the decomposability of such matroids, and the effect of removing non-fixed elements from binary and ternary matroids. Moreover, we show results concerning decomposable 3-connected ternary matroids, such as what essential property every decomposable 3-connected ternary matroid must have, how to compose a ternary matroid, and what a 3-connected ternary matroid decomposes into. We also give an alternative statement and an alternative proof of Cunningham's theorem from the perspective of fixed and non-fixed elements.
\end{abstract}




\section{Acknowledgments}

The researcher wishes to sincerely thank Professor Geoff Whittle for his supervision of this study and for his vital support, guidance, inspiration and encouragement during the course of this work. Gratitude also goes to Professor Andras Recski, Professor James Oxley for pointing me to some important papers, and to Dr Steven Archer and Dr Dillon Mayhew for helping me resolve $\mathrm{LAT}_{\mathrm{E}} \mathrm{X}$ issues. I would also like to express my appreciation and gratitude to my wife Hiba, and my parents, who have given great support and encouragement, and constant caring, without which, my task would have been much more difficult. 


\section{List of Figures}

1.1 The Fano matroid $\ldots \ldots \ldots \ldots$

1.2 Series connection and deletion. . . . . . . . . . . . . 11

1.3 Parallel connection and contraction. . . . . . . . . . . 12

1.4 General view of 1-separation and 2-separations. . . . . . . . . 14

1.5 Examples of 1- and 2-separations. . . . . . . . . . . 15

2.1 An example of a matroid union. . . . . . . . . . . 20

$2.2 \quad M_{1} \vee M_{2}$ is induced from $M_{1} \oplus M_{2}$ by $\triangle \ldots \ldots \ldots 24$

2.3 Contracting an intersection element. . . . . . . . . . . 30

2.4 Contracting an intersection element may give an indecomposable matroid. . . . . . . . . . . . . . . . . . . . 31

$3.1 \quad M^{\prime}=P\left(M_{A}, M_{B}\right) \ldots \ldots \ldots \ldots \ldots \ldots \ldots$

$3.2 \quad N_{1}^{\prime}=P\left(N_{1 A}, N_{1 B}\right) \ldots \ldots \ldots \ldots \ldots \ldots \ldots \ldots$

$3.3 \quad N_{2}^{\prime}=\left(N_{2} \mid A\right) \oplus\left(N_{2} \mid B\right) \oplus U_{0,1} \ldots \ldots \ldots \ldots \ldots \ldots \ldots$

$3.4 \quad S\left(M_{1}, M_{2}\right) \oplus_{2} M_{3} \ldots \ldots \ldots \ldots \ldots \ldots \ldots$

3.5 A 3-connected matroid union. . . . . . . . . . . . . 44

3.6 Intersection elements in one component. . . . . . . . . . . 45

3.7 One intersection element in each component. . . . . . . . . 46

3.8 When is the union not 3 -connected ? . . . . . . . . 47

4.1 A parallel connection of two Fano matroids. . . . . . . . . . 59

$5.1 E\left(M_{1}\right)$ has 5 elements and $E\left(M_{2}\right)$ has 2 elements. . . . . 66

$5.2 E\left(M_{1}\right)$ has 4 elements and $E\left(M_{2}\right)$ has 3 elements. . . . . 66

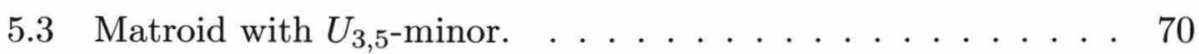


$5.4 x$ and $y$ as parallel pairs. . . . . . . . . . . 75

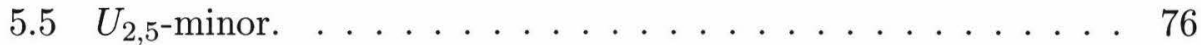

6.1 Minor of a binary matroid that has $x$ and $y$ spilt. . . . . . . 80 


\section{Contents}

Introduction $\quad$ ix

1 Matroids and matroid operations 1

1.1 Independent sets and circuits . . . . . . . . . . . 1

1.2 Rank. . . . . . . . . . . . . . . 2

1.3 Duality ......................... 3

1.4 Minors . . . . . . . . . . . . . . . . . 3

1.5 Connectivity . . . . . . . . . . . . . 4

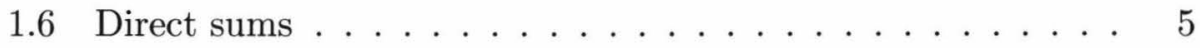

1.7 Representable matroids . . . . . . . . . . . . . . 8

1.8 Series and parallel connections $\ldots \ldots \ldots \ldots$

2 Fundamentals of matroid union $\quad 19$

2.1 Matroid union. . . . . . . . . . . . . . . . 19

2.2 Ground sets of the union . . . . . . . . . . . . . 20

2.3 Properties of matroid union . . . . . . . . . . . . . . 22

2.4 Minors of a decomposable matroid . . . . . . . . . . 27

3 Matroid union and connectivity 33

3.1 The rank of a connected matroid union . . . . . . . . 34

3.2 Matroid union and series connection . . . . . . . . . 35

3.32 -separation in the union . . . . . . . . . . . . . 36

3.4 When is the union 3 -connected ? . . . . . . . . . . . 42 
4 Freedom of elements $\quad 53$

4.1 Fixed elements and clones . . . . . . . . . . . . . . 53

4.2 Freedom of elements in the union . . . . . . . . . . . . . 54

4.3 Freedom of elements in binary and ternary matroids . . . . . 56

4.4 Decomposable binary matroids . . . . . . . . . . . . . . 58

4.4.1 Alternative proof of Cunningham's theorem . . . . . 58

5 Decomposable ternary matroids $\quad 61$

5.1 4-connected ternary matroids are indecomposable . . . . . . . 61

5.2 Property of decomposability . . . . . . . . . . . . . . . 62

5.3 Composing ternary matroids . . . . . . . . . . . . 63

5.4 Decomposing ternary matroids . . . . . . . . . . . . 73

6 What is next? $\quad 79$

6.1 Conjectures . . . . . . . . . . . . . . . . . 79

$\begin{array}{ll}\text { Bibliography } & 81\end{array}$

$\begin{array}{lc}\text { Index } & 82\end{array}$ 


\section{Introduction}

The operation of matroid union was introduced by Nash-Williams in 1966 . A matroid is indecomposable if it cannot be the union of matroids. In 1971 Welsh posed the problem of characterizing indecomposable matroids, this problem has turned out to be extremely difficult. As a partial solution towards its progress, Cunningham characterized binary indecomposable matroids in 1977. In this thesis I present numerous results in topics of matroid union. We start by looking at general properties of matroid union, then proceed to show the link between matroid connectivity and matroid union. Then we look at properties of non-fixed elements in binary and ternary matroids, and how the presence of those elements can inform us about the decomposability of those matroids. Finally we show some results concerning decomposable 3-connected ternary matroids. The concept of fixed and non-fixed elements plays an essential role in proving many results in this thesis.

This thesis has been written with the assumption that the reader is familiar with the basic definitions, ideas and concepts of Matroid Theory. Although Chapter 1 contains all the preliminaries needed, it is only for the purposes of this thesis, and it is far from being an exhaustive reference for matroid theory as a whole. For an exhaustive reference on the basics of matroid theory, the reader is encouraged to read the early sections of [5].

The first section of Chapter 2 introduces the operation of matroid union in a formal way. The second section discusses the important issue of the ground sets of the matroids whose union we are considering. The third section explores some properties of matroid union, and finally we show which minors of a decomposable matroid are also decomposable. 
Chapter 3 discusses the relationship between matroid union and connectivity. First we note why we are not interested in disconnected unions. Then we look at the rank of the connected union, the link with series connections, and what it means to have a 2-separation in a matroid union. The final section answers the question: 'When is the union 3-connected?'.

Chapter 4 investigates properties of fixed and non-fixed elements in the union of two matroids in general, and in binary and ternary matroids in particular. Cunningham's result is restated and reproved in terms of fixed and non-fixed elements, so that an alternative picture of decomposable binary matroids is presented.

Chapter 5 contains results relating to decomposable 3 -connected ternary matroids, including how to compose a ternary matroid. Finally, Chapter 6 presents some conjectures that may be catalysts for further research in the problem of matroid union.

A final note on which results in this thesis are original and which are known. Chapter 1 contains the preliminary information needed for this thesis, so everything in this chapter is known. In Chapter 2, everything prior to Section 2.4 is known, the results of Section 2.4 are not found in the literature, however, they are straightforward. The material of Sections 3.1 and 3.2 of Chapter 3 is known, and the remaining sections of this chapter contain new material. The definitions and results of Section 4.1 of Chapter 4 are known. Section 4.2 contains new material. Theorem 4.3.3 and Corollary 4.3.5 of Section 4.3 are new, and although Corollary 4.3.2 is straightforward, it is not currently present in the literature. The rest of the material in this section is known. The material in Section 4.4 is known but is restated and re-proven using the new material in Section 4.2, and using Lemma 4.3.1 which is known. The results of Sections 5.1, 5.2 and 5.3 in Chapter 5 are new, apart from Proposition 5.3.6 and Theorem 5.3.8. Propositions 5.4.2 - 5.4.4 and Theorem 5.4.5 of Section 5.4 are known, and the rest of the material in the section is new. 


\section{Chapter 1}

\section{Matroids and matroid operations}

This chapter catalogues some basic facts of matroid theory that will be needed later on in this thesis.

\subsection{Independent sets and circuits}

A matroid $M$ is an ordered pair $(E, \mathcal{I})$ consisting of a finite set $E$ and a collection $\mathcal{I}$ of subsets of $E$ satisfying the following three conditions:

(I1) $\emptyset \in \mathcal{I}$.

(I2) If $I \in \mathcal{I}$ and $I^{\prime} \subseteq I$, then $I^{\prime} \in \mathcal{I}$.

(I3) If $I_{1}$ and $I_{2}$ are in $\mathcal{I}$ and $\left|I_{1}\right|<\left|I_{2}\right|$, then there is an element $e$ of $I_{2}-I_{1}$ such that $I_{1} \cup e \in \mathcal{I}$.

Condition (I3) is called the independence augmentation axiom.

If $M$ is the matroid $(E, \mathcal{I})$, then $M$ is a matroid on $E$. The members of $\mathcal{I}$ are the independent sets of $M$, and $E$ is the ground set of $M$. A subset of $E$ that is not in $\mathcal{I}$ is a dependent set. A basis of $M$ is a maximal independent set.

A circuit of an arbitrary matroid $M$ is a minimal dependent set all of 
whose proper subsets are independent, and we shall denote the set of circuits of $M$ by $\mathcal{C}$ or $\mathcal{C}(M)$. Circuits have the following properties:

(C1) $\emptyset \notin \mathcal{C}$.

(C2) If $C_{1}$ and $C_{2}$ are members of $\mathcal{C}$ and $C_{1} \subseteq C_{2}$, then $C_{1}=C_{2}$.

(C3) If $C_{1}$ and $C_{2}$ are distinct members of $\mathcal{C}$ and $e$ in $C_{1} \cap C_{2}$, then there is a member $C_{3}$ of $\mathcal{C}$ such that $C_{3} \subseteq\left(C_{1} \cup C_{2}\right)-e$.

Condition (C3) is the circuit elimination axiom. Next we state some facts about matroids, but first we recall what it means for a matroid to be representable over a field $F$.

The two fundamental classes of matroids arise from matrices and from graphs. A vector matroid is the matroid that arises from a matrix, and a graphic matroid is the matroid that arises from a graph. If $M$ is isomorphic to the vector matroid of a matrix $D$ over a field $F$, then $M$ is said to be representable over $F$ or $F$-representable.

\subsection{Rank}

In this section we define what a rank of a matroid is, and state a fact about ranks. First we define a fundamental matroid construction. Let $M$ be the matroid $(E, \mathcal{I})$ and suppose that $X \subseteq E$. Let $\mathcal{I} \mid X$ be $\{I \subseteq X: I \in \mathcal{I}\}$. Then the pair $(X, \mathcal{I})$ is a matroid. This matroid is the restriction of $M$ to $X$ or the deletion of $E-X$ from $M$. It is denoted by $M \mid X$ or $M \backslash(E-X)$. The rank $r(X)$ of $X$ is the size of a basis $B$ of $M \mid X$. Evidently $r$ maps $2^{E}$ into the set of non-negative integers. This function, the rank function of $M$, will be written as $r_{M}$ or $r$.

Lemma 1.2.1. [5, Lemma 1.3.1] The rank function $r$ of a matroid $M$ on $a$ set $E$ satisfies the following condition:

(R3) If $X$ and $Y$ are subsets of $E$, then

$$
r(X \cup Y)+r(X \cap Y) \leq r(X)+r(Y) .
$$




\subsection{Duality}

In this section we define the dual of a matroid and state a useful property. Note that $\mathcal{B}(M)$ is the set of bases of a matroid $M$.

Theorem 1.3.1. [5, Theorem 2.1.1] Let $M$ be a matroid and $B^{*}(M)$ be $\{E(M)-B: B \in \mathcal{B}(M)\}$. Then $B^{*}(M)$ is the set of bases of a matroid on $E(M)$.

The matroid obtained in the theorem above, whose ground set is $E(M)$, and whose set of bases is $B^{*}(M)$ is the dual of $M$, and is denoted by $M^{*}$. The circuits, hyperplanes, independent sets, and spanning sets of $M$ are the cocircuits, cohyperplanes, coindependent sets, and cospanning sets of $M^{*}$ respectively.

Proposition 1.3.2. [5, Proposition 2.1.6] Let $M$ be a matroid on a set $E$ and suppose $X \subseteq E$. Then

(i) $X$ is independent if and only if $E-X$ is cospanning.

(ii) $X$ is spanning if and only if $E-X$ is coindependent.

(iii) $X$ is a hyperplane if and only if $E-X$ is a cocircuit.

(iv) $X$ is a circuit if and only if $E-X$ is a cohyperplane.

\subsection{Minors}

In this section we define what minors are. If $M$ is a matroid on $E$ and $T$ is a subset of $E$, then the contraction of $T$ from $M$ is denoted $M / T$ and is given by $M / T=\left(M^{*} \backslash T\right)^{*}$. A sequence of deletions and contractions from $M$ can be written in the form $M \backslash X / Y$ for some pair of disjoint sets $X$ and $Y$, where $X, Y \subseteq E(M)$, either of which may be empty. Matroids of this form are minors of $M$.

Proposition 1.4.1. [5, Corollary 3.1.8] Suppose that $B_{T}$ is a basis of $M \mid T$. Then

$$
\begin{aligned}
\mathcal{I}(M / T) & =\left\{I \subseteq E-T: I \cup B_{T} \in \mathcal{I}(M)\right\} \\
& =\{I \subseteq E-T: M \mid T \text { has a basis B such that } B \cup I \in \mathcal{I}(M)\} .
\end{aligned}
$$


Proposition 1.4.2. [5, Proposition 3.1.26] Let $T_{1}$ and $T_{2}$ be disjoint subsets of $E(M)$. Then

(i) $\left(M \backslash T_{1}\right) \backslash T_{2}=M \backslash\left(T_{1} \cup T_{2}\right)=\left(M \backslash T_{2}\right) \backslash T_{1}$;

(ii) $\left(M / T_{1}\right) / T_{2}=M /\left(T_{1} \cup T_{2}\right)=\left(M / T_{2}\right) / T_{1}$; and

(iii) $\left(M \backslash T_{1}\right) / T_{2}=\left(M / T_{2}\right) \backslash T_{1}$.

\subsection{Connectivity}

In this section we define connectivity for matroids and state some important facts about connectivity.

Proposition 1.5.1. [5, Proposition 4.1.4] The matroid $M$ is connected if and only if, for every pair of distinct elements of $E(M)$, there is a circuit containing both.

The following observation follows from the above.

Proposition 1.5.2. A connected matroid with at least two elements does not have any coloops.

Proof. Suppose $M$ is a connected matroid that has a coloop e. By Proposition 1.5.1, there must be a circuit containing $e$ and any other element $f$ of $E(M)$. But since a coloop is not contained in any circuit, then $e$ is not contained in any circuit, giving a contradiction. Therefore a connected matroid does not have any coloops.

Similarly, the following is true for connected matroids.

Proposition 1.5.3. A connected matroid with at least two elements does not have any loops.

Proof. Suppose $M$ is a connected matroid that has a loop $e$. By Proposition 1.5.1, there must be a circuit containing $e$ and any other element $f$ of $E(M)$. But a loop is a 1-element circuit, and therefore is not contained in any circuit other than itself. Hence $e$ is not contained in any circuit other than itself, giving a contradiction. Therefore a connected matroid does not have any loops. 
The following proposition links the connectivity of a matroid with its dual.

Proposition 1.5.4. [5, Corollary 4.2.8] $M$ is connected if and only if $M^{*}$ is connected.

The following proposition describes the structure of a disconnected matroid.

Proposition 1.5.5. [5, Proposition 4.2.11] The matroid $M$ is disconnected if and only if, for some proper non-empty subset $T$ of $E(M), \mathcal{I}(M)=\left\{I_{1} \cup\right.$ $\left.\left.I_{2}: I_{1} \in \mathcal{I}(M \mid T), I_{2} \in \mathcal{I}(M \mid E-T)\right)\right\}$.

\subsection{Direct sums}

In this section we define and state facts about direct sums. Note the following.

Proposition 1.6.1. [5, Proposition 4.2.12] Let $M_{1}$ and $M_{2}$ be matroids on disjoint sets $E_{1}$ and $E_{2}$. Let $E=E_{1} \cup E_{2}$ and $\mathcal{I}=\left\{I_{1} \in \mathcal{I}\left(M_{1}\right), I_{2} \in\right.$ $\left.\mathcal{I}\left(M_{2}\right)\right\}$. Then $(E, \mathcal{I})$ is a matroid.

The matroid $(E, \mathcal{I})$ in the proposition above is the direct sum or 1-sum of $M_{1}$ and $M_{2}$ and is denoted $M_{1} \oplus M_{2}$.

What follows are equivalent ways of describing direct sums.

Proposition 1.6.2. [5, Proposition 4.2.16] $C$ is a circuit of $M_{1} \oplus M_{2}$ if and only if $C$ is either a circuit of $M_{1}$ or a circuit of $M_{2}$.

Proof. Let $C$ be a circuit of $M_{1} \oplus M_{2}$, then we will show that $C$ is either in $\mathcal{C}\left(M_{1}\right)$ or in $\mathcal{C}\left(M_{2}\right)$. If for all $x \in C, x$ was in $E\left(M_{1}\right)$ then $C$ is in $\mathcal{C}\left(M_{1}\right)$, and if for all $x \in C, x$ was in $E\left(M_{2}\right)$ then $C$ is in $\mathcal{C}\left(M_{2}\right)$ and we are done. So suppose the elements of $C$ are from $E\left(M_{1}\right)$ and from $E\left(M_{2}\right)$. Without loss of generality, choose $x \in C$, such that $x$ is in $E\left(M_{1}\right)$. Then $C-\{x\}=I$ is independent in $M_{1} \oplus M_{2}$, and hence of the form $I_{1} \cup I_{2}$ where $I_{1}$ is independent in $M_{1}$ and $I_{2}$ is independent in $M_{2}$. It is also true that for any other element $y \in C$ where $y$ is in $E\left(M_{2}\right), C-\{y\}$ is independent. But $C-\{y\}$ contains $x$ and contains $I_{1}$. Since $x$ is a proper subset of $C$, then 
it is an independent set of $M_{1}$. Meaning $I^{\prime}=I_{1} \cup\{x\}$ is independent in $M_{1}$, because $I^{\prime}$ is a proper subset of $C$. So that $I^{\prime} \cup I_{2}$ must be independent in $M_{1} \oplus M_{2}$, but $I^{\prime} \cup I_{2}=C$, contradiction. Therefore if $C$ is a circuit of $M_{1} \oplus M_{2}$, then it is either in $\mathcal{C}\left(M_{1}\right)$ or in $\mathcal{C}\left(M_{2}\right)$.

Conversely, if $C$ is either a circuit of $M_{1}$ or $M_{2}$, then its elements are either completely from $E\left(M_{1}\right)$ or completely from $E\left(M_{2}\right)$ respectively. Suppose without loss of generality that all its elements are from $E\left(M_{1}\right)$. Consider $C$ in $M_{1} \oplus M_{2}$. $C$ is either dependent or independent in $M_{1} \oplus M_{2}$. It cannot, however, be independent because then it has to be independent in $M_{1}$ when of course it is not. So it must be dependent in $M_{1} \oplus M_{2}$. Moreover, every proper subset of $C$ is independent in $M_{1}$, because $C$ is a circuit of $M_{1}$. It follows that every proper subset of $C$ is independent in $M_{1} \oplus M_{2}$ as well. Therefore $C$ is a minimal dependent set in $M_{1} \oplus M_{2}$, that is a circuit of $M_{1} \oplus M_{2}$.

The rank of a set in the direct sum is calculated as follows.

Proposition 1.6.3. [5, Proposition 4.2.17] If $X \subseteq E\left(M_{1} \oplus M_{2}\right)$ then $r_{M_{1} \oplus M_{2}}(X)=r_{M_{1}}\left(X \cap E\left(M_{1}\right)\right)+r_{M_{2}}\left(X \cap E\left(M_{2}\right)\right)$.

Proof. Let $Y$ be the maximal independent subset of $X$. Then $Y$ can be partitioned into the two independent sets $Y_{1} \subseteq E\left(M_{1}\right)$ and $Y_{2} \subseteq E\left(M_{2}\right)$. But in order for $Y$ to be a maximal independent set, both $Y_{1}$ and $Y_{2}$ have to be maximal independent sets in $M_{1}$ and $M_{2}$ respectively. It follows that $\left|Y_{1}\right|=$ $r_{M_{1}}\left(X \cap E\left(M_{1}\right)\right)$, and $\left|Y_{2}\right|=r_{M_{2}}\left(X \cap E\left(M_{2}\right)\right)$. Therefore $r_{M_{1} \oplus M_{2}}(X)=$ $|Y|=\left|Y_{1}\right|+\left|Y_{2}\right|=r_{M_{1}}\left(X \cap E\left(M_{1}\right)\right)+r_{M_{2}}\left(X \cap E\left(M_{2}\right)\right)$.

Next we look at the bases in the direct sum.

Proposition 1.6.4. [5, Proposition 4.2.18] The set of bases of $M_{1} \oplus M_{2}$ are $\left\{B_{1} \cup B_{2}: B_{1} \in \mathcal{B}\left(M_{1}\right), B_{2} \in \mathcal{B}\left(M_{2}\right)\right\}$.

Proof. Let $B$ be a basis of $M_{1} \oplus M_{2}$. B is a maximal independent set. So it follows from Proposition 1.6.1, that $B$ is the union of two independent sets $I_{1}$ and $I_{2}$, where $I_{1}$ is independent in $M_{1}$ and $I_{2}$ independent in $M_{2}$. But in order for $B$ to be a maximal independent set, both $I_{1}$ and $I_{2}$ have to be maximal independent sets in $M_{1}$ and $M_{2}$ respectively. So that $I_{1}$ and $I_{2}$ are bases of $M_{1}$ and $M_{2}$ respectively. 
The flats of the direct sum are as follows.

Proposition 1.6.5. [5, Proposition 4.2.20] $F$ is a flat of $M_{1} \oplus M_{2}$ if and only if $F \cap E\left(M_{1}\right)$ and $F \cap E\left(M_{2}\right)$ are flats of $M_{1}$ and $M_{2}$ respectively.

Proof. Let $F_{M_{1} \oplus M_{2}}$ denote a flat of $M_{1} \oplus M_{2}$. If $F$ is a flat of $M_{1} \oplus M_{2}$, then $\operatorname{cl}(F)=F$. Let $F=F_{1} \cup F_{2}$, where $F_{1}$ and $F_{1}$ are disjoint sets such that $F_{1}=F \cap E\left(M_{1}\right)$, and $F_{2}=F \cap E\left(M_{2}\right)$. Then $c l(F)=F=F_{1} \cup F_{2}$. By Proposition 1.6.3, any increase in the rank of $F_{1}$, will increase the rank of $F_{M_{1} \oplus M_{2}}$, and any increase in the rank of $F_{2}$ will increase the rank of $F_{M_{1} \oplus M_{2}}$. So it follows that $c l(F)=c_{M_{1}}\left(F_{1}\right) \cup c l_{M_{2}}\left(F_{2}\right)$. So now we have $c l(F)=F=F_{1} \cup F_{2}=c l_{M_{1}}\left(F_{1}\right) \cup c l_{M_{2}}\left(F_{2}\right)$. Since $c l_{M_{1}}\left(F_{1}\right)$ only contains elements from $E\left(M_{1}\right)$, then $c l_{M_{1}}\left(F_{1}\right)$ must be equal to $F_{1}$. Similarly $c l_{M_{2}}\left(F_{2}\right)$ must be equal to $F_{2}$. Therefore $F \cap E\left(M_{1}\right)$ and $F \cap E\left(M_{2}\right)$ are flats of $M_{1}$ and $M_{2}$ respectively.

Conversely, suppose $F \cap E\left(M_{1}\right)$ is a flat of $M_{1}$ and $F \cap E\left(M_{2}\right)$ is a flat of $M_{2}$. Let $F_{1}=F \cap E\left(M_{1}\right)$ and $F_{2}=F \cap E\left(M_{2}\right)$, and let $F=F_{1} \cup F_{2}$. Since $F_{1}$ and $F_{2}$ are flats of $M_{1}$ and $M_{2}$ respectively, then $c_{M_{1}}\left(F_{1}\right)=F_{1}$ and $c l_{M_{2}}\left(F_{2}\right)=F_{2}$. So $F_{1} \cup F_{2}=F=c l_{M_{1}}\left(F_{1}\right) \cup c l_{M_{2}}\left(F_{2}\right)$. But the elements that you can add to $F_{1}$ without increasing its rank in $M_{1}$, together with the elements that you can add to $F_{2}$ without increasing its rank in $M_{2}$, are the elements that you can add to $F$ without increasing its rank in $M_{1} \oplus M_{2}$. In other words $c l_{M_{1}}\left(F_{1}\right) \cup c l_{M_{2}}\left(F_{2}\right)=F=c l(F)$. Therefore $F$ is a flat of $M_{1} \oplus M_{2}$.

The closure operator of the direct sum is as follows.

Proposition 1.6.6. [11, Proposition 7.6.1]. If $X$ is a set in $E\left(M_{1} \oplus M_{2}\right)$, then the closure of $X$ in the direct sum is $c_{M_{1}}\left(X_{1}\right) \cup c_{M_{2}}\left(X_{2}\right)$ for some partition $\left\{X_{1}, X_{2}\right\}$ of $X$, where $X=X_{1} \cup X_{2}, X_{1}=X \cap E\left(M_{1}\right)$, and $X_{2}=$ $X \cap E\left(M_{2}\right)$.

Proof. By Proposition 1.6.3 $r_{M_{1} \oplus M_{2}}(X)=r_{M_{1}}\left(X_{1}\right)+r_{M_{2}}\left(X_{2}\right)$. So $c l(X)=$ $\left\{x: r(X \cup\{x\})=r(X)\right.$ for all $\left.x \in E\left(M_{1} \oplus M_{2}\right)\right\}$. Element $x$ can be either in $E\left(M_{1}\right)$, or in $E\left(M_{2}\right)$. So $c l(X)=\left\{x_{1}: r\left(X_{1} \cup\left\{x_{1}\right\}\right)=r\left(X_{1}\right)\right.$ for $\left.x_{1} \in E\left(M_{1}\right)\right\} \cup\left\{x_{2}: r\left(X_{2} \cup\left\{x_{2}\right\}\right)=r\left(X_{2}\right)\right.$ for $\left.x_{2} \in E\left(M_{2}\right)\right\}$. The aforementioned is equal to $c_{M_{1}}\left(X_{1}\right) \cup c l_{M_{2}}\left(X_{2}\right)$. 


\subsection{Representable matroids}

In this section we state some facts about representable matroids. Recall from Section 1.1 that an $F$-representable matroid, is a matroid isomorphic to the vector matroid of a matrix $D$ over the field $F$. A matroid is binary if it is representable over $G F(2)$, a matroid is ternary if it is representable over $G F(3)$, a matroid is regular if it is both binary and ternary.

Proposition 1.7.1. [5, Corollary 2.2.9] If $M$ is representable over the field $F$, then $M^{*}$ is also representable over $F$.

Proposition 1.7.2. [5, Proposition 3.2.4] Every minor of an F-representable matroid is F-representable.

The next result lists some classes of matroids that are closed under direct sums. Please note that transversal matroids will be defined later.

Proposition 1.7.3. [5, Proposition 4.2.15] The classes of F-representable, graphic, cographic, transversal, and regular matroids are all closed under the operation of direct sum.

One of the important matroids in Matroid Theory, that will be referred to a lot in this thesis is known as the Fano matroid, denoted $F_{7}$ and shown in Figure 1.1 below. The next proposition shows when $F_{7}$ is representable.

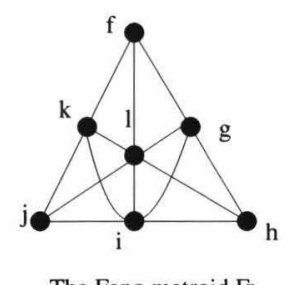

Figure 1.1: The Fano matroid

Theorem 1.7.4. [5, Theorem 6.4.8] Let $F$ be a field. Then $F_{7}$ is Frepresentable if and only if the characteristic of $F$ is two.

The remaining results of this section show what conditions are needed for a matroid to be binary, ternary or regular. 
Theorem 1.7.5. [5, Theorem 6.5.4] A matroid is binary if and only if it has no $U_{2,4}$-minor.

Theorem 1.7.6. [5, Theorem 6.5.7] A matroid is ternary if and only if it has no minor isomorphic to any of the matroids $U_{2,5}, U_{3,5}, F_{7}$ or $F_{7}^{*}$.

Theorem 1.7.7. [5, Theorem 6.6.3] The following statements are equivalent for a matroid $M$ :

(i) $M$ is regular.

(ii) $M$ is representable over every field.

(iii) $M$ is binary and, for some field $F$ of characteristic other than two, $M$ is F-representable.

Theorem 1.7.8. [5, Theorem 6.6.4] A matroid is regular if and only if it has no minor isomorphic to any of the matroids $U_{2,4}, F_{7}$ or $F_{7}^{*}$.

\subsection{Series and parallel connections}

In this section we state some facts about series and parallel connections. We also define and state facts about the 2 -sum operation. We assume the reader is familiar with series connections and parallel connections of matroids, for background information on this subject see [5, Section 7.1].

Let $M_{1}$ and $M_{2}$ be two matroids with $E\left(M_{1}\right) \cap E\left(M_{2}\right)=\{p\}$. Let $E=$ $E\left(M_{1}\right) \cup E\left(M_{2}\right)$. If the collection of circuits of the series connection of $M_{1}$ and $M_{2}$ is $\mathcal{C}_{S}$, and the collection of circuits of the parallel connection of $M_{1}$ and $M_{2}$ is $\mathcal{C}_{P}$, then the following is true:

Proposition 1.8.1. [5, Theorem 7.1.4] Each of $\mathcal{C}_{S}$ and $\mathcal{C}_{P}$ is the collection of circuits of a matroid on $E$.

The matroids on $E$ that have $\mathcal{C}_{S}$ and $\mathcal{C}_{P}$ as their sets of circuits will be denoted by $S\left(M_{1}, M_{2}\right)$ and $P\left(M_{1}, M_{2}\right)$ respectively. In this context, $p$ is the base-point of the connections. What follows are facts about the base-point, series and parallel connections, and the link between series connections and direct sums.

Proposition 1.8.2. [5, Theorem 7.1.8] If the base-point $p$ is a coloop of $M_{1}$, we define $S\left(M_{1}, M_{2}\right)=M_{1} \oplus\left(M_{2} \backslash p\right)$. 
Proposition 1.8.3. [5, Theorem 7.1.11] The base-point $p$ is a coloop of $S\left(M_{1}, M_{2}\right)$ if and only if it is a coloop of $M_{1}$ or $M_{2}$.

Proposition 1.8.4. [5, Theorem 7.1.12] The base-point $p$ is a loop of $P\left(M_{1}, M_{2}\right)$ if and only if it is a loop of $M_{1}$ or $M_{2}$.

Proposition 1.8.5. [5, Proposition 7.1.13] Let $M_{1}$ and $M_{2}$ be matroids with $E\left(M_{1}\right) \cap E\left(M_{2}\right)=\{p\}$. Let $E=E\left(M_{1}\right) \cup E\left(M_{2}\right)$ and $B$ be a subset of $E$.

(i) Assume that, in at least one of $M_{1}$ and $M_{2}, p$ is not a coloop. Then $B$ is a basis of $S\left(M_{1}, M_{2}\right)$ if and only if $B$ can be written as a disjoint union of bases of $M_{1}$ and $M_{2}$.

(ii) Assume that, in at least one of $M_{1}$ and $M_{2}, p$ is not a loop. Then $B$ is a basis of $P\left(M_{1}, M_{2}\right)$ containing $p$ if and only if $B \cap E\left(M_{i}\right)$ is a basis of $M_{i}$ containing $p$ for $i=1$,2. Moreover, $B$ is a basis of $P\left(M_{1}, M_{2}\right)$ not containing $p$ if and only if $p \notin B$ and, for some distinct $i$ and $j$ in $\{1,2\}$, the set $\left[B \cap E\left(M_{i}\right)\right] \cup p$ and $B \cap E\left(M_{j}\right)$ are bases of $M_{i}$ and $M_{j}$, respectively.

Theorem 1.8.6. [5, Theorem 7.1.16] Let $p$ be an element of a connected matroid $M$.

(i) If $M \backslash p=M_{1} \oplus M_{2}$ where both $M_{1}$ and $M_{2}$ are non-empty, then $M=S\left(M / E\left(M_{1}\right), M / E\left(M_{2}\right)\right)$.

Dually,

(ii) If $M / p=M_{1} \oplus M_{2}$ where both $M_{1}$ and $M_{2}$ are non-empty, then $M=$ $P\left(M \backslash E\left(M_{1}\right), M \backslash E\left(M_{2}\right)\right)$.

A matroid is said to have a $k$-separation if there is a partition $(X, Y)$ of the ground set $E$ such that:

(i) $|X|$ and $|Y|$ are each at least $k$, and

(ii) $r(X)+r(Y)-r(E)$ is less than or equal to $k-1$. The smallest $k$ such that $M$ has a $k$-separation is called the connectivity of $M$. If $k$ is at 
least 2, we say that $M$ is $n$-connected for all integers $n$ no larger than $k$. If $k=1$, then we say that $M$ is disconnected. So in general:

(i) If a matroid has $k$-separation then it is not $(k+1)$-connected.

(ii) If a matroid is $k$-connected then it is ( $k$ - 1$)$-connected.

The next example illustrates parts (i) and (ii) of Theorem 1.8.6.

Example 1.8.7. Figures 1.2 and 1.3 illustrate parts (i) and (ii) of Theorem 1.8.6 respectively. In Figure $1.2 M$ is a series connection on base-point $p$. Let $A=\{x, y, z\}, B=\{s, t, u, p\}$. So that $r_{M}(A)+r_{M}(B)=2+3=5=$ $r(M)+1=4+1$, thus indicating that $(A, B)$ is a 2 -separation of $M$. The series connection can be disconnected by deleting the base-point element $p$. So in $M \backslash p$ we get $r_{M \backslash p}(A)+r_{M \backslash p}(B-\{p\})=2+2=4=r(M)$, thus indicating that $(A, B-\{p\})$ is a 1 -separation of $M \backslash p$.

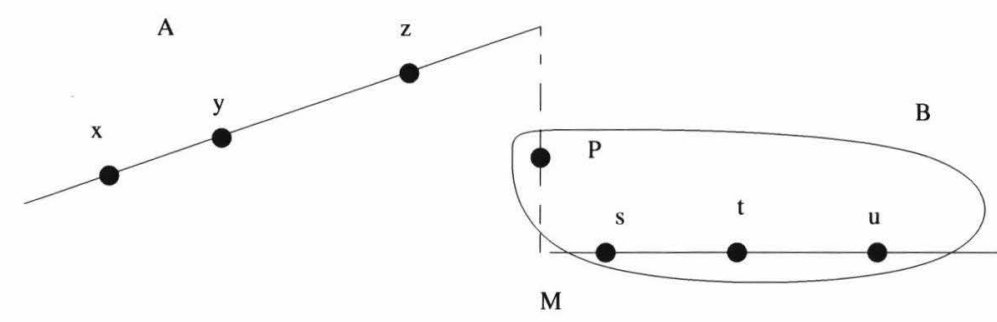

Series connections get disconnected by deleting the basepoint element

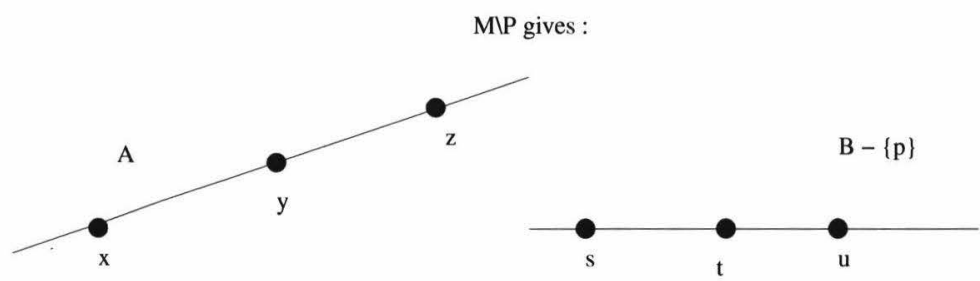

Figure 1.2: Series connection and deletion.

Figure 1.3 shows how a parallel connection can be disconnected by contracting the base-point $p$. Let $A=\{x, y, z\}, B=\{s, t, u, p\}$. So that $r_{M}(A)+r_{M}(B)=2+2=4=r(M)+1=3+1$, thus indicating that $(A, B)$ is a 2-separation of $M$. The parallel connection can be disconnected by contracting the base-point element $p$. So in $M / p$ we get $r_{M / p}(A)+$ $r_{M / p}(B-\{p\})=1+1=2=r(M / p)$, thus indicating that $(A, B-\{p\})$ is 
a 1-separation of $M / p$.

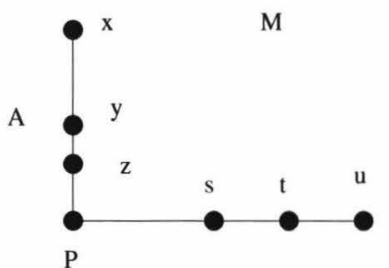

B
If we contract $\mathrm{p}$ we get a 1-separation:

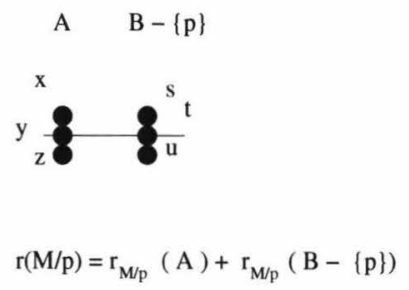

$\mathrm{M}$ is a parallel connection on basepoint $\mathrm{p}$

Figure 1.3: Parallel connection and contraction.

The following proposition shows that the connectivity of the series and parallel connection of two matroids depends on the connectivity of the two matroids.

Proposition 1.8.8. [5, Proposition 7.1.17] If the matroids $M_{1}$ and $M_{2}$ each have at least two elements and $E\left(M_{1}\right) \cap E\left(M_{2}\right)=\{p\}$, then the following statements are equivalent:

(i) Both $M_{1}$ and $M_{2}$ are connected.

(ii) $S\left(M_{1}, M_{2}\right)$ is connected.

(iii) $P\left(M_{1}, M_{2}\right)$ is connected.

Let $M$ and $N$ be matroids each with at least three elements, and let $E(M) \cap E(N)=\{p\}$. Suppose that neither $M$ nor $N$ has $\{p\}$ as a separator. Then the 2-sum of $M$ and $N$ denoted $M \oplus_{2} N$ is $S\left(M_{1}, M_{2}\right) / p$ or, equivalently, $P\left(M_{1}, M_{2}\right) \backslash p$. Clearly $M \oplus_{2} N=N \oplus_{2} M$. $M$ and $N$ are the parts of the 2-sum. What follows are results about 2-sums.

Proposition 1.8.9. [5, Proposition 7.1.19] Both $M$ and $N$ are isomorphic to proper minors of $M \oplus_{2} N$.

Proposition 1.8.10. [5, Proposition 7.1.20] (i) $\left(M \oplus_{2} N\right)^{*}=M^{*} \oplus_{2} N^{*}$. (ii) If $\left|E\left(N_{i}\right)\right| \geq 2$ for $i=1,2$, then $P\left(N_{1}, N_{2}\right) \backslash p$ is connected if and only if both $N_{1}$ and $N_{2}$ are connected. In particular, $M \oplus_{2} N$ is connected if and only if both $M$ and $N$ are connected. 
Some classes of matroids are closed under 2-sums, as shown in the next result.

Proposition 1.8.11. [5, Corollary 7.1.23] The classes of graphic, cographic, F-representable, and regular matroids are all closed under 2-sums.

The following proposition describes the set of circuits in the 2-sum.

Proposition 1.8.12. [6] The 2-sum of $M_{1}$ and $M_{2}$ is the matroid whose ground set is $\left(E_{1} \cup E_{2}\right)-\{p\}$ and whose set of circuits consists of all circuits of $M_{1} \backslash p$ together with all circuits of $M_{2} \backslash p$ and all sets of the form $\left(C_{1} \cup\right.$ $\left.C_{2}\right)-\{p\}$ where each $C_{i}$ is a circuit of $M_{i}$ containing $p$.

The next figure gives a general illustration of the three different ways 2-separation occurs.

Figure 1.4(a) illustrates a 1-separation in $M$. It has a partition $(A, B)$ such that $r(A)+r(B)=r(M)$. Figure 1.4(b), (c) and (d) illustrate the three ways 2 -separation can occur. Part (a) shows a parallel connection, (b) shows a series connection and (c) shows a 2-separation where there is no base-point. Figure 1.4 only gives the general view. The figure in the next example shows concrete instances of 2-separation.

Example 1.8.13. Figure 2.1(a) shows a 1-separation of matroid $M$. The two disjoint sets are $A=\{x\}, B=\{a, b\}$, so that $M=(M \mid A) \oplus(M \mid B)$. Figure 2.1(b) shows a 2-separation as a parallel connection. In (b) $A=$ $\{p, x, y\}$ and $B=\{p, b, c\}$. So that $M=P(M|A, M| B)$. Figure 2.1(c) shows a 2-separation as a series connection. In (c) $A=\{p, x, y, a\}$ and $B=\{p, b, c, d, e\}$. So that $M=S(M|A, M| B)$. Finally Figure $2.1(\mathrm{~d})$ shows a 2 -separation where there is no base-point $p$. Here $A=\{p, a, x, y\}$, and $B=\{p, d, b, c\}$. So that $M=(M \mid A) \oplus_{2}(M \mid B)$.

$F$-representable matroids are also closed under the operation of series and parallel connections, as shown in the next result.

Proposition 1.8.14. [5, Proposition 7.1.21] Let $F$ be a field. If $M_{1}$ and $M_{2}$ are $F$-representable matroids such that $E\left(M_{1}\right) \cap E\left(M_{2}\right)=\{p\}$, then both $P\left(M_{1}, M_{2}\right)$ and $S\left(M_{1}, M_{2}\right)$ are $F$-representable. 


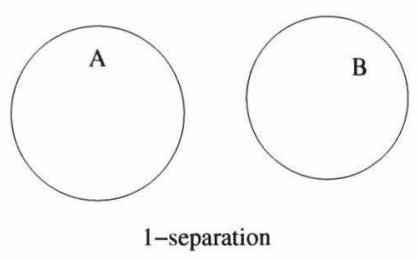

(a)

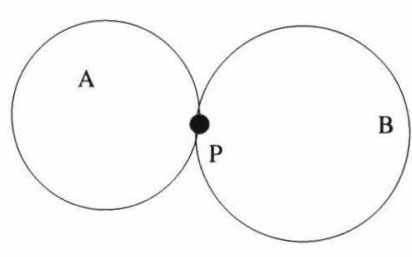

Parallel connection

(b)
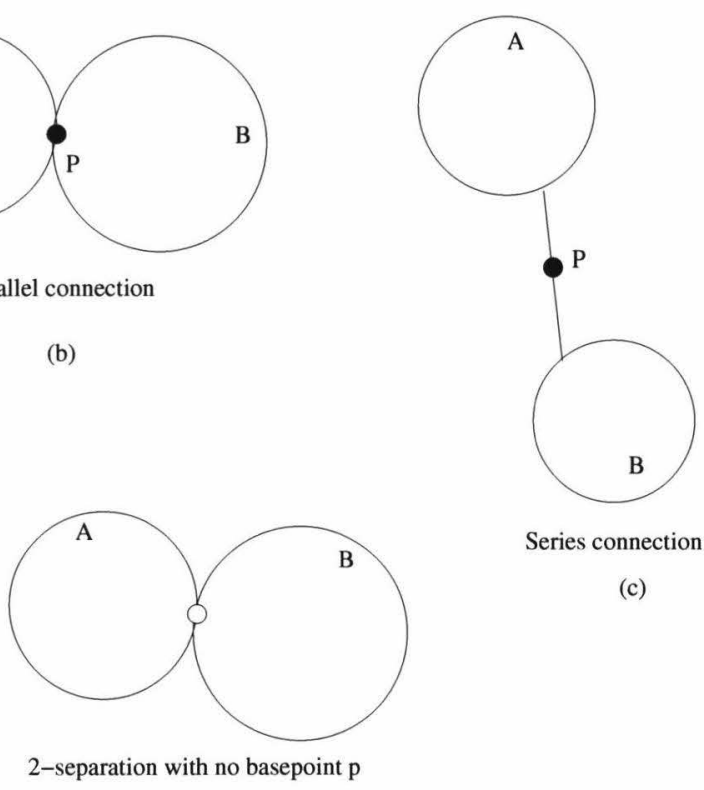

Series connection

(c)

(d)

Figure 1.4: General view of 1-separation and 2-separations. 


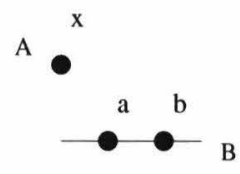

1-separation

(a)

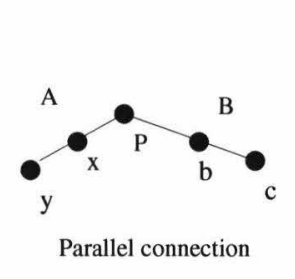

(b)

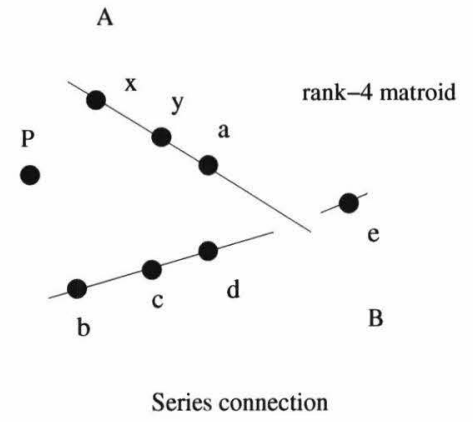

(c)

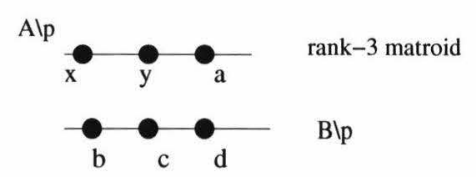

2-separation with no basepoint $p$

(d)

Figure 1.5: Examples of 1- and 2-separations. 
The following proposition ties together the operations of 2-sum, parallel and series connections.

Lemma 1.8.15. Let $M$ be the 2-sum of the two matroids $M_{A}$ and $M_{B}$ where $\{p\}=E\left(M_{A}\right) \cap E\left(M_{B}\right)$ is the base-point. Then the following are equivalent: (i) $M=M_{A} \oplus_{2} M_{B}$.

(ii) $M=P\left(M_{A}, M_{B}\right) \backslash p$.

(iii) $M=S\left(M_{A}, M_{B}\right) / p$.

The operation of 2-sum and 3-connected matroids are linked as will soon be shown in Theorem 1.8.19, but first we state an important fact about connectivity. The next proposition says that given a matroid $M$, if you delete or contract an element from $M$, then the connectivity of $M$ drops by at most one.

Proposition 1.8.16. [5, Proposition 8.1.13] If $e$ is an element of an $n$ connected matroid $M$, then, provided $|E(M)| \geq 2(n-1)$, both $M \backslash e$ and $M / e$ are $(n-1)$-connected.

The connectivity of a matroid $M$, is determined by its connectivity function $r(X)+r(E(M)-X)-r(M)$ defined on all subsets $X$ of $E(M)$.

Lemma 1.8.17. [5, Lemma 8.1.14] A matroid and its dual have the same connectivity function.

The following lemma shows that if the condition $|E(M)| \geq 2(n-1)$ does not hold in Proposition 1.8.16, then the proposition fails.

Lemma 1.8.18. [2, Section 5] Let e be an element of a 3-connected matroid $M$, where $M \cong U_{2,3}$. Then $M \backslash e$ is disconnected.

The following theorem shows the link between 3-connected matroids and 2-sums.

Theorem 1.8.19. [5, Theorem 8.3.1] A 2-connected matroid $M$ is not 3connected if and only if $M=M_{1} \oplus_{2} M_{2}$ for some matroids $M_{1}$ and $M_{2}$, each of which is isomorphic to a proper minor of $M$.

The two following results show how matroids that are not 3 -connected can be constructed. 
Theorem 1.8.20. [5, Theorem 8.3.4] Every matroid that is not 3-connected can be constructed from 3-connected proper minors of it by a sequence of the operations of direct sum and 2-sum.

Proposition 1.8.21. [5, Proposition 8.3.5] Let $M$ be a connected matroid. Then, for some positive integer $k$, there is a collection $M_{1}, M_{2}, \ldots, M_{k}$ of 3-connected matroids and a $k$-vertex tree $T$ with edges labeled $e_{1}, e_{2}, \ldots, e_{k-1}$ and vertices labeled $M_{1}, M_{2}, \ldots, M_{k}$ such that

(i) $E\left(M_{1}\right) \cup E\left(M_{2}\right) \cup \ldots \cup E\left(M_{k}\right)=E(M) \cup\left\{e_{1}, e_{2}, \ldots, e_{k}\right\}$;

(ii) if the edge $e_{i}$ joins the vertices $M_{j 1}$ and $M_{j 2}$, then $E\left(M_{j 1}\right) \cap E\left(M_{j 2}\right)$ is $\left\{e_{i}\right\} ;$ and

(iii) if no edge joins the vertices $M_{j 1}$ and $M_{j 2}$, then $E\left(M_{j 1}\right) \cap E\left(M_{j 2}\right)$ is empty.

Moreover, $M$ is the matroid that labels the single vertex of the tree $T / e_{1}, e_{2}, \ldots, e_{k-1}$ at the conclusion of the following process: contract the edges $e_{1}, e_{2}, \ldots, e_{k-1}$ of $T$ one by one in order; when $e_{i}$ is contracted, its ends are identified and the vertex formed by this identification is labeled by the 2-sum of the matroids that previously labeled the ends of $e_{i}$. 


\section{Chapter 2}

\section{Fundamentals of matroid union}

Given two matroids $M_{1}$ and $M_{2}$, we can consider a way of joining the two matroids, this operation is called 'matroid union'. Here we define it for two matroids, but that can be easily extended for any finite number of matroids.

\subsection{Matroid union}

Let $M_{1}$ and $M_{2}$ be two matroids, and let $\mathcal{I}=\left\{I_{1} \cup I_{2}: I_{1} \in \mathcal{I}\left(M_{1}\right), I_{2} \in\right.$ $\left.\mathcal{I}\left(M_{2}\right)\right\}$. Then the following is true.

Theorem 2.1.1. I is the collection of independent sets of a matroid on $E\left(M_{1}\right) \cup E\left(M_{2}\right)$.

Proof. Consider the set $\mathcal{I}$. Since $\emptyset$ is in both $\mathcal{I}\left(M_{1}\right)$ and $\mathcal{I}\left(M_{2}\right)$ by definition of matroids, and since $\emptyset \cup \emptyset=\emptyset$, then we have $\emptyset \in \mathcal{I}$, and so (I1) is satisfied. Now if $I \in \mathcal{I}$ and $I^{\prime} \subseteq I$, then we have $I=I_{1} \cup I_{2}$ where $I_{1} \in \mathcal{I}\left(M_{1}\right)$ and $I_{2} \in \mathcal{I}\left(M_{2}\right)$. If either $I^{\prime} \subseteq I_{1}$, or $I^{\prime} \subseteq I_{2}$, then $I^{\prime} \in \mathcal{I}\left(M_{1}\right)$, or $I^{\prime} \in$ $\mathcal{I}\left(M_{2}\right)$ respectively. It follows that $I^{\prime} \in \mathcal{I}$. Or else $I^{\prime} \subseteq\left(I_{1} \cup I_{2}\right)$, without being a subset of either $I_{1}$ or $I_{2}$. But in this case $I^{\prime}$ itself is the union of two independent sets, one being the set $I^{\prime} \cap I_{1}=I_{1}^{\prime}$, and the other being $I^{\prime} \cap I_{2}=I_{2}^{\prime}$. That gives $I_{1}^{\prime} \subseteq I_{1}$, implying $I_{1}^{\prime} \in \mathcal{I}\left(M_{1}\right)$, and similarly $I_{2}^{\prime} \subseteq I_{2}$, implying $I_{2}^{\prime} \in \mathcal{I}\left(M_{2}\right)$. Hence $I^{\prime} \in \mathcal{I}$, and (I2) is satisfied. If $X$ and $Y$ are in 
$\mathcal{I}$ with $|X|<|Y|$, then $X=X_{1} \cup X_{2}$, where $X_{1}$ in $\mathcal{I}\left(M_{1}\right)$, and $X_{2}$ in $\mathcal{I}\left(M_{2}\right)$, and similarly $Y=Y_{1} \cup Y_{2}$, where $Y_{1}$ in $\mathcal{I}\left(M_{1}\right)$, and $Y_{2}$ in $\mathcal{I}\left(M_{2}\right)$. So we have $\left|X_{1} \cup X_{2}\right|<\left|Y_{1} \cup Y_{2}\right|$. Without loss of generality suppose $\left|X_{1}\right|<\left|Y_{1}\right|$. Then there exists an element $e$ in $Y_{1}-X_{1}$ such that $\left(X_{1} \cup\{e\}\right) \in \mathcal{I}\left(M_{1}\right)$. Since $X_{2}$ is already in $\mathcal{I}\left(M_{2}\right)$, then $\left(X_{1} \cup X_{2} \cup\{e\}\right) \in \mathcal{I}$. Now that we have (I1), (I2) and (I3) satisfied, we conclude that $\mathcal{I}$ is the collection of independent sets of a matroid on $E\left(M_{1}\right) \cup E\left(M_{2}\right)$.

The matroid constructed via Theorem 2.1.1 is the union of $M_{1}$ and $M_{2}$, and is denoted by $M_{1} \vee M_{2}$. It follows that we can also talk about the operation of decomposition of matroids, where a matroid is written as the union of two matroids. Note that any matroid can be written as the union of itself with a rank-zero matroid. However, since this is a trivial operation, we will not be concerned with it. What we are concerned with is a proper decomposition. A matroid $M$ is decomposable if there exist matroids $M_{1}$ and $M_{2}$ such that $M=M_{1} \vee M_{2}$ where $r\left(M_{1}\right) \geq 1$ and $r\left(M_{2}\right) \geq 1$. Otherwise a matroid is indecomposable. Some authors use the words reducible for decomposable, and irreducible for indecomposable. In this thesis, however, we will always use the words decomposable and indecomposable. The following figure gives an example of a matroid union.

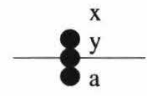

$\mathrm{M}_{1}$

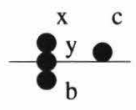

$\mathrm{M}_{2}$

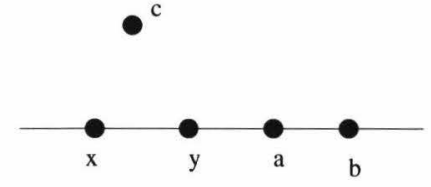

$\mathrm{M}_{1} \mathrm{~V} \mathrm{M}_{2}$

Figure 2.1: An example of a matroid union.

\subsection{Ground sets of the union}

If $M=M_{1} \vee M_{2}$, then it follows from the definition of matroid union that $M_{1}$ and $M_{2}$ may have different ground sets. However, at times it is convenient to have $E\left(M_{1}\right)=E\left(M_{2}\right)$. The next proposition shows that we can always do that. 
Proposition 2.2.1. If a matroid $M$ is decomposable such that $M=M_{1} \vee$ $M_{2}$, then there are matroids $M_{1}^{+}, M_{2}^{+}$on a common ground set $E$ such that $M=M_{1}^{+} \vee M_{2}^{+}$, where $r\left(M_{1}^{+}\right), r\left(M_{2}^{+}\right) \geq 1$.

Proof. If $M_{1}$ and $M_{2}$ above have the same ground set $E$ then we are done. So let us assume that $M_{1}$ and $M_{2}$ do not have a common ground set and that $M_{1}$ is defined over $E_{1}$ and $M_{2}$ defined over $E_{2}$, and let $E=E_{1} \cup E_{2}$. Define $M_{1}^{+}$to be the following extension of $M_{1}$. Take $E_{1}$ and adjoin to it a set of loops where these loops are labeled by the elements of $E-E_{1}$. The same is done for $M_{2}^{+}$. Thus $M_{i}^{+} \mid E_{i}=M_{i}$ and $M_{i}^{+} \mid\left(E-E_{i}\right)$ has rank zero, for $i \in\{1,2\}$. Note that now $M_{1}^{+}$and $M_{2}^{+}$have a common ground set $E$. Since the loops in $M_{1}^{+}$cannot be members of any of the independent sets of $M_{1}^{+}$, then the set of independent sets of $M_{1}^{+}$is the same as the set of independent sets of $M_{1}$. Similarly for $M_{2}^{+}$. So we have $\mathcal{I}\left(M_{1}^{+} \vee M_{2}^{+}\right)=\mathcal{I}\left(M_{1} \vee M_{2}\right)$. Therefore $M_{1}^{+} \vee M_{2}^{+}=M_{1} \vee M_{2}$, with the only difference being that now $M_{1}^{+}$ and $M_{2}^{+}$have common ground sets. So $E\left(M_{1}^{+}\right)=E\left(M_{2}^{+}\right)=E\left(M_{1}^{+} \vee M_{2}^{+}\right)=$ $E(M)$.

The disadvantage of assuming that we have the same ground sets is that $M_{1}$ and $M_{2}$ may have loops that play no structural role. At times it is convenient to assume that $M_{1}$ and $M_{2}$ are loopless. The next proposition shows that we can always do that.

Proposition 2.2.2. If $M$ is a loopless decomposable matroid, then there exist loopless matroids $M_{1}$ and $M_{2}$, each having rank at least one, such that $M=M_{1} \vee M_{2}$.

Proof. Since $M$ is decomposable, then suppose $M=N_{1} \vee N_{2}$. Suppose that $N_{1}$ and $N_{2}$ are not loopless. Consider $e \in E(M)$. If $e \in E\left(N_{1}\right) \cap E\left(N_{2}\right)$ and $e$ is a loop in $N_{1}$ and a loop in $N_{2}$, then $e$ is a loop in $M$ contradicting the fact that $M$ is loopless. So let $A$ be a set of loops of $N_{1}$ without loss of generality. $A$ is a set of loops of $N_{1}$ only by the above. Then $\mathcal{I}\left(N_{1}\right)=\mathcal{I}\left(N_{1} \backslash A\right)$, because $A$ is a set of loops, and therefore none of its elements are contained in any independent set of $N_{1}$. Let $M_{1}=N_{1} \backslash A$. Similarly, let $B$ be the set of loops of $N_{2}$. Let $M_{2}=N_{2} \backslash B$. Then $\mathcal{I}\left(N_{2}\right)=\mathcal{I}\left(N_{2} \backslash B\right)$. It follows that $M=M_{1} \vee M_{2}$, where both $M_{1}$ and $M_{2}$ are loopless. 
In this thesis we will at times assume that $M_{1}$ and $M_{2}$ have different ground sets, and at other times we will assume they have the same ground sets according to convenience. We will always be clear as to which case we are in.

\subsection{Properties of matroid union}

In this section we state some properties of matroid union. We also look at equivalent ways of describing matroid unions when the ground sets are the same. The two following propositions are elementary, they show that matroid union is commutative and associative. In these two propositions we make no assumption about the ground sets.

Proposition 2.3.1. Let $M_{1}$ and $M_{2}$ be two matroids, then $M_{1} \vee M_{2}=$ $M_{2} \vee M_{1}$.

Proposition 2.3.2. Let $M_{1}, M_{2}$ and $M_{3}$ be three matroids, then $\left(M_{1} \vee\right.$ $\left.M_{2}\right) \vee M_{3}=M_{1} \vee\left(M_{2} \vee M_{3}\right)$.

Proof. We observe that a set $I$ in $\left(M_{1} \vee M_{2}\right) \vee M_{3}$ is independent if and only if it is the union of two independent sets $I_{12}$ and $I_{3}$, where $I_{12}$ is independent in $M_{1} \vee M_{2}$, and $I_{3}$ is independent in $M_{3}$. But $I_{12}$ itself must be the union of two independent sets $I_{1}$ and $I_{2}$, where $I_{1}$ is independent in $M_{1}$ and $I_{2}$ independent in $M_{2}$. Since the order in which we take the union of $I_{1}, I_{2}$ and $I_{3}$ does not matter, then it follows that $\left(M_{1} \vee M_{2}\right) \vee M_{3}$ and $M_{1} \vee\left(M_{2} \vee M_{3}\right)$ have exactly the same independent sets. Therefore they are equal.

The following theorem shows what the rank function of the union is when the ground sets are the same.

Theorem 2.3.3. [5, Theorem 12.3.1] Let $M_{1}$ and $M_{2}$ be two matroids on $E$. If $X \subseteq E$, its rank in $M_{1} \vee M_{2}$ is

$$
\min \left\{r_{1}(Y)+r_{2}(Y)+|X-Y|: Y \subseteq X\right\}
$$

where $r_{1}$ and $r_{2}$ are the rank functions of $M_{1}$ and $M_{2}$ respectively. 
To prove Theorem 2.3.3, first we state a couple of results. Note that the notion of matching mentioned in the next theorem will be defined later in case the reader is not already familiar with it.

Theorem 2.3.4. [5, Theorem 12.2.12] Let $\triangle$ be a bipartite graph with vertex classes $S$ and $J$ and let $M$ be a matroid on $J$. Suppose that $\triangle(\mathcal{I})$ is the set of subsets $X$ of $S$ that are matched in $\triangle$ onto a member of $\mathcal{I}(M)$. Then $\triangle(\mathcal{I})$ is the set of independent sets of a matroid $\triangle(M)$ on $S$.

Note that the symbol $\sqcup$ used in the proof for the next proposition indicates a disjoint union.

Proposition 2.3.5. [5, Corollary 12.2.14] If $X \subseteq S$, its rank in $\triangle(M)$ is equal to $\min \left\{r_{M}(N(Y))+|X-Y|: Y \subseteq X\right\}$.

Proof of Theorem 2.3.3. Construct the bipartite graph $\triangle$ as follows. Let $\phi_{1}$ and $\phi_{2}$ be bijections from $E$ onto disjoint sets $E_{1}$ and $E_{2}$. Then for $i=1,2$. the function $\phi_{i}$ induces an isomorphic copy of $M_{i}$ on $E_{i}$. Hence on $E_{1} \sqcup E_{2}$, we have a matroid isomorphic to $M_{1} \oplus M_{2}$. Let $\triangle$ have $E$ as one vertex class and $E_{1} \cup E_{2}$ as the other. Join each element $e$ of $E$ to $\phi_{1}(e)$ and $\phi_{2}(e)$ and to no other vertices in $E_{1} \cup E_{2}$ as in Figure 2.2. Now consider the matroid $N$ on $E$ induced from $M_{1} \oplus M_{2}$ by $\triangle$. Since $M_{1} \oplus M_{2}$ has rank function $r_{1}+r_{2}$, Proposition 2.3.5 implies that the rank function of $N$ is as specified in the theorem. Moreover, one easily checks that $\mathcal{I}(N)=\left\{I_{1} \cup I_{2}: I_{1} \in \mathcal{I}\left(M_{1}\right), I_{2} \in \mathcal{I}\left(M_{2}\right)\right\}$.

When the ground sets are the same, then the collection of circuits in the union is as follows.

Proposition 2.3.6. [11, Proposition 7.6.12] Let $M_{1}$ and $M_{2}$ be two matroids on $E$. The collection of circuits $\mathcal{C}$ in $M_{1} \vee M_{2}$ is as follows: $\mathcal{C}$ consists of minimal sets of the form $\left\{C: A \cap C\right.$ contains a circuit of $M_{1}$, or $C-A$ contains a circuit of $M_{2}$ for all $\left.A \subseteq C\right\}$.

The closure operator for the matroid union $M$, where the ground sets are the same is as follows.

Proposition 2.3.7. [11, Proposition 7.6.12] Let $M_{1}$ and $M_{2}$ be two matroids on $E$. For a set $X$ in $M=M_{1} \vee M_{2}, c l_{M_{1} \vee M_{2}}(X)=X \cup\{x$ : 


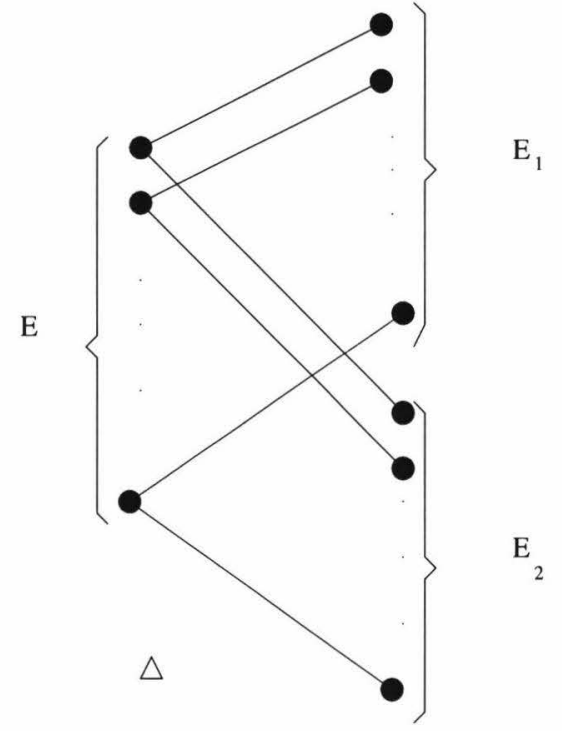

Figure 2.2: $M_{1} \vee M_{2}$ is induced from $M_{1} \oplus M_{2}$ by $\triangle$.

there exists $X_{1}, X_{2} \subseteq X$, with $X_{1} \cap X_{2}=\emptyset$, such that $x \in \operatorname{cl}_{M_{1}}\left(X_{1}\right)$ and $x \in c l_{M_{2}}\left(X_{2}\right), X_{1} \subseteq c l_{M_{2}}\left(X_{2}\right)$, and $\left.X_{2} \subseteq c l_{M_{1}}\left(X_{1}\right)\right\}$.

The bases in the union, when the ground sets are the same are as follows:

Proposition 2.3.8. [11, Proposition 7.6.12] Let $M_{1}$ and $M_{2}$ be two matroids on $E$. $A$ basis $B$ in the union is a maximal set of the form $\left\{B_{1} \cup B_{2}\right.$ : where $B_{1}$ is a basis of $M_{1}$ and $B_{2}$ is a basis of $\left.M_{2}\right\}$.

Proof. Let $B$ be a basis of $M_{1} \vee M_{2} . B$ is a maximal independent set. So it follows from the definition of matroid union, that $B$ is the union of two independent sets $I_{1}$ and $I_{2}$, where $I_{1}$ is independent in $M_{1}$ and $I_{2}$ independent in $M_{2}$. But in order for $B$ to be a maximal independent set, at least one of $I_{1}$ and $I_{2}$ has to be a maximal independent set in $M_{1}$ or $M_{2}$ respectively, say $I_{1}$. The independent set $I_{2}$, however, can always be chosen to be a maximal independent set, even if that requires having common elements between $I_{1}$ and $I_{2}$. So that they are bases of $M_{1}$ and $M_{2}$ respectively.

Having introduced the operation of matroid union, and some properties of the operation, we now state a well known unsolved problem in Matroid Theory. 
Problem 2.3.9. [5, Problem 12.3.9] Characterize all indecomposable matroids.

The problem mentioned above has been solved for some classes of matroids. Some examples of such classes are the classes of graphic, transversal, and binary matroids. The following is a characterization of graphic matroids $M$ which are indecomposable, meaning that the only decomposition possible is the trivial decomposition in which a matroid is the union of itself with the empty matroid.

Theorem 2.3.10. [8] The cycle matroid $M(G)$ of a graph $G$ is indecomposable if and only if the removal of any edge leaves the graph 2-connected.

It is known that if $M(G)$ is the union of two matroids then it is the union of two graphic matroids as well, see [8].

Next we look at a characterization of the decomposition of transversal matroids. If $\mathcal{A}$ is the family $\left(A_{1}, A_{2}, \ldots, A_{m}\right)$ of subsets of a set $S$ and $J=\{1,2, \ldots, m\}$, then the bipartite graph $\triangle[\mathcal{A}]$ associated with $\mathcal{A}$ has vertex set $S \cup J$; its edge set is $\left\{x j: x \in S, j \in J\right.$, and $\left.x \in A_{j}\right\}$. A matching in a graph is a set of edges in the graph no two of which have a common endpoint. A subset $X$ of $S$ is a partial transversal of $\mathcal{A}$ if and only if there is a matching in $\triangle[\mathcal{A}]$ in which every edge has one endpoint in $X$. Note the following.

Theorem 2.3.11. [5, Theorem 1.6.2] Let $\mathcal{A}$ be a family $\left(A_{1}, A_{2}, \ldots, A_{m}\right)$ of subsets of a set $S$. Let $I$ be the set of partial transversals of $\mathcal{A}$. Then $I$ is the collection of independent sets of a matroid on $S$.

The matroid obtained above from the set of partial transversals of $\mathcal{A}$ is denoted by $M[\mathcal{A}]$. If $M$ is an arbitrary matroid and $M \cong M[\mathcal{A}]$ for some family $\mathcal{A}$ of sets, then $M$ is a transversal matroid.

In other words, if $M$ is a transversal matroid, then there is a bipartite graph $G$ with vertex sets $V$ and $E(M)$, such that $I \subseteq E(M)$ is independent in $M$ if and only if $I$ can be matched in $G$. In this case $I$ is called a partial transversal of $V$. For $I$ to be matched means that every element $x$ in $I$ is connected to exactly one unique point in $V$. 
The following construction will be used in the next proposition. Say $V=\{1,2, \ldots, n\}$. For $i$ in $V$, define $M_{i}$ on $E(M)$ as follows: if $e$ is in $E(M)$ and there is an edge from $e$ to $i$ then $\{e\}$ is independent in $M_{i}$, otherwise $e$ is a loop in $M_{i}$. If $\{e, f\} \subseteq E(M)$ and $e$ and $f$ are both connected to $i$ in $V$, then $\{e, f\}$ is dependent in $M_{i} . M_{i}$ in this case is a rank-1 matroid. The following is the characterization of the decomposition of transversal matroids.

Proposition 2.3.12. [5, Proposition 12.3.7] A matroid $M$ with rank at least one is transversal if and only if it is a union of rank-1 matroids.

Proof. Since we are working with a transversal matroid $M$, then we can construct a bipartite graph $G$ as follows. It has vertex sets $V$ and $E(M)$, where $V$ is the set of rank- 1 matroids $M_{1}, M_{2}, \ldots, M_{n}$, and $E(M)$ is the set of elements of $M$. If $I$ is independent in the transversal matroid $M$, then $I$ can be matched in $G$. Meaning that every element in $I$ has exactly one endpoint in $V$. Since each of those single element sets have exactly one endpoint in $V$, then $I$ meets the requirement of being independent in $M_{i}$. Implying that $I$ can be partitioned into single element sets, each of which is a match in $G$. Therefore each element of $I$ is independent in some rank-1 matroid $M_{i}$. But $I$ is the union of those single element independent sets. So $I$ is independent in $M_{1} \vee M_{2} \vee \cdots \vee M_{n}$. Therefore if $I$ is independent in $M$ then $I$ is independent in $M_{1} \vee M_{2} \vee \cdots \vee M_{n}$.

Conversely, if $I$ is independent in $M_{1} \vee M_{2} \vee \cdots \vee M_{n}$, then $I$ is the union of $n$ number of independent sets, each having exactly one element. Meaning each one-element set is a match in $G$. But because each one-element set of $I$ is a match in $G$, then $I$ itself is a match in $G$. So it follows that $I$ is a transversal of $M_{1} \vee M_{2} \vee \cdots \vee M_{n}=M$. But if $I$ is a transversal of $M$, then $I$ is independent in $M$ by definition. Therefore if $I$ is independent in $M_{1} \vee M_{2} \vee \cdots \vee M_{n}$, then $I$ is independent in $M$. The two directions show that a matroid $M$ is transversal if and only if it is the union of rank-1 matroid.

The class of uniform matroids is also another class of decomposable matroids. But first we note the following.

Proposition 2.3.13. All uniform matroids are transversal. 
Proof. Let $\mathcal{A}$ be a family of independent sets of $M$, where $M$ is a uniform matroid. Consider the general form of uniform matroids $U_{m, n}$. All the independent sets in $M$ have size less than or equal to $m$. Since $m \leq n$, then we can construct a bipartite graph $\triangle[\mathcal{A}]$ as follows. It has vertex sets $N$ of $n$ elements and $M$ of $m$ elements. Each edge represents an element in a set, the element is the endpoint of the edge in $M$, and so a certain collection of edges represents a certain set. It follows each and every independent set of $M$ is a matching in $\triangle[\mathcal{A}]$, because $m \leq n$. Meaning that the set $\mathcal{I}$ of independent sets of $M$ are partial transversals of $\mathcal{A}$. Therefore by Theorem 2.3.11, $M$ is a transversal matroid.

By combining Propositions 2.3.12 and 2.3.13 we get:

Corollary 2.3.14. All uniform matroids of rank at least 2 are decomposable.

Another class for which there exists a characterization of decomposability is the class of binary matroids. It has been solved by Cunningham. In Chapter 4 we will give an alternative proof of his result. Here we state Cunningham's original result.

Theorem 2.3.15. [1] $A$ binary matroid $M$ on $E$ is indecomposable if and only if $M$ is connected and $M \backslash e$ is connected for each $e \in E$.

As a corollary of Theorem 2.3.15 we get the following.

Corollary 2.3.16. With the exception of $U_{2,3}$, every 3-connected binary matroid is indecomposable.

Proof. A binary matroid is decomposable by Theorem 2.3.15 if and only if it contains an element whose deletion disconnects the matroid. But by Proposition 1.8.16 a 3-connected matroid cannot be disconnected by deleting one element. The only exception to this case, however, is $U_{2,3}$ as stated in [2, Section 5]. Therefore with the exception of $U_{2,3}, 3$-connected binary matroids are indecomposable.

\subsection{Minors of a decomposable matroid}

In this section we show which minors of a decomposable matroid are also decomposable. First we look at minors obtained by deletions. In the special 
case when the ground sets of $M_{1}$ and $M_{2}$ are the same, the following is true.

Proposition 2.4.1. Let $M$ be a decomposable matroid so that $M=M_{1} \vee$ $M_{2}$, where $E\left(M_{1}\right)=E\left(M_{2}\right)$. If $X \subseteq E(M)$, then

(i) $M \backslash X=\left(M_{1} \backslash X\right) \vee\left(M_{2} \backslash X\right)$.

(ii) If neither $M_{1} \backslash X$ nor $M_{2} \backslash X$ consists entirely of loops, then $M \backslash X$ is decomposable.

Proof. (i) If $I$ is independent in $M \backslash X$, then $I$ is independent in $M$, so that $I=I_{1} \cup I_{2}$, where $I_{1}$ is independent in $M_{1}$, and $I_{2}$ is independent in $M_{2}$. As $I \cap X=\emptyset$, then $I_{1}$ is independent in $M_{1} \backslash X$, and $I_{2}$ is independent in $M_{2} \backslash X$. Therefore $I$ is independent in $\left(M_{1} \backslash X\right) \vee\left(M_{2} \backslash X\right)$. Conversely, if $I$ is independent in $\left(M_{1} \backslash X\right) \vee\left(M_{2} \backslash X\right)$, then $I=I_{1} \cup I_{2}$, where $I_{1}$ is independent in $M_{1} \backslash X$, and $I_{2}$ is independent in $M_{2} \backslash X$. It follows that $I_{1}$ is independent in $M_{1}$, and $I_{2}$ is independent in $M_{2}$. Meaning $I$ is independent in $M$. But $I \cap X=\emptyset$. Therefore $I$ is independent in $M \backslash X$. (ii) By (i) $M \backslash X=\left(M_{1} \backslash X\right) \vee\left(M_{2} \backslash X\right)$. If neither $M_{1} \backslash X$ nor $M_{2} \backslash X$ consists entirely of loops, then $r\left(M_{1} \backslash X\right)>0$ and $r\left(M_{2} \backslash X\right)>0$. So by definition of decomposable matroids in Section 2, $M \backslash X$ is decomposable.

More generally, the following is true.

Proposition 2.4.2. Let $M=M_{1} \vee M_{2}$, and suppose $x \in E(M)$. Then

(i) $M \backslash x=\left(M_{1} \backslash x\right) \vee\left(M_{2} \backslash x\right)$ if $x \in E\left(M_{1}\right) \cap E\left(M_{2}\right)$.

(ii) $M \backslash x=\left(M_{1} \backslash x\right) \vee M_{2}$ if $x \in E\left(M_{1}\right)-E\left(M_{2}\right)$.

Proof. Proof of (i) is the same as that of Proposition 2.4.1(i). Consider (ii). If $I$ is independent in $M \backslash x$, then $I$ is independent in $M$, so that $I=I_{1} \cup I_{2}$, where $I_{1}$ is independent in $M_{1}$, and $I_{2}$ is independent in $M_{2}$. As $I \cap x=\emptyset$, then $I_{1}$ is independent in $M_{1} \backslash x$, and $I_{2}$ is independent in $M_{2}$. Therefore $I$ is independent in $\left(M_{1} \backslash x\right) \vee M_{2}$. Conversely, if $I$ is independent in $\left(M_{1} \backslash x\right) \vee M_{2}$, then $I=I_{1} \cup I_{2}$, where $I_{1}$ is independent in $M_{1} \backslash x$, and $I_{2}$ is independent in $M_{2}$. Meaning $I_{1}$ is independent in $M_{1}$, and $I_{2}$ is independent in $M_{2}$. So that $I$ is independent in $M$. But $I \cap x=\emptyset$. Therefore $I$ is independent in $M \backslash x$.

The following are two immediate corollaries. 
Corollary 2.4.3. If $M=M_{1} \vee M_{2}$, where $D \subseteq E(M)$, then $M \mid D=$ $M_{1}\left|\left(D \cap E\left(M_{1}\right)\right) \vee M_{2}\right|\left(D \cap E\left(M_{2}\right)\right)$.

Corollary 2.4.4. If $M=M_{1} \vee M_{2}$, where $D \subseteq E\left(M_{1}\right)-E\left(M_{2}\right)$, then $M_{1}\left|D=\left(M_{1} \vee M_{2}\right)\right| D$. In particular, if $I \subseteq D$, then $I$ is independent in $M_{1} \vee M_{2}$ if and only if $I$ is independent in $M_{1}$.

Second, we look at minors obtained by contractions.

Proposition 2.4.5. Let $M_{1}$ and $M_{2}$ be two matroids such that $x \in E\left(M_{1}\right)$ $E\left(M_{2}\right)$. Then

(i) $\left(M_{1} \vee M_{2}\right) / x=\left(M_{1} / x\right) \vee M_{2}$.

(ii) If $M_{1} / x$ does not consist entirely of loops, then $M / x$ is decomposable.

Proof. (i) Consider $\left(M_{1} \vee M_{2}\right) / x$. If $x$ is a loop, then $\left(M_{1} \vee M_{2}\right) / x=$ $\left(M_{1} \vee M_{2}\right) \backslash x$ and it follows from Proposition 2.4.2(ii) that $\left(M_{1} \vee M_{2}\right) \backslash x=$ $\left(M_{1} \backslash x\right) \vee M_{2}=\left(M_{1} / x\right) \vee M_{2}$. So assume $x$ is not a loop. If $I$ is independent in $\left(M_{1} \vee M_{2}\right) / x$, then $I \cup\{x\}$ is independent in $M_{1} \vee M_{2}$ by Proposition 1.4.1. Hence $I \cup\{x\}=I_{1} \cup I_{2}$, where $I_{1}$ is independent in $M_{1}$ and $I_{2}$ is independent in $M_{2}$. As $x \notin E\left(M_{2}\right), x \in I_{1}$, so that $I_{1}=I_{1}^{\prime} \cup\{x\}$. Now $I_{1}^{\prime}$ is independent in $M_{1} / x$. Therefore $I=I_{1}^{\prime} \cup I_{2}$ where $I_{1}^{\prime}$ is independent in $M_{1} / x$ and $I_{2}$ independent in $M_{2}$. Therefore $I$ is independent in $\left(M_{1} / x\right) \vee M_{2}$. Conversely, if $I$ is independent in $\left(M_{1} / x\right) \vee M_{2}$, then $I=I_{1} \cup I_{2}$, where $I_{1}$ is independent in $M_{1} / x$ and $I_{2}$ independent in $M_{2}$. So that $I_{1}^{\prime}=I_{1} \cup\{x\}$ is independent in $M_{1}$. It follows that $I \cup\{x\}=I_{1}^{\prime} \cup I_{2}$ is independent in $M_{1} \vee M_{2}$. Therefore $I$ is independent in $\left(M_{1} \vee M_{2}\right) / x$ by Proposition 1.4.1. (ii) By (i) $\left(M_{1} \vee M_{2}\right) / x=\left(M_{1} / x\right) \vee M_{2}$. If $M_{1} / x$ does not consist entirely of loops, then $r\left(M_{1} / x\right)>0$. Therefore by the definition of decomposable matroids in Section 2, $M / x$ is decomposable.

We shall give two examples, the first one shows how $\left(M_{1} \vee M_{2}\right) / x$ is not equal to $\left(M_{1} / x\right) \vee\left(M_{2} / x\right)$ when $x$ is in both $M_{1}$ and $M_{2}$. The second example shows that a decomposable matroid may become indecomposable by contracting an element from $E\left(M_{1}\right) \cap E\left(M_{2}\right)$.

Example 2.4.6. Consider the matroid union shown in Figure 2.3. Here $E\left(M_{1}\right)=\{x, y, a\}, E\left(M_{2}\right)=\{x, y, b\}$ and $E(M)=\{x, y, a, b\} . M_{1}, M_{2} \cong$ 
$U_{1,3}$ and $M \cong U_{2,4}$ as shown in (a). We see that $M_{1} / x \cong U_{0,2}$, and $M_{2} / x \cong$ $U_{0,2}$. Therefore $\left(M_{1} / x\right) \vee\left(M_{2} / x\right) \cong U_{0,3}$. Whereas $M / x \cong U_{1,3}$. Therefore $M / x \neq\left(M_{1} / x\right) \vee\left(M_{2} / x\right)$ as shown in (b).

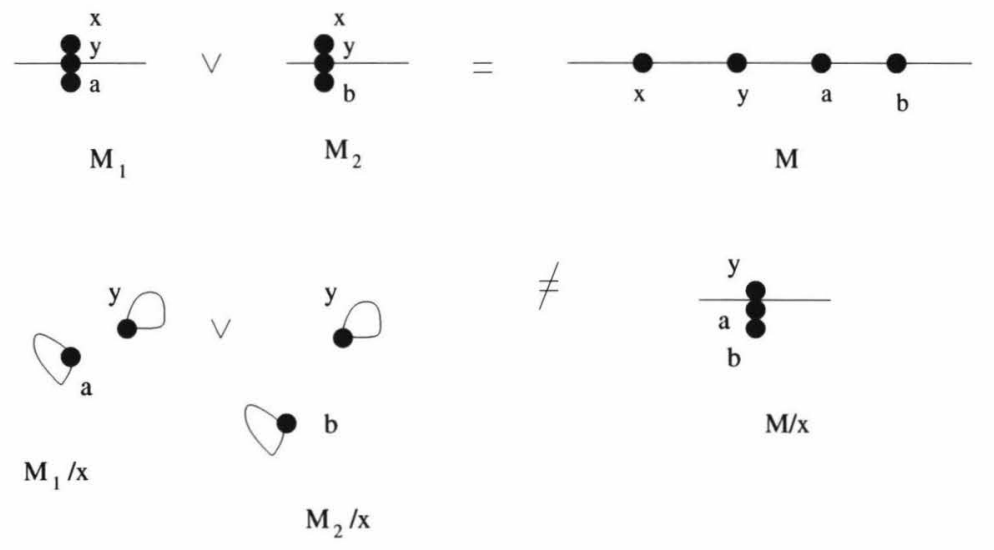

Figure 2.3: Contracting an intersection element.

Example 2.4.7. This example shows that if $x \in E\left(M_{1}\right) \cap E\left(M_{2}\right)$, then $\left(M_{1} \vee M_{2}\right) / x$ is indecomposable. Consider the matroid $M=M_{1} \oplus_{2} M_{2}$ shown in Figure 2.4. Here $E\left(M_{1}\right)=\{a, b, c, d, x, r, p\}, E\left(M_{2}\right)=\{f, g, h, i, q, e, p\}$, where $M_{1}, M_{2} \cong F_{7}$. By Lemma 1.8 .15 (iii) we have $M=S\left(M_{1}, M_{2}\right) / p$. Proposition 3.2 .1 in the next section gives $M_{1} \vee M_{2}=S\left(M_{1}, M_{2}\right)$. So $M=\left(M_{1} \vee M_{2}\right) / p$. But by combining Theorem 3.3.1 with Lemma 4.2.3 that we shall encounter later, we get that $M$ is indecomposable because both $M_{1}$ and $M_{2}$ are indecomposable. 


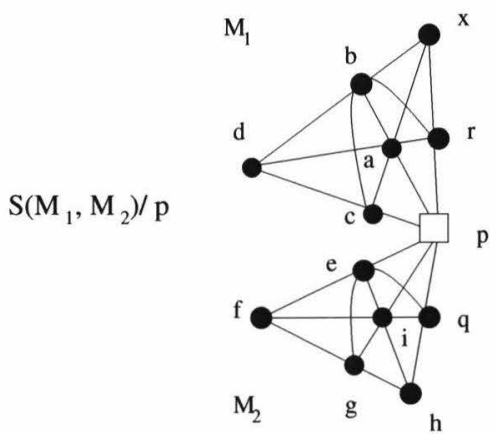

Figure 2.4: Contracting an intersection element may give an indecomposable matroid. 


\section{Chapter 3}

\section{Matroid union and connectivity}

A disconnected matroid $M$ is simply the direct sum of two disjoint matroids by Proposition 1.6.1. That of course is the same as saying $M$ is the union of two disjoint matroids. So the case of decomposing disconnected matroids is trivial. Therefore we shall focus entirely on connected matroids from now on.

Sometimes the connectivity of a matroid can tell us whether the matroid is decomposable or not. Being decomposable does not necessarily mean having a low connectivity. In general it is possible to be decomposable and at the same time be highly connected. An example of this is $U_{100,200}$ which is highly connected but by corollary 2.3 .14 is decomposable. However, if a matroid is the union of two matroids whose ground sets have few elements in common, then that can tell us something about the connectivity of the matroid $M$. In this chapter we explore the connection between matroid union and connectivity. First we look at the rank of the connected union. Second we look at the relationship between matroid union and series connection, then we look at the union that has a 2 -separation, and finally we look at when the union is 3 -connected. 


\subsection{The rank of a connected matroid union}

The following is a Theorem of Cunningham [1, Theorem 2]. First we need a definition. Let $M$ be a matroid and $i$ be a positive integer not exceeding $r(M)$. Then $M^{\prime}$ is a truncation of $M$ denoted $T^{i}(M)$, if $\mathcal{I}\left(T^{i}(M)\right)=\{X \subseteq$ $\mathcal{I}(M):|X| \leq r(M)-i\}$. We state Cunningham's result below for two matroids only.

Theorem 3.1.1. If $M=M_{1} \vee M_{2}$, then there exists a truncation $M_{1}^{\prime}$ for $M_{1}$, and $M_{2}^{\prime}$ for $M_{2}$, such that $r(M)=r\left(M_{1}^{\prime}\right)+r\left(M_{2}^{\prime}\right)$, and we have $M=$ $M_{1}^{\prime} \vee M_{2}^{\prime}$. Moreover, if $M$ has no coloop, then the only choices for $M_{1}^{\prime}$ and $M_{2}^{\prime}$ are $M_{1}$ and $M_{2}$ respectively.

The two following results are corollaries of Theorem 3.1.1, the first one is immediate. The second is for the case when the matroid union is connected.

Corollary 3.1.2. If $M=M_{1} \vee M_{2}$ is a matroid with no coloops, then $r(M)=r\left(M_{1}\right)+r\left(M_{2}\right)$.

Corollary 3.1.3. If $M=M_{1} \vee M_{2}$ is a connected matroid with rank at least two, then $r(M)=r\left(M_{1}\right)+r\left(M_{2}\right)$.

Proof. By Theorem 3.1.1 $r(M)=r\left(M_{1}^{\prime}\right)+r\left(M_{2}^{\prime}\right)$, where $M_{1}^{\prime}$ and $M_{2}^{\prime}$ are truncations of $M_{1}$ and $M_{2}$ respectively. Since $M$ is connected, then by Proposition 1.5.2 $M$ has no coloops. So it follows by Theorem 3.1.1 that the only choices for $M_{1}^{\prime}$ and $M_{2}^{\prime}$ are $M_{1}$ and $M_{2}$ respectively. Therefore $r(M)=r\left(M_{1}\right)+r\left(M_{2}\right)$.

The following is a corollary about the bases in a connected matroid union.

Corollary 3.1.4. If $M=M_{1} \vee M_{2}$ has no coloops, then $B$ is a basis of $M$ if and only if $B=B_{1} \sqcup B_{2}$ where $B_{1}$ and $B_{2}$ are bases of $M_{1}$ and $M_{2}$ respectively.

Proof. If $B$ is a basis of $M$, then by definition of matroid union we have $B=I_{1} \sqcup I_{2}$, where $I_{1}$ is independent in $M_{1}$ and $I_{2}$ independent in $M_{2}$. Since $M$ has no coloops, then by Corollary 3.1.2 $r(M)=r\left(M_{1}\right)+r\left(M_{2}\right)$. 
Let $B_{1}$ be a basis of $M_{1}$, and $B_{2}$ be a basis of $M_{2}$. Since $r(M)=|B|$, $r\left(M_{1}\right)=\left|B_{1}\right|$ and $r\left(M_{2}\right)=\left|B_{2}\right|$, then

$$
|B|=\left|B_{1}\right|+\left|B_{2}\right|
$$

But $|B|=\left|I_{1} \cup I_{2}\right|=\left|I_{1}\right|+\left|I_{2}\right|$, and hence $I_{1}$ must be a basis of $M_{1}$ and $I_{2}$ a basis of $M_{2}$. Conversely, if $B=B_{1} \sqcup B_{2}$, where $B_{1}$ and $B_{2}$ are bases of $M_{1}$ and $M_{2}$ respectively, then $\left|B_{1}\right|=r\left(M_{1}\right)$, and $\left|B_{2}\right|=r\left(M_{2}\right)$. So

$$
|B|=\left|B_{1} \cup B_{2}\right|=\left|B_{1}\right|+\left|B_{2}\right|=r\left(M_{1}\right)+r\left(M_{2}\right) .
$$

But $r\left(M_{1}\right)+r\left(M_{2}\right)=r(M)$ by Corollary 3.1.2. So that $r(M)=|B|$, hence $B$ is a basis of $M$.

\subsection{Matroid union and series connection}

In this section we look at the relationship between matroid union and series connection. We prove the following proposition, which is left as an exercise in [5].

Proposition 3.2.1. [5, Proposition 12.3.6] Let $M_{1}$ and $M_{2}$ be two matroids. If $E\left(M_{1}\right) \cap E\left(M_{2}\right)=\{p\}$, then $M_{1} \vee M_{2}=S\left(M_{1}, M_{2}\right)$.

First we observe the following.

Lemma 3.2.2. Let $M_{1}$ and $M_{2}$ be matroids and assume that $z$ is a coloop of $M_{1}$ or $M_{2}$. Then $z$ is a coloop of $M_{1} \vee M_{2}$.

Proof. Assume without loss of generality that $z$ is a coloop of $M_{1}$, and assume that $B$ is a basis of $M_{1} \vee M_{2}$. Then $B=B_{1} \cup B_{2}$, where $B_{1}$ is a basis of $M_{1}$, and $B_{2}$ is a basis of $M_{2}$. If $z \notin B$, then $z \notin B_{1}$. But as $z$ is a coloop of $M_{1}$, it follows that $z$ should be in every basis of $M_{1}$, so we have a contradiction. Therefore $z$ is in every basis of $M$ meaning $z$ is a coloop of $M$.

Proof of Proposition 3.2.1. There are two cases to consider.

Case 1. $p$ is not a coloop in at least one of $M_{1}$ and $M_{2}$. By Proposition 1.8.5, $B$ is a basis of $S\left(M_{1}, M_{2}\right)$ if and only if $B=B_{1} \sqcup B_{2}$, where $B_{1}$ is a 
basis of $M_{1}$ and $B_{2}$ is a basis of $M_{2}$. It follows from the definition of matroid union that $B$ is a basis of $M_{1} \vee M_{2}$. Therefore $S\left(M_{1}, M_{2}\right)=M_{1} \vee M_{2}$.

Case 2. $p$ is a coloop of both $M_{1}$ and $M_{2}$. By Lemma 3.2.2, $p$ is a coloop of $M_{1} \vee M_{2}$. So that, without loss of generality, $M_{1} \vee M_{2}=\left(M_{1} \backslash p\right) \oplus$ $\left(M_{2} \backslash p\right) \oplus\left(M_{2} \mid\{p\}\right)$. But $\left(M_{2} \backslash p\right) \oplus\left(M_{2} \mid\{p\}\right)=M_{2}$. So that

$$
M_{1} \vee M_{2}=\left(M_{1} \backslash p\right) \oplus M_{2}
$$

By Proposition 1.8.2, 3.2.1 is equal to $S\left(M_{1}, M_{2}\right)$.

\section{$3.3 \quad 2$-separation in the union}

In this section we look at the connected matroid union that has a 2-separation. The following theorem is the main result of this section.

Theorem 3.3.1. Let $(A, B)$ be a 2-separation of $M$ such that $M=M_{A} \oplus_{2}$ $M_{B}$. If $M$ is decomposable, then at least one of $M_{A}$ and $M_{B}$ is decomposable.

The next few results will be used in constructing a proof for the theorem above.

Proposition 3.3.2. Let $M$ be a connected matroid, then the following is true:

(i) If $M \backslash x$ is not connected, where $x$ is in $E(M)$, then $M$ is decomposable.

(ii) If $M$ has a series pair then $M$ is decomposable.

Proof. (i) If $M \backslash x$ is disconnected, then it is a series connection by Theorem 1.8.6(i), and then it follows by Proposition 3.2.1 that it is a union of two matroids. (ii) If $M$ has a series pair $\{a, b\}$, then $M \backslash a$ and $M \backslash b$ have coloops, which implies they are both disconnected by Proposition 1.5.2. From part(i) now of the proposition it follows that $M$ is decomposable.

Lemma 3.3.3. Let $(A, B)$ be a 2-separation of a connected matroid $M$ where $M=M_{A} \oplus_{2} M_{B}$ and $p$ is the base-point. If $c$ is a coloop of $M \mid A$, then $\{c, p\}$ is a series pair of $M_{A}$, similarly, if $c$ is a coloop of $M \mid B$, then $\{c, p\}$ is a series pair of $M_{B}$. 
Proof. By a property of 2-sum, $M_{A} \backslash p=M \mid A$. It follows that $c$ is a coloop of $M_{A} \backslash p$. So that $A-c$ is a hyperplane of $M_{A} \backslash p$ by Proposition 1.3.2(iii). If $p \in c l_{M_{A}}(A-c)$, then $c$ is a coloop of $M_{A}$, implying that $M_{A}$ is disconnected by Proposition 1.5.2. But because $M$ is connected, then $M_{A}$ must also be connected by Proposition 1.8.10(ii), giving a contradiction. So $p \notin c l_{M_{A}}(A-c)$, meaning $A-c$ is a hyperplane of $M_{A}$. Hence $\{p, c\}$ is a cocircuit of $M_{A}$, that is a series pair of $M_{A}$.

Lemma 3.3.4. If $M \mid A$ has a coloop, then $M_{A}$ is decomposable.

Proof. If $M \mid A$ has a coloop, then by Lemma 3.3 .3 above $M_{A}$ has a series pair. But if $M_{A}$ has a series pair, then it follows by Proposition 3.3.2(ii) that $M_{A}$ is decomposable.

Finally before constructing a proof for Theorem 3.3.1, we need a definition. If a matroid $M$ is obtained from a matroid $N$ by deleting a non-empty subset $T$ of $E(N)$, then $N$ is an extension of $M$. In particular, if $|T|=1$, then $N$ is a single-element extension of $M$. Two obvious ways to extend a matroid $M$ are to adjoin a loop or to adjoin a coloop. In these cases the resulting matroids are isomorphic to $M \oplus U_{0,1}$ and $M \oplus U_{1,1}$, respectively. Another type of well-defined extension is parallel extension of $M$. Here we add a new element in parallel to some existing element of $M$. For more details see [5, Section 7.2].

Now we prove Theorem 3.3.1.

Proof of Theorem 3.3.1. Assume that $M$ is decomposable. If $M$ is not connected, then Proposition 1.8.10(ii) tells us that either $M_{A}$ or $M_{B}$ is disconnected and hence decomposable, and the theorem holds. So let us assume that $M$ is connected. Let $N_{1}$ and $N_{2}$ be two matroids such that $M=N_{1} \vee N_{2}$. We also assume that $E\left(N_{1}\right)=E\left(N_{2}\right)=E(M)$.

If $M \mid A$ has a coloop, then by Lemma 3.3.4 above, $M_{A}$ is decomposable and we are done. So assume that neither $M \mid A$ nor $M \mid B$ have coloops. Since $M$ is connected, then $r(M)=r\left(N_{1}\right)+r\left(N_{2}\right)$ by Corollary 3.1.3. It also 
follows by Corollary 3.1.2 that

$$
r_{M}(A)=r_{N_{1}}(A)+r_{N_{2}}(A)
$$

Similarly,

$$
r_{M}(B)=r_{N_{1}}(B)+r_{N_{2}}(B) .
$$

Since $(A, B)$ is a 2 -separation of $M$, then

$$
\begin{aligned}
& r(M)=r_{M}(A)+r_{M}(B)-1 \\
& \quad=r_{N_{1}}(A)+r_{N_{2}}(A)+r_{N_{1}}(B)+r_{N_{2}}(B)-1 .
\end{aligned}
$$

The aforementioned tells us on one hand that either $r\left(N_{1}\right)$ equals $r_{N_{1}}(A)+$ $r_{N_{1}}(B)-1$, or it equals $r_{N_{1}}(A)+r_{N_{1}}(B)$. In the former $(A, B)$ is a 2separation of $N_{1}$, in the later $(A, B)$ is a 1-separation of $N_{1}$. On the other hand, either $r\left(N_{2}\right)$ equals $r_{N_{2}}(A)+r_{N_{2}}(B)-1$, or it equals $r_{N_{2}}(A)+r_{N_{2}}(B)$. In the former $(A, B)$ is a 2-separation of $N_{2}$, in the later $(A, B)$ is a 1separation of $N_{2}$.

Without loss of generality we assume from now on that $(A, B)$ is a 2 separation of $N_{1}$ and a 1-separation of $N_{2}$.

Define a single element extension $M^{\prime}$ of $M$ as follows. By Lemma 1.8.15 $M=P\left(M_{A}, M_{B}\right) \backslash p$. Let $M^{\prime}$ be $P\left(M_{A}, M_{B}\right)$. Then $M^{\prime}$ is a single element extension of $M$ with ground set $E\left(M^{\prime}\right)=E(M) \cup\{p\}$, as shown in Figure 3.1 .

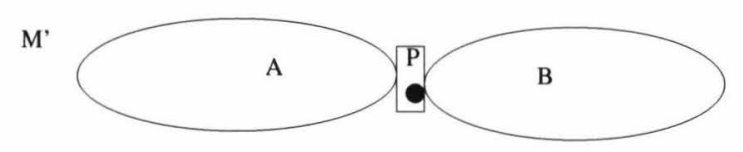

Figure 3.1: $M^{\prime}=P\left(M_{A}, M_{B}\right)$.

We now define two single element extensions $N_{1}^{\prime}$ and $N_{1}^{\prime}$ of $N_{1}$ and $N_{2}$ respectively. We have assumed that $(A, B)$ is a 2-separation of $N_{1}$, meaning $N_{1}$ is the 2 -sum of two matroids. Let $N_{1}$ be the 2 -sum of $N_{1 A}$ and $N_{1 B}$. So by Lemma 1.8.15 $N_{1}=P\left(N_{1 A}, N_{1 B}\right) \backslash p$. Let $N_{1}^{\prime}$ be $P\left(N_{1 A}, N_{1 B}\right)$, as shown in Figure 3.2. 


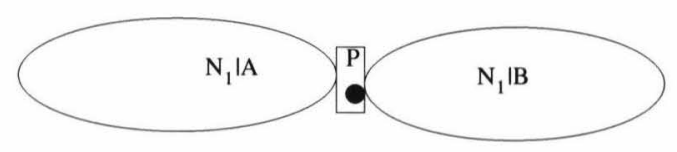

Figure 3.2: $N_{1}^{\prime}=P\left(N_{1 A}, N_{1 B}\right)$.

Since $(A, B)$ is a 1 -separation of $N_{2}$, then $N_{2}$ is the 1-sum of two matroids. So $N_{2}=\left(N_{2} \mid A\right) \oplus\left(N_{2} \mid B\right)$. Here the single element extension of $N_{2}$ giving $N_{2}^{\prime}$ is as follows. Add $\{p\}$ as a loop to $N_{2}$ and get the new matroid $N_{2}^{\prime}=\left(N_{2} \mid A\right) \oplus\left(N_{2} \mid B\right) \oplus U_{0,1}$, on the ground set $E\left(N_{2}^{\prime}\right)=E\left(N_{2}\right) \cup\{p\}$ as shown in Figure 3.3.

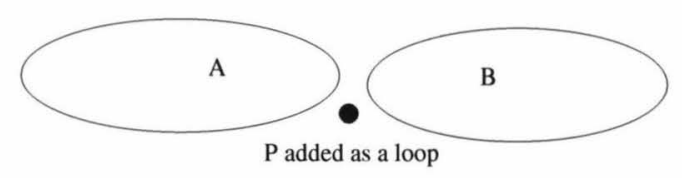

Figure 3.3: $N_{2}^{\prime}=\left(N_{2} \mid A\right) \oplus\left(N_{2} \mid B\right) \oplus U_{0,1}$.

Now that we have extended $M, N_{1}$, and $N_{2}$ to $M^{\prime}, N_{1}^{\prime}$ and $N_{2}^{\prime}$ respectively, we show that $M^{\prime}=N_{1}^{\prime} \vee N_{2}^{\prime}$. We will analyze the bases for both sides. We have two cases to consider, one where bases contain $p$ and the other where they do not.

Case 1. Consider the bases of $M^{\prime}=P\left(M_{A}, M_{B}\right)$ containing $p$. They are by Proposition 1.8.5(ii) of the form

$$
B=B_{A} \cup B_{B}
$$

where $B_{A}=B \cap E\left(M_{A}\right)$ is a basis of $M_{A}$ containing $p$, and $B_{B}=B \cap$ $E\left(M_{B}\right)$ is a basis of $M_{B}$ containing $p$. A basis of $N_{1}^{\prime} \vee N_{2}^{\prime}$ containing $p$ is of the form

$$
B^{\prime} \cup B^{\prime \prime},
$$

where $B^{\prime}$ is a basis of $N_{1}^{\prime}$, and $B^{\prime \prime}$ is a basis of $N_{2}^{\prime}$. Element $p$ cannot be in $B^{\prime \prime}$ as $p$ is a loop of $N_{2}^{\prime}$. Therefore $p \in B^{\prime}$.

A basis of $N_{1}^{\prime}=P\left(N_{1 A}, N_{1 B}\right)$ containing $p$ is of the form

$$
B^{\prime}=B_{A}^{\prime} \cup B_{B}^{\prime},
$$


where $B_{A}^{\prime}=B^{\prime} \cap E\left(N_{1 A}\right)$ is a basis of $N_{1 A}$ containing $p$, and $B_{B}^{\prime}=B^{\prime} \cap$ $E\left(N_{1 B}\right)$ is a basis of $N_{1 B}$ containing $p$. A basis of $N_{2}^{\prime}$ is of form

$$
B^{\prime \prime}=B_{A}^{\prime \prime} \cup B_{B}^{\prime \prime},
$$

where $B_{A}^{\prime \prime}=B^{\prime \prime} \cap E\left(N_{2} \mid A\right)$ is a basis of $N_{2} \mid A$, and $B_{B}^{\prime \prime}=B^{\prime \prime} \cap E\left(N_{2} \mid B\right)$ is a basis of $N_{2} \mid B$.

A basis of $N_{1}^{\prime} \vee N_{2}^{\prime}$ is by 3.3.6 and 3.3.7 $B^{\prime} \cup B^{\prime \prime}=B_{A}^{\prime} \cup B_{B}^{\prime} \cup B_{A}^{\prime \prime} \cup B_{B}^{\prime \prime}=$ $\left(B_{A}^{\prime} \cup B_{A}^{\prime \prime}\right) \cup\left(B_{B}^{\prime} \cup B_{B}^{\prime \prime}\right)$. Since $\left(N_{1 A}\right) \cup\left(N_{2} \mid A\right)=M_{A}$, and $\left(N_{1 B}\right) \cup$ $\left(N_{2} \mid B\right)=M_{B}$, then $\left(B_{A}^{\prime} \cup B_{A}^{\prime \prime}\right)$ is a basis of $M_{A}$, and $\left(B_{B}^{\prime} \cup B_{B}^{\prime \prime}\right)$ is a basis of $M_{B}$. Therefore $B=B_{A} \cup B_{B}=B^{\prime} \cup B^{\prime \prime}$, and so the bases of $M^{\prime}$ and $N_{1}^{\prime} \vee N_{2}^{\prime}$ are equal.

Case 2. Now consider the bases that do not contain $p$. Those bases of $M^{\prime}=P\left(M_{A}, M_{B}\right)$ are by Proposition 1.8.5(ii) of the form

$$
B=\left(B_{A} \cup B_{B}\right)-\{p\},
$$

where $B_{A}=\left(B \cap E\left(M_{A}\right)\right) \cup\{p\}$ is a basis of $M_{A}$ containing $p$, and $B_{B}=$ $B \cap E\left(M_{B}\right)$ is a basis of $M_{B}$ not containing $p$. Note that we lose no generality if $p$ was in $B_{B}$ and not in $B_{A}$.

Bases of $N_{1}^{\prime}=P\left(N_{1 A}, N_{1 B}\right)$ are

$$
B^{\prime}=\left(B_{A}^{\prime} \cup B_{B}^{\prime}\right)-\{p\},
$$

where $B_{A}^{\prime}=\left(B^{\prime} \cap E\left(N_{1 A}\right)\right) \cup\{p\}$ is a basis of $N_{1 A}$ containing $p$, and $B_{B}^{\prime}=$ $\left(B^{\prime} \cap E\left(N_{1 B}\right)\right)$ is a basis of $N_{1 B}$ not containing $p$. Again we lose no generality if $p$ was in $B_{B}^{\prime}$ not $B_{A}^{\prime}$.

As for the bases of $N_{2}^{\prime}$, they are of the form

$$
B^{\prime \prime}=B_{A}^{\prime \prime} \cup B_{B}^{\prime \prime}
$$

not containing $p$, where $B_{A}^{\prime \prime}=\left(B^{\prime \prime} \cap A\right)$ is a basis of $\left(N_{2} \mid A\right)$, and $B_{B}^{\prime \prime}=$ $\left(B^{\prime \prime} \cap B\right)$ is a basis of $\left(N_{2} \mid B\right)$. We see that a basis of $N_{1}^{\prime} \vee N_{2}^{\prime}$ not containing $p$ by 3.3.9 and 3.3.10 is $B^{\prime} \cup B^{\prime \prime}=\left(B_{A}^{\prime} \cup B_{B}^{\prime} \cup B_{A}^{\prime \prime} \cup B_{B}^{\prime \prime}\right)-\{p\}=\left(B_{A}^{\prime} \cup\right.$ $\left.B_{A}^{\prime \prime}\right) \cup\left(B_{B}^{\prime} \cup B_{B}^{\prime \prime}\right)-\{p\}$. Which is equal to $\left(B_{A} \cup B_{B}\right)-\{p\}$ as discussed in Case 1. From Cases 1 and 2 we conclude that $M^{\prime}=N_{1}^{\prime} \vee N_{2}^{\prime}$. 
If every element of $E(M)$ is either a loop in $N_{1}$ or a loop in $N_{2}$, then $N_{1} \vee N_{2}$ will be equivalent to a direct sum and hence disconnected, giving a contradiction. So there must exist an element $z \in E(M)$, such that $z$ is not a loop in $N_{1}$ and not a loop in $N_{2}$. So without loss of generality we assume that $z \in A$. Since $M^{\prime}=N_{1}^{\prime} \vee N_{2}^{\prime}$, then $M^{\prime} \backslash B=\left(N_{1}^{\prime} \backslash B\right) \vee\left(N_{2}^{\prime} \backslash B\right)$ by Proposition 2.4.1(i), where

$$
M^{\prime} \backslash B=M_{A} .
$$

Element $z \in A$ and $z$ is not a loop. Also $A \subseteq E\left(N_{1}^{\prime} \backslash B\right)$, and $A \subseteq E\left(N_{2}^{\prime} \backslash\right.$ $B$ ) as shown in Figures 3.2 and 3.3. So it follows that neither $N_{1}^{\prime} \backslash B$ nor $N_{2}^{\prime} \backslash B$ consists entirely of loops. Therefore it follows from 3.3 .11 and Proposition 2.4.1(ii) that $M_{A}$ is decomposable. Therefore we conclude that if $M=M_{A} \oplus_{2} M_{B}$ is decomposable, then at least one of $M_{A}$ and $M_{B}$ is decomposable.

The converse of Theorem 3.3.1 is not necessarily correct as we shall see shortly. The next theorem, however, is a weaker result.

Theorem 3.3.5. Let $M=M_{A} \oplus_{2} M_{B}$, such that $\{p\}=E\left(M_{A}\right) \cap E\left(M_{B}\right)$. If one of $M_{A}$ and $M_{B}$ is decomposable, say $M_{A}$, such that $M_{A}=N_{1} \vee N_{2}$, and $p \notin\left[E\left(N_{1}\right) \cap E\left(N_{2}\right)\right]$, then $M$ is decomposable.

Proof. If $M$ is disconnected, then it is decomposable. So assume that $M$ is connected. By Proposition 1.8.10, both $M_{A}$ and $M_{B}$ are connected, therefore neither of them has a coloop. Suppose without loss of generality that $p \in$ $E\left(N_{2}\right)$, and $p \notin E\left(N_{1}\right)$. By Lemma 1.8.15, $M=S\left(M_{A}, M_{B}\right) / p$. Since we have $M=\left(N_{1} \vee N_{2}\right) \oplus_{2} M_{B}$, then Proposition 3.2.1 gives

$$
M=S\left(\left(N_{1} \vee N_{2}\right), M_{B}\right) / p=\left[\left(N_{1} \vee N_{2}\right) \vee M_{B}\right] / p .
$$

That in turn by Proposition 2.3.2 is equal to

$$
\left[N_{1} \vee\left(N_{2} \vee M_{B}\right)\right] / p
$$


Since $p$ is not in $E\left(N_{1}\right)$, then by Proposition $2.4 .5,3.3 .13$ is equal to

$$
N_{1} \vee\left[\left(N_{2} \vee M_{B}\right) / p\right]
$$

We already know that $N_{1}$ and $N_{2}$ do not consist entirely of loops. Since $N_{2}$ and $M_{B}$ have element $p$ in common, then $p$ cannot be in parallel with any element in $N_{2} \vee M_{B}$, meaning contracting $p$ from $N_{2} \vee M_{B}$ can never make $\left(N_{2} \vee M_{B}\right) / p$ consist entirely of loops. So $\left(N_{2} \vee M_{B}\right) / p$ does not consist entirely of loops. Then it follows from Proposition 2.4.1(ii) that $M$ is decomposable.

To see why the converse of Theorem 3.3.1 may not work, consider the following example.

Example 3.3.6. Let $M_{1}, M_{2}$ and $M_{3}$ be three matroids, such that $M_{1} \cong$ $M_{2} \cong M_{3} \cong F_{7}$, where element $p$ is the only element they have in common. Consider $S\left(M_{1}, M_{2}\right) \oplus_{2} M_{3}$ as shown in Figure 3.4. $S\left(M_{1}, M_{2}\right)$ is a matroid union by Proposition 3.2.1. Moreover, since the series connection of two binary matroids is also binary by Proposition 1.8.14, then $S\left(M_{1}, M_{2}\right)$ is binary. Also, since $F$-representable matroids are closed under the operation of 2-sum by Proposition 1.8.11, then $S\left(M_{1}, M_{2}\right) \oplus_{2} M_{3}$ is binary too. Every element now in this 2-connected binary matroid $S\left(M_{1}, M_{2}\right) \oplus_{2} M_{3}$ is fixed, a notion that will be explained in the next chapter. In Section 4.4.1, Theorem 4.4.3 shows why this structure gives an indecomposable matroid.

\subsection{When is the union 3-connected?}

In this section we characterize when the union of two matroids is 3-connected under the assumption that there are two elements in common in their ground sets. First we need a definition. Let $x$ and $y$ be elements of the connected matroid $M$. Then $x$ and $y$ are split if there is no 2-separation $(A, B)$ in $M$ such that $x, y \in A$ or $x, y \in B$. In other words, whenever you see a 2 -separation in $M$, then $x$ is never in the same part of the 2-separation as $y$. Note that, if $M$ is 3 -connected, then every two elements are split. The following theorem characterizes when the union is 3-connected, in the case 


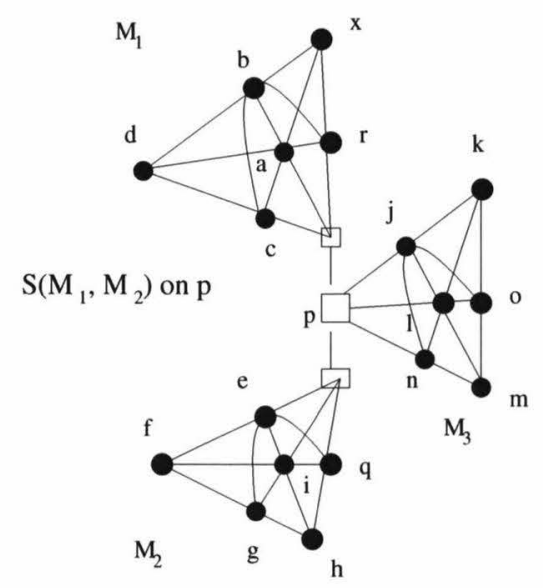

Figure 3.4: $S\left(M_{1}, M_{2}\right) \oplus_{2} M_{3}$.

that $E\left(M_{1}\right)$ and $E\left(M_{2}\right)$ intersect in two elements, it is the main theorem of this section.

Theorem 3.4.1. Let $M_{1}$ and $M_{2}$ be two loopless matroids with $E\left(M_{1}\right) \cap$ $E\left(M_{2}\right)=\{x, y\} . M_{1} \vee M_{2}$ is 3-connected if and only if the following hold:

(i) $M_{1}$ and $M_{2}$ are connected with no series pairs.

(ii) $\left|E\left(M_{1}\right)\right|,\left|E\left(M_{2}\right)\right| \geq 3$ and

(iii) $x$ and $y$ are split in $M_{1}$ and split in $M_{2}$.

The example below illustrates Theorem 3.4.1, that the union of two matroids is 3-connected if and only if the two matroids satisfy conditions (i)-(iii) in Theorem 3.4.1.

Example 3.4.2. Let $M_{1}$ be the matroid on $E\left(M_{1}\right)=\{a, b, c, x, y, h, g\}$, and $M_{2}$ be a matroid on $E\left(M_{2}\right)=\{f, e, d, x, y, i, j\}$ as shown in Figure 3.5(a). It is seen that neither $M_{1}$ nor $M_{2}$ is disconnected, and neither of them has a series pair. Moreover, $x$ and $y$ are split in both $M_{1}$ and $M_{2}$. As shown in Figure 3.5(b), $M_{1} \vee M_{2}$ is the rank-6 whirl $\mathcal{W}^{6}$, which is 3-connected.

To prove Theorem 3.4.1, we first establish some lemmas. The first is elementary.

Lemma 3.4.3. Let $x$ be an element of the matroid $M$. If $C^{*}$ contains a cocircuit of $M \backslash x$, then $C^{*} \cup x$ contains a cocircuit of $M$. 


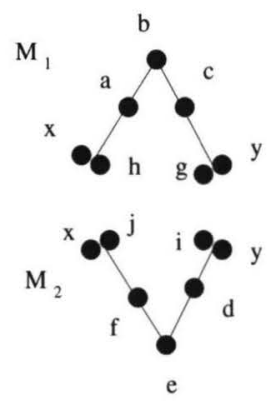

(a)

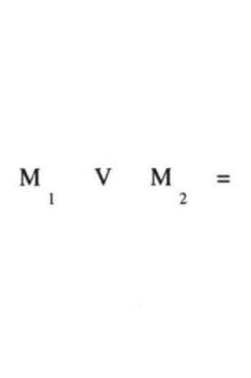

The rank-6 whirl is 3 -connected

(b)

Figure 3.5: A 3-connected matroid union.

In the next lemma we make no assumptions about the cardinality of the intersections of the ground sets of $M_{1}$ and $M_{2}$.

Lemma 3.4.4. Let $M_{1}$ and $M_{2}$ be matroids. If $C^{*}$ is a cocircuit of $M_{1}$ or $M_{2}$, then $C^{*}$ contains a cocircuit of $M_{1} \vee M_{2}$.

Proof. Assume $C^{*}$ is a cocircuit of $M_{1}$. If $\left|C^{*}\right|=1$, then the lemma follows from Lemma 3.2.2. Thus we may assume that $\left|C^{*}\right|=k$ and, for induction, that the lemma holds for any cocircuit of $M_{1}$ with $k-1$ elements. Say $z \in C^{*}$, then $C^{*}-\{z\}$ is a cocircuit of $M_{1} \backslash z$. If $z \in E\left(M_{2}\right)$, then $M \backslash$ $z=\left(M_{1} \backslash z\right) \vee\left(M_{2} \backslash z\right)$ by Proposition 2.4.1, and if $z \notin E\left(M_{2}\right)$, then $M \backslash z=\left(M_{1} \backslash z\right) \vee\left(M_{2}\right)$ by Proposition 2.4.2. In either case it follows from the induction assumption that $C^{*}-\{z\}$ contains a cocircuit of $M \backslash z$. The lemma now follows from Lemma 3.4.3.

Now we consider the different obstacles preventing $M=M_{1} \vee M_{2}$ from being 3-connected.

Lemma 3.4.5. Let $M=M_{1} \vee M_{2}$, where $M_{1}$ and $M_{2}$ are two matroids with $\{x, y\}=E\left(M_{1}\right) \cap E\left(M_{2}\right)$. If $M_{1}$ or $M_{2}$ is disconnected, then $M$ is not 3-connected.

Proof. Without loss of generality, suppose that $M_{1}$ is disconnected. Suppose that $M_{1}$ has two components $N_{1}$ and $N_{2}$, so that $M_{1}=N_{1} \oplus N_{2}=N_{1} \vee N_{2}$. There are two cases to consider. 
Case 1. The elements $x, y$ are in one component of $M_{1}$, say $N_{1}$ without loss of generality. Note that $N_{1}$ and $M_{2}$ have $x$ and $y$ in common as shown in Figure 3.6, so let $N_{1} M_{2}=N_{1} \vee M_{2}$. It follows by Propositions 2.3.2 and 2.3.1 that

$$
M=M_{1} \vee M_{2}=\left(N_{2} \vee N_{1}\right) \vee M_{2}=N_{2} \vee\left(N_{1} \vee M_{2}\right)
$$

Sine $N_{2}$ and $N_{1} M_{2}$ are disjoint, then 3.4 .1 is equal to $N_{2} \oplus N_{1} M_{2}$. Therefore $M$ is disconnected and hence not 3-connected.

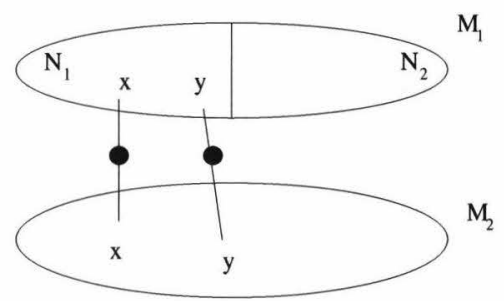

Figure 3.6: Intersection elements in one component.

Case 2. The element $x$ is in one component of $M_{1}$, say $N_{1}$, and $y$ is in the other component $N_{2}$ as shown in Figure 3.7. Let $M_{12}=N_{1} \vee M_{2}$. Since $E\left(N_{1}\right) \cap E\left(M_{2}\right)=\{x\}$, then by Proposition 3.2.1 $M_{12}=S\left(N_{1}, M_{2}\right)$. By Propositions 2.3.2 and 2.3.1, we get

$$
\begin{aligned}
M=M_{2} \vee\left(N_{1} \vee N_{2}\right)=\left(M_{2} \vee N_{1}\right) & \vee N_{2} \\
= & S\left(M_{2}, N_{1}\right) \vee N_{2}=M_{12} \vee N_{2} .
\end{aligned}
$$

Since $E\left(M_{12}\right) \cap E\left(N_{2}\right)=\{y\}$, then $M_{12} \vee N_{2}=S\left(M_{12}, N_{2}\right)$. But $M_{12} \vee N_{2}=$ $M$, so $M$ is a series connection, showing $M$ is not 3-connected.

Lemma 3.4.6. If $M=M_{1} \vee M_{2}$ is such that at least one of $M_{1}$ and $M_{2}$ is isomorphic to $U_{2,3}$, and $\{x, y\}=E\left(M_{1}\right) \cap E\left(M_{2}\right)$, then $M$ is not 3connected.

Proof. Suppose without loss of generality that $M_{1} \cong U_{2,3}$. Since $x \in$ $E\left(M_{1}\right) \cap E\left(M_{2}\right)$, then by Proposition 2.4.1 $M \backslash x=\left(M_{1} \backslash x\right) \vee\left(M_{2} \backslash x\right)$. Now $M_{1} \backslash x$ and $M_{2} \backslash x$ have only the element $y$ in common. Therefore by 


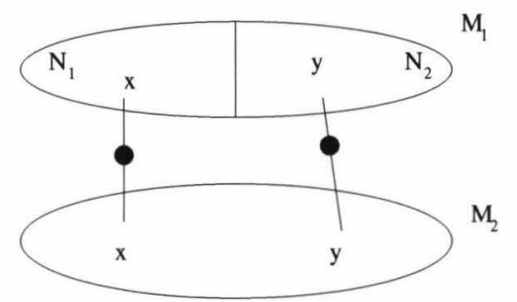

Figure 3.7: One intersection element in each component.

Proposition 3.2.1 $\left(M_{1} \backslash x\right) \vee\left(M_{2} \backslash x\right)=S\left(M_{1} \backslash x, M_{2} \backslash x\right)$. But $M_{1} \backslash x$ is disconnected, and hence by Proposition 1.8.8, $M \backslash x=S\left(M_{1} \backslash x, M_{2} \backslash x\right)$ is also disconnected. It follows from Proposition 1.8.16 that $M$ cannot be 3-connected.

The reason $M$ was not 3-connected in Lemma 3.4.6 above, even though $M_{1}$ was 3 -connected, is that $U_{2,3}$ has series pairs. So using Lemma 3.4.4 presented earlier, we can generalize Lemma 3.4.6 to the following:

Lemma 3.4.7. If $M=M_{1} \vee M_{2}$ is such that $|E(M)| \geq 4, M_{1}$ or $M_{2}$ has a series pair, and $E\left(M_{1}\right) \cap E\left(M_{2}\right)=\{x, y\}$, then $M$ is not 3-connected.

Proof. Suppose without loss of generality that $M_{1}$ has a series pair $C_{1}^{*}=$ $\{s, t\}$. Since a series pair is a 2-element cocircuit, then by Lemma 3.4.4, $C_{1}^{*}$ contains a cocircuit $C^{*}$ in the matroid union $M$. But $C_{1}^{*}$ only has two elements. So either $C^{*}$ itself is a series pair in $M$, implying that $M$ can be disconnected by removing one of the elements $s$ and $t$ from $C^{*}$. Hence by Proposition 1.8.16 $M$ is not 3 -connected, or $C^{*}$ contains a 1-element cocircuit, that is a coloop. Showing that $M$ is not 3-connected.

The results above are used in proving Theorem 3.4.1 part (i). The next Lemma is for proving part (ii).

Lemma 3.4.8. Let $M=M_{1} \vee M_{2}$ is such that $|E(M)| \geq 4, E\left(M_{1}\right) \cap$ $E\left(M_{2}\right)=\{x, y\}$, where $M_{1}$ and $M_{2}$ are two loopless matroids. If one of $E\left(M_{1}\right)$ and $E\left(M_{2}\right)$ has cardinality 2 or less, then $M$ is not 3-connected.

Proof. Suppose without loss of generality that $\left|E\left(M_{1}\right)\right| \leq 2$. $\left|E\left(M_{1}\right)\right|$ cannot be one or zero, because $M_{1}$ and $M_{2}$ must have exactly two elements in 
common. So assume $\left|E\left(M_{1}\right)\right|=2$, so that $E\left(M_{1}\right)=\{x, y\}$. Consider $\left(M_{1} \vee M_{2}\right) \backslash x$. By Proposition 2.4.1 $\left(M_{1} \vee M_{2}\right) \backslash x=\left(M_{1} \backslash x\right) \vee\left(M_{2} \backslash x\right)$. But now $M_{1} \backslash x$ has only one element left in it which is $y$. Since $M_{1}$ is loopless, then $y$ is not a loop in $M_{1}$, so $y$ is independent in $M_{1}$, and hence it is in every basis of $M \backslash x$. Meaning $y$ is a coloop of $M \backslash x$. Therefore by Proposition 1.5.2 $M \backslash x$ is disconnected. This shows by Proposition 1.8.16 that $M$ cannot be 3-connected.

The next two Lemmas will be used to prove Theorem 3.4.1 Part (iii).

Lemma 3.4.9. Let $M=M_{1} \vee M_{2}$ be a connected matroid, with $E\left(M_{1}\right) \cap$ $E\left(M_{2}\right)=\{x, y\}$, where both $M_{1}$ and $M_{2}$ are connected, neither of them has a series pair, and $\left|E\left(M_{1}\right)\right|,\left|E\left(M_{2}\right)\right| \geq 3$. If at least one of $M_{1}$ and $M_{2}$ does not split $x$ and $y$, then $M$ is not 3-connected.

Proof. Suppose without loss of generality that $M_{1}$ does not split $x$ and $y$, so that it has a partition $(A, B)$ such that $x, y \in A$ as shown in Figure 3.8. Then

$$
r\left(M_{1}\right)=r_{M_{1}}(A)+r_{M_{1}}(B)-1
$$

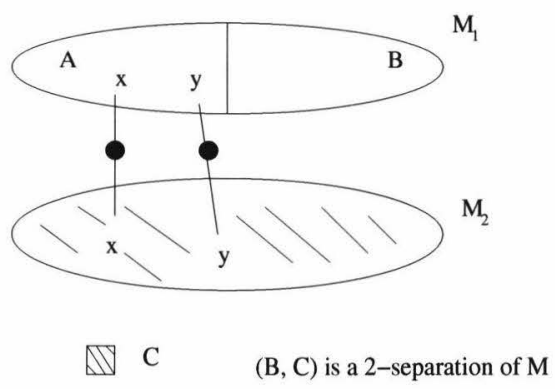

Figure 3.8: When is the union not 3-connected?

Say $E\left(M_{2}\right)=C$. So $C \cap A=\{x, y\}$. Now, as $M$ is connected, then by Corollary 3.1.3 $r(M)=r\left(M_{1}\right)+r\left(M_{2}\right)$. But since $M_{2} \mid C=M_{2}$, then by Corollary 2.4.3 $M \mid(A \cup C)=\left(M_{1} \mid A\right) \vee\left(M_{2} \mid C\right)=M_{2} \vee\left(M_{1} \mid A\right)$. By Lemma 1.2 .1

$$
r_{M}(A \cup C) \leq r\left(M_{2}\right)+r_{M_{1}}(A) .
$$


Also by Lemma 2.4.4

$$
M\left|B=M_{1}\right| B
$$

Hence by 3.4 .3 we get

$$
r(M)=r\left(M_{1}\right)+r\left(M_{2}\right)=r_{M_{1}}(A)+r_{M_{1}}(B)-1+r\left(M_{2}\right),
$$

and that by 3.4 .4 gives

$$
\begin{aligned}
r_{M_{1}}(A)+r_{M_{1}}(B)-1+r\left(M_{2}\right) \geq & r_{M}(A \cup C)+r_{M_{1}}(B)-1 \\
& =r_{M}(A \cup C)+r_{M}(B)-1 .
\end{aligned}
$$

But by Lemma 1.2.1 $r(M) \leq r_{M}(A \cup C)+r_{M}(B)$. Therefore $(A \cup C, B)$ is either a 2-separation or 1-separation of $M$. As $M$ is connected, then it must be that $(A \cup C, B)$ is a 2 -separation of $M$. Hence $M$ is not 3connected.

For the converse of Theorem 3.4.1, we have the following:

Lemma 3.4.10. Let $M=M_{1} \vee M_{2}$, where both $M_{1}$ and $M_{2}$ are connected, $\{x, y\}=E\left(M_{1}\right) \cap E\left(M_{2}\right)$, neither $M_{1}$ nor $M_{2}$ has a series pair, and $\left|E\left(M_{1}\right)\right|,\left|E\left(M_{2}\right)\right| \geq 3$. If neither $M_{1}$ nor $M_{2}$ splits $x$ and $y$, then $M$ is 3-connected.

The proof will use the following lemmas.

Lemma 3.4.11. Let $N$ be a connected matroid with no series pairs. If $\{x, y\} \subseteq E(N)$, such that $x$ and $y$ are split in $N$, then $N \backslash x$ is connected.

Proof. Assume that $N \backslash x$ is disconnected. Let $(A, B)$ be a separation of $N \backslash x$, where say $y \in A$. Then $(A \cup\{x\}, B)$ is a 2-separation of $N$ unless $|B|=1$. But if $|B|=1$, then $B \cup\{x\}$ is a series pair in $N$, contradicting the fact that $N$ has no series pairs. But now both $x$ and $y$ are in $A \cup\{x\}$, meaning $x$ and $y$ are not split in $N$, contradiction. Therefore $N \backslash x$ is connected.

Lemma 3.4.12. Let $N$ be a connected matroid with no series pairs. Let $(A, B)$ be a 2-separation of $N$, such that $z \in A$. Then $z$ is a coloop of $N \mid A$ 
if and only if $N \backslash z$ is not connected, where $(A-\{z\}, B)$ is a separation of $N \backslash z$. Moreover, if the above holds, then $(A-\{z\}, B \cup\{z\})$ is a 2-separation of $N$.

Proof. Since $(A, B)$ is a 2-separation of $N$, then $r(A)+r(B)-1=r(N)$. If $z$ is a coloop of $N \mid A$, then $r(A-\{z\})=r(A)-1$. So that $r(A-\{z\})=$ $r(N)-r(B)+1-1=r(N)-r(B)$. Therefore $N \backslash z$ is disconnected, where $(A-\{z\}, B)$ is a separation of $N \backslash z$. Conversely, if $N \backslash z$ is disconnected, then $(A-\{z\}, B)$ is a separation of $N \backslash z$. So that $r(A-\{z\})+r(B)=r(N)$. But $r(A)+r(B)-1=r(N)$. Meaning $r(A-\{z\})=r(A)-1$. Implying that $z$ is a coloop of $N \mid A$. Moreover, if $|A|=2$, then $(A-\{z\}, B \cup\{z\})$ is not a 2-separation of $N$. But if $|A|=2$ and $(A-\{z\}, B)$ is a separation of $N \backslash z$, then $z$ is in a series pair in $N$, contradicting the fact that $N$ has no series pairs.

Proof of Lemma 3.4.10. By Lemma 3.4.11, both $M_{1} \backslash x$ and $M_{2} \backslash x$ are connected. By Proposition 3.2.1 $\left(M_{1} \backslash x\right) \vee\left(M_{2} \backslash x\right)=S\left(M_{1} \backslash x, M_{2} \backslash x\right)$. So that by Proposition 1.8.8, $\left(M_{1} \backslash x\right) \vee\left(M_{2} \backslash x\right)$ is also connected. By Proposition 2.4.1 $M \backslash x=\left(M_{1} \backslash x\right) \vee\left(M_{2} \backslash x\right)$, so that $M \backslash x$ is connected. Similarly, $M \backslash y$ is connected. Since $M_{1}$ and $M_{2}$ are both connected, then neither $M_{1}$ nor $M_{2}$ have loops or coloops, therefore $M$ does not have loops or coloops. Hence $M$ is connected. But because $M \backslash x$ is also connected, then $x$ is not in a series pair with any other element.

Now suppose that $M$ has a 2 -separation $(A, B)$. Let $A_{1}=A \cap$ $E\left(M_{1}\right), A_{2}=A \cap E\left(M_{2}\right), B_{1}=B \cap E\left(M_{1}\right)$ and $B_{2}=B \cap E\left(M_{2}\right)$. We have two cases to consider.

Case 1. $\{x, y\} \subseteq A$ or $\{x, y\} \subseteq B$. Without loss of generality, assume that $\{x, y\} \subseteq A$. By Lemmas 3.4.11 and 3.4.12, neither $x$ nor $y$ is a coloop of $M \mid A$. Hence we may assume that $M \mid A$ has no coloops. So that by Corollary 3.1.2 we have

$$
r(M \mid A)=r\left(M_{1} \mid A_{1}\right)+r\left(M_{2} \mid A_{2}\right) .
$$

But as $\{x, y\} \cap B=\emptyset$, then

$$
r(M \mid B)=r\left(M_{1} \mid B_{1}\right)+r\left(M_{2} \mid B_{2}\right) .
$$


By 3.4.8 and 3.4.9 we have

$$
\begin{aligned}
r(M) & =r(M \mid A)+r(M \mid B)-1 \\
& =r\left(M_{1} \mid A_{1}\right)+r\left(M_{2} \mid A_{2}\right)+r\left(M_{1} \mid B_{1}\right)+r\left(M_{2} \mid B_{2}\right)-1 .
\end{aligned}
$$

But since $M$ has no coloops, then by Corollary 3.1.2 $r(M)=r\left(M_{1}\right)+r\left(M_{2}\right)$. So that

$$
\begin{aligned}
r\left(M_{1}\right)+r\left(M_{2}\right)=r\left(M_{1} \mid A_{1}\right)+ & r\left(M_{2} \mid A_{2}\right) \\
& +r\left(M_{1} \mid B_{1}\right)+r\left(M_{2} \mid B_{2}\right)-1 .
\end{aligned}
$$

It may be that some of the subsets $A_{1}, A_{2}, B_{1}$ and $B_{2}$ are empty. Without loss of generality we consider the two following cases.

Case 1.1. $B_{1}=\emptyset$. If $B_{1}=\emptyset$, and $A_{1}$ is also empty, then $E\left(M_{1}\right)=$ $A_{1} \cup B_{1}$ would be empty, giving a contradiction. Therefore $A_{1}$ is not empty. Also, if $B_{2}$ is empty, then $B=B_{1} \cup B_{2}$ is empty. But $(A, B)$ is a 2separation of $M$, meaning $|B|$ must be at least 2, giving a contradiction. Therefore $B_{2}$ is not empty. Finally if $A_{2}$ is empty, then $r\left(M_{1}\right)+r\left(M_{2}\right)=$ $r\left(M_{1} \mid A_{1}\right)+r\left(M_{2} \mid B_{2}\right)-1$. But since $B_{1}$ is empty, then $M_{1} \mid A_{1}$ must be equal to $M_{1}$. Similarly, $M_{2} \mid B_{2}$ must be equal to $M_{2}$, giving a contradiction. Therefore if $B_{1}$ is empty, then it must be that $r\left(M_{1}\right)+r\left(M_{2}\right)=r\left(M_{1} \mid A_{1}\right)+$ $r\left(M_{2} \mid A_{2}\right)+r\left(M_{2} \mid B_{2}\right)-1$. Showing that $\left(A_{2}, B_{2}\right)$ is a 2-separation of $M_{2}$ that does not split $x$ and $y$, contradicting the assumption that $x$ and $y$ are split in $M_{2}$.

Case 1.2. $B_{1} \neq \emptyset$. If any of the other subsets are empty, then we get the same contradiction shown in Case 1.1. So suppose that none of the subsets are empty. Then either $r\left(M_{1}\right)=r\left(M_{1} \mid A_{1}\right)+r\left(M_{1} \mid B_{1}\right)$, showing that $\left(A_{1}, B_{1}\right)$ is a separation of $M_{1}$. Or $r\left(M_{2}\right)=r\left(M_{2} \mid A_{2}\right)+r\left(M_{2} \mid B_{2}\right)$, showing that $\left(A_{2}, B_{2}\right)$ is a separation of $M_{2}$. Both possibilities contradict the fact that both $M_{1}$ and $M_{2}$ are connected.

Case 2. Assume without loss of generality that $x \in A, y \in B$. Suppose that $z$ is a coloop of $M \mid A$. Since neither $x$ nor $y$ are coloops of $M \mid A$, then $z \neq x$. As $M$ has no series pairs, then $M \backslash z$ has no coloops. Assume that 
$z \in E\left(M_{1}\right)$. Then by Corollary 3.1 .2 we have

$$
r_{M \backslash z}(A-\{z\})=r_{M_{1}}\left(A_{1}-\{z\}\right)+r_{M_{2}}\left(A_{2}\right),
$$

and

$$
r_{M \backslash z}(B)=r_{M_{1}}\left(B_{1}\right)+r_{M_{2}}\left(B_{2}\right) .
$$

But

$$
\begin{aligned}
r(M \backslash z)= & r_{M \backslash z}(A-\{z\})+r_{M \backslash z}(B) \\
= & r_{M_{1}}\left(A_{1}-\{z\}\right)+r_{M_{2}}\left(A_{2}\right)+ \\
& r_{M_{1}}\left(B_{1}\right)+r_{M_{2}}\left(B_{2}\right) \\
& =r\left(M_{1} \backslash z\right)+r\left(M_{2}\right) .
\end{aligned}
$$

This shows that $\left(A_{2}, B_{2}\right)$ is a separation of $M_{2}$, contradicting the fact that $M_{2}$ is connected. Thus $M \mid A$, and similarly $M \mid B$ have no coloops. Therefore

$$
\begin{aligned}
& r_{M}(A)=r_{M_{1}}\left(A_{1}\right)+r_{M_{2}}\left(A_{2}\right) . \\
& r_{M}(B)=r_{M_{1}}\left(B_{1}\right)+r_{M_{2}}\left(B_{2}\right) .
\end{aligned}
$$

So that

$$
\begin{aligned}
r(M)=r_{M}(A) & +r_{M}(B)-1=r\left(M_{1}\right)+r\left(M_{2}\right) \\
& =r_{M_{1}}\left(A_{1}\right)+r_{M_{2}}\left(A_{2}\right)+r_{M_{1}}\left(B_{1}\right)+r_{M_{2}}\left(B_{2}\right)-1 .
\end{aligned}
$$

But since $x \in A_{1} \cap A_{2}$ and $x \in B_{1} \cap B_{2}$, then none of the subsets $A_{1}, A_{2}$, $B_{1}$ or $B_{2}$ is empty. It follows that one of $M_{1}$ and $M_{2}$ has a separation, contradicting the fact that they are both connected. So we conclude that $M$ has no 2-separation.

Now by combining all the aforementioned results we have a proof for Theorem 3.4.1.

Proof of Theorem 3.4.1. Let $M_{1}$ and $M_{2}$ be two loopless matroids with $E\left(M_{1}\right) \cap E\left(M_{2}\right)=\{x, y\}$. If $M_{1} \vee M_{2}$ is 3-connected, then: (i) If at least one of $M_{1}$ and $M_{2}$ is disconnected, then by Lemma 3.4.5 $M$ is not 3-connected, giving a contradiction. Therefore both $M_{1}$ and $M_{2}$ must be connected. If 
at least one of $M_{1}$ and $M_{2}$ has a series pair, then by Lemma 3.4.7 $M$ is not 3-connected, also giving a contradiction. Therefore neither $M_{1}$ nor $M_{2}$ can have a series pair. (ii) If at least one of $E\left(M_{1}\right)$ and $E\left(M_{2}\right)$ has size less than 3, then by Lemma 3.4.8 $M$ is not 3-connected, giving a contradiction. So it must be that $\left|E\left(M_{1}\right)\right|,\left|E\left(M_{2}\right)\right| \geq 3$. (iii) If at least one of $M_{1}$ and $M_{2}$ does not split $x$ and $y$, then Lemma 3.4.9 implies that $M$ is not 3-connected, giving a contradiction. So $x$ and $y$ must be split in $M_{1}$ and split in $M_{2}$.

Conversely, suppose both $M_{1}$ and $M_{2}$ are loopless connected matroids, neither of them has a series pair, $x$ and $y$ are split in $M_{1}$ and $M_{2}$, and $\left|E\left(M_{1}\right)\right|,\left|E\left(M_{2}\right)\right| \geq 3$. If $M_{1} \vee M_{2}$ was not 3-connected, then by Lemma 3.4.10 at least one of $M_{1}$ and $M_{2}$ does not split $x$ and $y$, giving a contradiction. Hence $M$ is 3-connected. 


\section{Chapter 4}

\section{Freedom of elements}

\subsection{Fixed elements and clones}

Certain elements of a matroid have 'freedom', while others are 'fixed'. These properties of elements will be used a lot in the material to come, in this chapter and the next. So in this section we present the basic facts about fixed elements and clones. For more details see [3, Sections 2 and 4].

Elements $x$ and $x^{\prime}$ of a matroid $M$ are clones if the map that interchanges $x$ and $x^{\prime}$ and acts as the identity on $E(M)-\left\{x, x^{\prime}\right\}$ is an automorphism of $M$. In other words, $x$ and $x^{\prime}$ are clones if they are indistinguishable up to labeling. An element $z$ of $M$ is fixed in $M$ if there is no single-element extension of $M$ by $z^{\prime}$ in which $z$ and $z^{\prime}$ are independent clones. Dually, the element $z$ is cofixed in $M$ if there is no single-element coextension of $M$ by $z^{\prime}$ in which $z$ and $z^{\prime}$ are coindependent clones.

Let $x$ be an element of a matroid $M$. The matroid $M^{\prime}$ is obtained by cloning $x$ with $x^{\prime}$ if $M^{\prime}$ is a single-element extension of $M$ by $x^{\prime}$, and $x$ and $x^{\prime}$ are clones in $M^{\prime}$.

If $x$ is not fixed, then there is a matroid $M^{\prime}$ obtained by cloning $x$ with $x^{\prime}$ such that $\left\{x, x^{\prime}\right\}$ is independent in $M^{\prime}$. We say that $M^{\prime}$ is obtained by independently cloning $x$ with $x^{\prime}$.

Proposition 4.1.1. [3, Section 4] If $\left\{x, x^{\prime}\right\}$ is a pair of loops, a pair of coloops, a parallel pair, or a series pair, then $x$ and $x^{\prime}$ are clones.

Next we state equivalent conditions for $x$ and $x^{\prime}$ to be clones in a matroid 
$M$, and then state further properties of fixed and non-fixed elements.

Proposition 4.1.2. [3, Proposition 4.2]. Let $x$ and $x^{\prime}$ be elements of a matroid $M$. Then the following are equivalent.

(i) $x$ and $x^{\prime}$ are clones in $M$.

(ii) The function that replaces $x$ by $x^{\prime}$ and acts as the identity on other elements is an isomorphism from $M \backslash x$ to $M \backslash x^{\prime}$.

(iii) $M / x \backslash x^{\prime}=M / x^{\prime} \backslash x$ and $r(\{x\})=r\left(\left\{x^{\prime}\right\}\right)$.

Proposition 4.1.3. [3, Corollary 4.4]. Let $x$ be an element of a matroid $M$, and $X$ be a subset of $E(M)-x$.

(i) If $x$ is not fixed in $M$, then $x$ is not fixed in $M \backslash X$.

Proposition 4.1.4. [3, Proposition 4.6]. Let $x$ be an element of a matroid $M$.

(i) If $M$ has an element a such that $x$ is fixed in $M \backslash a$, then $x$ is fixed in $M$.

Proposition 4.1.5. [3, Proposition 4.8]. If $x$ and $x^{\prime}$ are independent clones in $M$, then $x$ is fixed in neither $M$ nor $M \backslash x^{\prime}$.

\subsection{Freedom of elements in the union}

The aim of this section is to prove the following theorem. The theorem shows that when taking the union of two matroids, then the elements those two matroids have in common will not be fixed in the union.

Theorem 4.2.1. Let $M_{1}$ and $M_{2}$ be two matroids such that $M=M_{1} \vee M_{2}$, and let $x \in E\left(M_{1}\right) \cap E\left(M_{2}\right)$. If $x$ is not a loop in $M_{1}$ and not a loop in $M_{2}$, then $x$ is not fixed in $M$.

First we establish a lemma.

Lemma 4.2.2. Let $M_{1}$ and $M_{2}$ be two matroids with $\left\{x, x^{\prime}\right\} \subseteq\left[E\left(M_{1}\right) \cap\right.$ $\left.E\left(M_{2}\right)\right]$. If $x$ and $x^{\prime}$ are clones in $M_{1}$ and clones in $M_{2}$, then they are clones in $M_{1} \vee M_{2}$. 
Proof. By Proposition 4.1.2(ii) we need to show that replacing $x$ by $x^{\prime}$ and fixing all other elements is an isomorphism between $\left(M_{1} \vee M_{2}\right) \backslash x$ and $\left(M_{1} \vee M_{2}\right) \backslash x^{\prime}$. To do this it suffices to show that if $I \subseteq E\left(M_{1} \vee M_{2}\right)-\left\{x, x^{\prime}\right\}$, then $I \cup\left\{x^{\prime}\right\}$ is independent in $\left(M_{1} \vee M_{2}\right) \backslash x$ if and only if $I \cup\{x\}$ is independent in $\left(M_{1} \vee M_{2}\right) \backslash x^{\prime}$. By Proposition 2.4.1 $\left(M_{1} \vee M_{2}\right) \backslash x=$ $\left(M_{1} \backslash x\right) \vee\left(M_{2} \backslash x\right)$ and $\left(M_{1} \vee M_{2}\right) \backslash x^{\prime}=\left(M_{1} \backslash x^{\prime}\right) \vee\left(M_{2} \backslash x^{\prime}\right)$. Let us suppose that $I \cup\left\{x^{\prime}\right\}$ is independent in $\left(M_{1} \vee M_{2}\right) \backslash x$, then $I \cup\left\{x^{\prime}\right\}$ is independent in $\left(M_{1} \backslash x\right) \vee\left(M_{2} \backslash x\right)$. It follows by definition of matroid union that there is a partition $(J, K)$ of $I \cup\left\{x^{\prime}\right\}$, where $J$ is independent in $M_{1} \backslash x$ and $K$ is independent in $M_{2} \backslash x$. Without loss of generality, assume that $x^{\prime} \in J$. So that $J=J^{\prime} \cup\left\{x^{\prime}\right\}$. Now since $x$ and $x^{\prime}$ are clones in $M_{1}$ and clones in $M_{2}$, then it follows by Proposition 4.1.2 that we can replace $x$ by $x^{\prime}$ and have $J=J^{\prime} \cup\{x\}$ is independent in $M_{1} \backslash x^{\prime}$ and $K$ is independent in $M_{2} \backslash x^{\prime}$. Hence $\left(J^{\prime} \cup\{x\}\right) \cup K=I \cup\{x\}$ is independent in $\left(M_{1} \backslash x^{\prime}\right) \vee\left(M_{2} \backslash x^{\prime}\right)$. Implying that $I \cup\{x\}$ is independent in $\left(M_{1} \vee M_{1}\right) \backslash x^{\prime}$. Similarly, if $I \cup\{x\}$ is independent in $\left(M_{1} \vee M_{1}\right) \backslash x^{\prime}$, then $I \cup\left\{x^{\prime}\right\}$ is independent in $\left(M_{1} \vee M_{1}\right) \backslash x$. It follows that $x$ and $x^{\prime}$ are indeed clones in $M_{1} \vee M_{2}$.

Proof of Theorem 4.2.1. Assume that $x$ is not a loop in $M_{1}$ and not a loop in $M_{2}$. Let $M_{1}^{\prime}$ be the parallel extension of $M_{1}$ obtained by adding $x^{\prime}$ parallel to $x$, and similarly $M_{2}^{\prime}$ be the parallel extension of $M_{2}$ obtained by adding $x^{\prime}$ parallel to $x$. Now since $x$ and $x^{\prime}$ are parallel to each other in $M_{1}^{\prime}$, and parallel to each other in $M_{2}^{\prime}$, then by Proposition 4.1.1, $x$ and $x^{\prime}$ are clones in $M_{1}^{\prime}$ and clones in $M_{2}^{\prime}$. Therefore by Lemma $4.2 .2, x$ and $x^{\prime}$ are clones in $M_{1}^{\prime} \vee M_{2}^{\prime}$. Now $\left\{x, x^{\prime}\right\}$ is independent in $M_{1}^{\prime} \vee M_{2}^{\prime}$, because it is the union of $\{x\}$ and $\left\{x^{\prime}\right\}$, where $\{x\}$ is independent in $M_{1}^{\prime}$ and $\left\{x^{\prime}\right\}$ is independent in $M_{2}^{\prime}$. Meaning $\{x\}$ and $\left\{x^{\prime}\right\}$ are independent clones in $M_{1}^{\prime} \vee M_{2}^{\prime}$. By Proposition 4.1.5 $x$ is not fixed in $M_{1}^{\prime} \vee M_{2}^{\prime}$. By Proposition 4.1.3(i), $x$ is not fixed in $\left(M_{1}^{\prime} \vee M_{2}^{\prime}\right) \backslash x^{\prime}$. But by Proposition 2.4.1 $\left(M_{1}^{\prime} \vee M_{2}^{\prime}\right) \backslash x^{\prime}=$ $\left(M_{1}^{\prime} \backslash x^{\prime}\right) \vee\left(M_{2}^{\prime} \backslash x^{\prime}\right)=M_{1} \vee M_{2}$. Therefore $x$ is not fixed in $M_{1} \vee M_{2}$ as required.

As a consequence of Theorem 4.2.1, we get the following result.

Lemma 4.2.3. If every element in a connected matroid $M$ is fixed, then $M$ is indecomposable. 
Proof. Suppose $M$ is decomposable. By Proposition 2.2.2 we can assume that $M$ is the union of two matroids $M_{1}$ and $M_{2}$, such that $M_{1}$ and $M_{2}$ are loopless. If $x \in E\left(M_{1}\right) \cap E\left(M_{2}\right)$, then it follows from Theorem 4.2.1 that $x$ must be non-fixed in $M$. But every element in $M$ is fixed. Therefore there are no elements in the intersection of $E\left(M_{1}\right)$ and $E\left(M_{2}\right)$. So that $M$ is disconnected, contradicting the fact that $M$ is connected. Hence $M$ is indecomposable.

\subsection{Freedom of elements in binary and ternary matroids}

In this section we look at which elements in binary and ternary matroids are fixed and not fixed. First we look at binary matroids.

Lemma 4.3.1. [2, Lemma 5.6] Let $M$ be a connected binary matroid having at least two elements and let $x$ be an element of $M$. If $M \backslash x$ is connected, then $x$ is fixed in $M$.

As a result of combining Lemma 4.3.1, Proposition 1.8.16 and Lemma 1.8.18 we get the following.

Corollary 4.3.2. With the exception of $U_{2,3}$, every element of a 3-connected binary matroid $M$ is fixed.

Proof. Suppose that $M$ has a non-fixed element $a$. Then $M \backslash a$ is disconnected by Lemma 4.3.1. But that by Proposition 1.8 .16 contradicts the fact that $M$ is 3-connected. By Lemma 1.8.18, $U_{2,3}$ is an exception to this. Therefore every element of a 3-connected binary matroid is fixed, except $U_{2,3}$.

The following theorem provides a link between binary and 3-connected ternary matroids using non-fixed elements in ternary matroids.

Theorem 4.3.3. Let $M$ be a 3-connected ternary matroid. If $x \in E(M)$ and $x$ is not fixed, then $M \backslash x$ is a regular matroid.

Before we prove Theorem 4.3.3 we need definitions. A well-closed class of matroids is a class of matroids that is closed under isomorphism, minors, 
and duality. A 3-connected matroid $N$ in a well-closed class $\mathcal{N}$ is a universal stabilizer for $\mathcal{N}$ if the following holds for all 3-connected matroids $M$ in $\mathcal{N}$ and all $x$ in $E(M):$ if $M \backslash x$ is 3 -connected and has an $N$-minor, then $x$ is fixed in $M$, and if $M / x$ is 3 -connected and has an $N$-minor, then $x$ is cofixed in $M$. For more details on universal stabilizers see [2] and [3]. The next theorem will be used in the proof.

Theorem 4.3.4. [2, Theorem 5.3] Let $N$ be a 3-connected matroid in the well-closed class $\mathcal{N}$. Then $N$ is a universal stabilizer for $\mathcal{N}$ if and only if the following condition holds for every 3-connected matroid $M$ in $\mathcal{N}$ and every $x$ in $E(M)$ :

If $M \backslash x$ is connected with an $N$-minor, then $x$ is fixed in $M$, and if $M / x$ is connected with an $N$-minor, then $x$ is cofixed in $M$.

Proof of Theorem 4.3.3. Suppose $M \backslash x$ is not binary, then by Theorem 1.7.5 it must have a $U_{2,4}$-minor. It is shown in [3, Section 2] that $U_{2,4}$ is a universal stabilizer for the class of all ternary matroids. Now if $M \backslash x$ has a $U_{2,4}$-minor, then it follows that $M$ has a $U_{2,4}$-minor. Since $M$ is 3 -connected, and given that $x$ is not fixed in $M$, then it follows by Theorem 4.3.4 that $M \backslash x$ is disconnected. By Lemma 1.8.18, the only case where this would happen is when $M \cong U_{2,3}$. But in that case $M$ would not have a $U_{2,4}$-minor. So we have shown that if $M$ has a $U_{2,4^{-}}$minor, then it does not have a $U_{2,4^{-}}$ minor, contradiction. Then it must be true that $M \backslash x$ is binary. Moreover, by Proposition 1.7.2 $M \backslash x$ is also ternary. Meaning $M \backslash x$ is binary and ternary. Therefore by Theorem 1.7.8, $M \backslash x$ is regular.

The following corollary shows that by deleting two non-fixed elements from a ternary matroid, it gets disconnected.

Corollary 4.3.5. Let $M$ be a 3-connected ternary matroid not isomorphic to $U_{2,3}$. Then for all $x$ and $y$ in $E(M)$ where $x$ and $y$ are not fixed in $M$, $M \backslash x, y$ is a disconnected matroid.

Proof. Since $M$ is 3-connected, then by Proposition 1.8.16 $M \backslash x$ is connected. Since $x$ is not fixed, then by Theorem 4.3.3 $M \backslash x$ is a binary matroid. By Proposition 4.1.3 $y$ is not fixed in $M \backslash x$. By Corollary 4.3.2 every element in a 3 -connected binary matroid is fixed. So that $M \backslash x$ cannot 
be 3-connected. Therefore $M \backslash x$ is a binary connected but not 3-connected matroid containing the non-fixed element $y$. It follows by Lemma 4.3.1 that $M \backslash x, y$ is disconnected.

\subsection{Decomposable binary matroids}

\subsubsection{Alternative proof of Cunningham's theorem}

In this section we give an alternative proof for Cunningham's Theorem, which we also restate for convenience. We also restate and give an alternative proof for Corollary 2.3.16, and give an example showing that matroid union is not closed under duality.

Theorem 4.4.1. [1] $A$ binary matroid $M$ on $E$ is indecomposable if and only if $M$ is connected and $M \backslash e$ is connected for each $e \in E$.

Proof. Let $M$ be a connected binary matroid. If $M$ is decomposable, then by Lemma 4.2 .3 it must have at least one non-fixed element $x$. Consider $M \backslash x$. By Lemma 4.3.1 $M \backslash x$ is disconnected. So if $M$ is decomposable, then it contains at least one element $x$ such that $M \backslash x$ is disconnected. Conversely, if $M$ contains an element $x$ such that $M \backslash x$ is disconnected, then it has a separation $(A, B)$. By Theorem 1.8.6(i) $M=S(M / A, M / B)$. It follows then by Proposition 3.2.1 that $M=(M / A) \vee(M / B)$. But since $M=S(M / A, M / B)$, then both $M / A$ and $M / B$ must have rank strictly greater than zero. Hence $M$ is decomposable.

Next we restate and re-prove Corollary 2.3.16.

Corollary 4.4.2. With the exception of $U_{2,3}$, every 3-connected binary matroid is indecomposable.

Proof. Suppose $M$ is 3-connected and is binary. Then by Corollary 4.3.2, every element in $M$ is fixed. So that by Lemma $4.2 .3, M$ is indecomposable. Since every uniform matroid is decomposable by corollary 2.3 .14 , then the only exception to this case is $U_{2,3}$.

We can now give a restatement of Cunningham's theorem using the notion of non-fixed elements. 
Theorem 4.4.3. Let $M$ be a connected binary matroid. $M$ is decomposable if and only if it contains at least one non-fixed element $x$. Moreover, if $x$ is not fixed, then $M \backslash x$ is not connected, so that there is a separation $(A, B)$ in $M \backslash x$. In this case $M=(M / A) \vee(M / B)$.

Next we illustrate an application of Lemma 4.2.3. We will show that if $M$ is decomposable, then $M^{*}$ is not necessarily also decomposable. Consider the following example.

Example 4.4.4. Consider the two matroids $M_{1}$ and $M_{2}$ where $M_{1} \cong M_{2} \cong$ $F_{7}^{*} . E\left(M_{1}\right)=\{a, b, c, d, e, f, h\}, E\left(M_{2}\right)=\{h, i, j, k, l, m, g\} . S\left(M_{1}, M_{2}\right)$ on the base-point $h$ is by Proposition 3.2.1 decomposable. The dual of that, however, is $P\left(M_{1}^{*}, M_{2}^{*}\right)$, where $M_{1}^{*} \cong M_{2}^{*} \cong F_{7}$. Every element in this parallel connection is fixed as shown in Figure 4.1. Which by Lemma 4.2.3 means it is indecomposable.

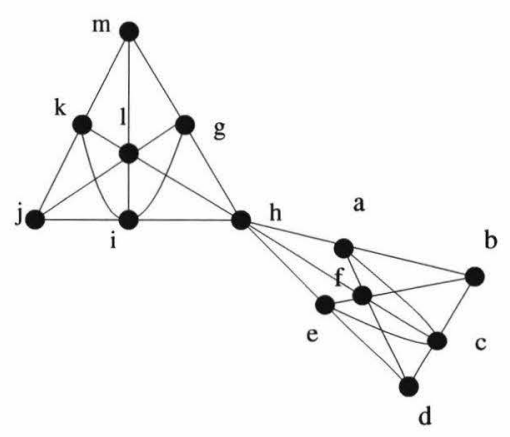

Figure 4.1: A parallel connection of two Fano matroids. 


\section{Chapter 5}

\section{Decomposable ternary matroids}

In this chapter we show what essential property every decomposable 3connected ternary matroid must have. We also present a theorem showing how a 3-connected ternary matroid can be composed from two other matroids, and also present a partial converse of that. From now on we will be assuming that our matroid union is loopless. First we start by identifying the ternary matroids that for certain are indecomposable, as shown in the next section.

\subsection{4-connected ternary matroids are indecompos- able}

The main result of this section is the following:

Lemma 5.1.1. With the exception of $U_{2,3}$, 4-connected ternary matroids are indecomposable.

First we need a couple of results.

Proposition 5.1.2. If $M$ is a 4-connected ternary matroid, then with the exception of $U_{2,3}, M$ has at most one non-fixed element.

Proof. Suppose that $M$ has two non-fixed elements $x$ and $y$. Then by Corollary 4.3.5 $M \backslash x, y$ is disconnected. But $M$ is 4 -connected, and hence by 
Proposition 1.8.16 cannot be disconnected by deleting two elements, so we get a contradiction. $U_{2,3}$ is the exception here. Therefore with the exception of $U_{2,3}, M$ has at most one non-fixed element.

Proposition 5.1.3. Let $M_{1}$ and $M_{2}$ be two loopless matroids such that $M=M_{1} \vee M_{2}$. If $S$ is the set of non-fixed elements of $M$, then $E\left(M_{1}\right) \cap$ $E\left(M_{2}\right) \subseteq S$.

Proof. If $x \in E\left(M_{1}\right) \cap E\left(M_{2}\right)$, then by Theorem $4.2 .1 x$ is not fixed in $M$. Therefore $x \in S$, so that $E\left(M_{1}\right) \cap E\left(M_{2}\right) \subseteq S$.

Now we prove Lemma 5.1.1.

Proof of Lemma 5.1.1. Suppose $M$ is decomposable. Then by Proposition 2.2.2 there exist loopless matroids $M_{1}$ and $M_{2}$ such that $M=M_{1} \vee M_{2}$. If $M$ has every element fixed, then by Theorem 4.2.3 $M$ is indecomposable. So assume it has some non-fixed elements. But if $M$ has some non-fixed elements, then by Proposition 5.1.2 it has at most one non-fixed element, call this element $a$. It follows by Proposition 5.1.3 that $E\left(M_{1}\right) \cap E\left(M_{2}\right)=$ $\{a\}$. By Proposition 3.2.1 $M=S\left(M_{1}, M_{2}\right)$. So that $M$ has a 2-separation, meaning it is not 4-connected.

\subsection{Property of decomposability}

The aim of this section is to prove the following theorem, which shows what essential property every decomposable 3-connected ternary matroid has.

Theorem 5.2.1. Let $M$ be a loopless 3-connected ternary matroid not isomorphic to $U_{2,3}$. If $M$ is decomposable, then it contains at least two non-fixed elements $p$ and $q$.

Proof. If $M$ is 4-connected, then by Lemma 5.1 .1 it is indecomposable. So assume that $M$ is not 4-connected. If $M$ is decomposable, then since it is loopless, we can assume by Proposition 2.2.2 that $M=N_{1} \vee N_{2}$, where both $N_{1}$ and $N_{2}$ are loopless. Since $M$ is connected, then $N_{1}$ and $N_{2}$ cannot be disjoint. If $N_{1}$ and $N_{2}$ have only one element in common, then by Proposition 3.2.1 $M$ is a series connection, and hence not 3-connected, giving a 
contradiction. So $N_{1}$ and $N_{2}$ must have at least two elements in common, call them $p$ and $q$. Since $p$ and $q$ are not loops, then by Theorem 4.2.1, $p$ and $q$ are not fixed in $M$. So if $M$ is decomposable, then it contains at least two non-fixed elements.

\subsection{Composing ternary matroids}

The aim of this section is to prove the following theorem which shows how a ternary matroid can be constructed from two other matroids. Recall that if $M=M_{1} \vee M_{2}$ is disconnected, then $M=N_{1} \oplus N_{2}$, for some disjoint matroids $N_{1}$ and $N_{2}$. If $M=M_{1} \vee M_{2}$ is 2-connected, then $\mid E\left(M_{1}\right) \cap$ $E\left(M_{2}\right) \mid \geq 1$, if $M=M_{1} \vee M_{2}$ is 3-connected, then $\left|E\left(M_{1}\right) \cap E\left(M_{2}\right)\right| \geq 2$, and if $M=M_{1} \vee M_{2}$ is 4-connected, then $\left|E\left(M_{1}\right) \cap E\left(M_{2}\right)\right| \geq 3$. Due to the fact that 4-connected ternary matroids are indecomposable as shown in Section 5.1, we are not interested in ternary matroids that are 4-connected. For this reason, we shall focus on the union of two matroids that have exactly two elements in common, thus making sure the union is not 4-connected. Note also that having two elements in common, $x$ and $y$, means that $x$ and $y$ are non-fixed in the union by Theorem 4.2.1. Thus the 3-connected decomposable ternary matroid meets the decomposition property mentioned in Theorem 5.2.1.

Theorem 5.3.1. Let $M_{1}$ and $M_{2}$ be two loopless regular matroids with $\left|E\left(M_{1}\right) \cap E\left(M_{2}\right)\right| \leq 2$, such that $M_{1} \vee M_{2}$ is 3-connected. Then $M=$ $M_{1} \vee M_{2}$ is a ternary matroid.

We first establish some lemmas.

Lemma 5.3.2. Let $M$ be a matroid such that $M=M_{1} \vee M_{2}$ where $M_{1}$ and $M_{2}$ are both loopless binary matroids with $\left|E\left(M_{1}\right) \cap E\left(M_{2}\right)\right|=1$, then $M$ is a binary matroid.

Proof. Since $\left|E\left(M_{1}\right) \cap E\left(M_{2}\right)\right|=1$, then by Proposition 3.2.1 $M_{1} \vee M_{2}=$ $S\left(M_{1}, M_{2}\right)$. But since $M_{1}$ and $M_{2}$ are both binary, then it follows by Proposition 1.8 .14 that $S\left(M_{1}, M_{2}\right)=M$ is also binary. 
Lemma 5.3.3. Let $M$ be a matroid such that $M=M_{1} \vee M_{2}$ where $M_{1}$ and $M_{2}$ are both loopless binary matroids with $E\left(M_{1}\right) \cap E\left(M_{2}\right)=\{x, y\}$. Then $M \backslash x$ and $M \backslash y$ are both binary matroids.

Proof. Without loss of generality, consider $M \backslash x$. By Proposition 2.4.1 $M \backslash x=\left(M_{1} \vee M_{2}\right) \backslash x=\left(M_{1} \backslash x\right) \vee\left(M_{2} \backslash x\right) .\left(M_{1} \backslash x\right)$ and $\left(M_{2} \backslash x\right)$ have only one element in common which is $y$. Moreover, $\left(M_{1} \backslash x\right)$ and $\left(M_{2} \backslash x\right)$ are both binary by Proposition 1.7.2. So by Lemma 5.3.2 $M \backslash x$ is binary. Similarly, $M \backslash y$ is also binary.

Lemma 5.3.4. Let $M$ be a matroid and let $x \in E(M)$. If $M / x \cong U_{2,5}$, then for all $z \in E(M)-\{x\}, M \backslash z$ is non-binary.

Proof. Since $M / x \cong U_{2,5}$, then $M / x \backslash z \cong U_{2,4}$. By Proposition 1.4.2(iii) $M / x \backslash z=M \backslash z / x$. Hence $M \backslash z$ has a $U_{2,4}$-minor, so that $M \backslash z$ is nonbinary.

Lemma 5.3.5. Let $M$ be a matroid and let $x \in E(M)$. If $M \backslash x \cong U_{2,5}$, then for all $z \in E(M)-\{x\}, M \backslash z$ is non-binary.

Proof. Since $M \backslash x \cong U_{2,5}$, then $M \backslash x, z \cong U_{2,4}$. By Proposition 1.4.2(i) $M \backslash x, z=M \backslash z, x$. Meaning $M \backslash z$ has a $U_{2,4}$-minor, and hence nonbinary.

The following proposition will also be used in proving Theorem 5.3.1.

Proposition 5.3.6. [5, Corollary 11.2.20] A 3-connected matroid whose rank and corank exceed two has a minor isomorphic to one of $M\left(\mathcal{W}_{3}\right), \mathcal{W}^{3}$, $Q_{6}, P_{6}$ and $U_{3,6}$.

First we prove a lemma that shows how to construct a matroid that has no $U_{2,5}$ or $U_{3,5}$-minors.

Lemma 5.3.7. Suppose that $M_{1}$ and $M_{2}$ are both loopless binary matroids with $\left|E\left(M_{1}\right) \cap E\left(M_{2}\right)\right| \leq 2$, such that $M_{1} \vee M_{2}$ is 3-connected. Then $M=$ $M_{1} \vee M_{2}$ has no $U_{2,5}$ or $U_{3,5}$-minors.

Proof. The proof is by induction. First we show the theorem holds for matroids with $|E(M)| \leq 7$, and that becomes our base case. We then show 
that it is not possible for $M$ to have a $U_{2,5}$ or $U_{3,5}$-minors when $|E(M)| \geq 8$. But first we show that $E\left(M_{1}\right)$ and $E\left(M_{2}\right)$ cannot have less that two elements in common.

If $\left|E\left(M_{1}\right) \cap E\left(M_{2}\right)\right|=0$, then $M$ is disconnected, giving a contradiction. If $\left|E\left(M_{1}\right) \cap E\left(M_{2}\right)\right|=1$, then $M$ is a series connection and hence not 3connected, giving a contradiction. Therefore we may assume from now on that $\left|E\left(M_{1}\right) \cap E\left(M_{2}\right)\right|=2$.

Base case step. Case 1. Suppose that $|E(M)|=5$. We break this into two further cases, one for each minor.

Case 1.1. Suppose $M$ has a $U_{2,5}$-minor, then since $|E(M)|=5$, then $M \cong U_{2,5}$. Now we examine the two different possibilities in which $M$ can be constructed from two matroids with two elements in common.

Suppose that $M$ is the union of $M_{1}$ and $M_{2}$, where $E\left(M_{1}\right)$ has 5 elements, $E\left(M_{2}\right)$ has 2 elements, with two elements in common as shown in Figure 5.1. Let $E(M)=\{a, b, c, x, y\}, E\left(M_{1}\right)=\{a, b, c, x, y\}$ and $E\left(M_{2}\right)=\{x, y\}$. Since any 2-element set in $U_{2,5}$ is independent, and because $a$ and $b$ are only in $E\left(M_{1}\right)$, then $\{a, b\}$ is independent in $M_{1}$. Also, since both $M_{1}$ and $M_{2}$ are loopless, then any 1-element set in $M_{2}$ is independent. So $\{x\}$ is independent in $M_{2}$. But then by matroid union definition, we should have $\{a, b\} \cup\{x\}=\{a, b, x\}$ is independent in $M$. Contradicting the fact that $M \cong U_{2,5}$ is a rank-2 matroid. Therefore it is not possible for $M$ to have a $U_{2,5}$-minor when $E\left(M_{1}\right)$ has 5 elements, and $E\left(M_{2}\right)$ has 2 elements, with two elements in common.

Now suppose that $M$ is the union of $M_{1}$ and $M_{2}$, where $E\left(M_{1}\right)$ has 4 elements, and $E\left(M_{2}\right)$ has 3 elements, with two elements in common as shown in Figure 5.2. Let $E(M)=\{a, b, c, x, y\}, E\left(M_{1}\right)=\{b, c, x, y\}$, and $E\left(M_{2}\right)=$ $\{x, y, a\} .\{b, c\}$ is independent in $M$. So $\{b, c\}$ must be independent in $M_{1}$. Since $M_{2}$ is loopless, then $\{a\}$ must be independent in $M_{2}$. Therefore $\{b, c\} \cup\{a\}=\{a, b, c\}$ must be independent in $M$, contradicting the fact that $M$ is a rank-2 matroid. So $M$ cannot have a $U_{2,5}$-minor when $|E(M)|$ $=5$.

Case 1.2. Suppose $M$ has a $U_{3,5}$-minor, then since $|E(M)|=5$, then $M \cong U_{3,5}$. We will examine the two different possibilities in which $M$ can be constructed from two matroids with two elements in common. 


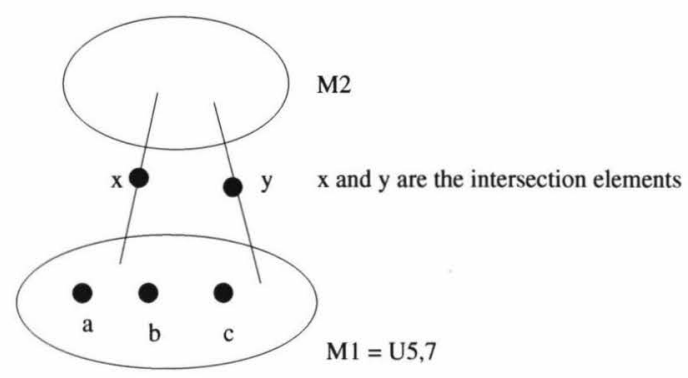

Figure 5.1: $E\left(M_{1}\right)$ has 5 elements and $E\left(M_{2}\right)$ has 2 elements.

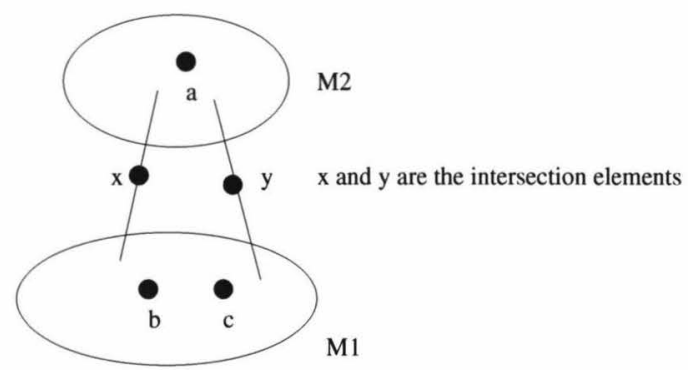

Figure 5.2: $E\left(M_{1}\right)$ has 4 elements and $E\left(M_{2}\right)$ has 3 elements.

Suppose that $M$ is the union of $M_{1}$ and $M_{2}$, where $E\left(M_{1}\right)$ has 5 elements, and $E\left(M_{2}\right)$ has 2 elements, with two elements in common as shown in Figure 5.1. Let $E(M)=\{a, b, c, x, y\}, E\left(M_{1}\right)=\{a, b, c, x, y\}$ and $E\left(M_{2}\right)=\{x, y\}$. Since any 3 -element set in $U_{3,5}$ is independent, and because $a, b$ and $c$ are only in $E\left(M_{1}\right)$, then it must that $\{a, b, c\}$ is independent in $M_{1}$. Element $x$ is not a loop in $M_{2}$, so $\{x\}$ is independent in $M_{2}$. It follows that $\{a, b, c\} \cup\{x\}=$ $\{a, b, c, x\}$ is independent in $M$, contradicting the fact that $M \cong U_{3,5}$ is a rank-3 matroid.

Now suppose that $M$ is the union of $M_{1}$ and $M_{2}$ where $M_{1}$ has 4 elements, $M_{2}$ has 3 elements, with two elements in common as shown in Figure 5.2. Let $E(M)=\{a, b, c, x, y\}, E\left(M_{1}\right)=\{b, c, x, y\}$ and $E\left(M_{2}\right)=\{x, y, a\}$. If one of the two matroids is rank- 0 , then $M$ is indecomposable, giving a contradiction. So one of the two matroids must be rank-1, and the other must be rank-2. Since any two-element set in $M$ is independent, then $\{b, c\}$ must be independent in $M$. So that $\{b, c\}$ must be independent in $M_{1}$. 
Therefore $M_{1}$ is the rank- 2 matroid, and $M_{2}$ is the rank-1 matroid. It follows that any 2 -element set in $M_{2}$ is a circuit. Since $\{a, x, b\}$ is independent in $M$, it is the union of two independent sets. But $\{a, x\}$ is a circuit in $M_{2}$. Therefore it must be that $\{b, x\}$ is independent in $M_{1}$. Similarly, the fact that $\{a, x, c\}$ is independent in $M$, implies that $\{c, x\}$ must be independent in $M_{1}$. Also since the elements $b$ and $c$ are only in $E\left(M_{1}\right)$, then $\{b, c\}$ must be independent in $M_{1}$ as well. In fact by following this reasoning we can show that any 2-element set in $M_{1}$ is independent. But $M_{1}$ is rank-2, meaning any set with size 3 is a circuit. Since $M_{1}$ has four elements, then it follows that $M_{1} \cong U_{2,4}$. This contradicts the fact that $M_{1}$ is binary. So we conclude that when $|E(M)|=5, M$ has no $U_{2,5}$ or $U_{3,5}$-minors. Note that this case does not require the fact that the union is 3 -connected.

Case 2. Suppose that $|E(M)|=6$. We break this into two further cases, one for each minor.

Case 2.1. Suppose $M$ has a $U_{2,5}$-minor. Since $|E(M)|=6$, there are two different ways we can obtain a $U_{2,5}$-minor from $M$.

The first one is when $M \backslash p \cong U_{2,5}$ for some $p \in E(M)$. By Lemma 5.3.5, $M \backslash p \cong U_{2,5}$ implies that $M \backslash z$ is non-binary for all $z \in E(M)-\{p\}$. But $z$ can be an intersection element of $E\left(M_{1}\right)$ and $E\left(M_{2}\right)$. So that by deleting $z$ from $M$, we get by Lemma 5.3 .3 a binary matroid, giving a contradiction. Therefore $U_{2,5}$ cannot be obtained by deleting an element from $M$.

The second possibility is when $M / p \cong U_{2,5}$. By Lemma 5.3.4, $M / p \cong$ $U_{2,5}$ implies that $M \backslash z$ is non-binary for all $z \in E(M)-\{p\}$. If $z$ is an intersection element of $E\left(M_{1}\right)$ and $E\left(M_{2}\right)$, then by Lemma 5.3.3 $M \backslash z$ is a binary matroid. So we get a contradiction. Therefore it is not possible to obtain $U_{2,5}$ from $M$ by contracting or deleting an element.

Case 2.2. Suppose $M$ has a $U_{3,5}$-minor, then since $|E(M)|=6$, there are two different ways we can obtain a $U_{3,5}$-minor from $M$.

The first one is when $M \backslash p \cong U_{3,5}$ for some $p \in E(M)$. If $p$ is not in $E\left(M_{1}\right) \cap E\left(M_{2}\right)$, then suppose without loss of generality that $p \in E\left(M_{1}\right)$. By Proposition 2.4.1 $M \backslash p=\left(M_{1} \backslash p\right) \vee M_{2}$. In this case $M \backslash p$ is a 5element decomposable matroid that is the union of two binary matroids with two elements in common. It follows by Case 1.2 that we cannot have $M \backslash p \cong U_{3,5}$. If on the other hand $p$ was in the intersection, then deleting 
$p$ from $M$ leaves $E\left(M_{1}\right)$ and $E\left(M_{2}\right)$ with only one element in common. So $M \backslash p$ by Proposition 3.2.1 is a series connection of two matroids. But $U_{3,5}$ is not a series connection of two matroids, so this contradicts the fact that $M \backslash p \cong U_{3,5}$.

The second possibility is when $M / p \cong U_{3,5}$. If $p$ is not in $E\left(M_{1}\right) \cap$ $E\left(M_{2}\right)$, then we lose no generality if we assume that $p \in E\left(M_{1}\right)$. By Proposition 2.4.5 $M / p=M_{1} / p \vee M_{2}$. But $M / p$ is a 5-element decomposable matroid that is the union of two binary matroids that have two elements in common. So we arrive at the same contradiction shown in Case 1.2. If $p$ is in the intersection, then let us consider the dual of $M / p$, to see what possibilities there are for $M$. Since $M / p \cong U_{3,5}$, then $M^{*} \backslash p \cong U_{2,5}$, and we have the following possibilities:

(i) Either $p$ is a coloop in $M^{*}$, implying that $p$ is a loop in $M$, contradicting the fact that $M$ is connected.

(ii) $p$ is a loop in $M^{*}$, implying that $p$ is a coloop in $M$, contradicting the fact that $M$ is connected.

(iii) $M^{*}$ has $p$ in a parallel class with another element, implying that $M$ has $p$ in a series pair with another element. But $M \backslash p$ must be connected by Proposition 1.8.16, giving a contradiction.

(iv) Or $M^{*} \cong U_{2,6}$, implying that $M \cong U_{4,6}$. Choose an element $z$ not in $E\left(M_{1}\right) \cap E\left(M_{2}\right)$. Assume without loss of generality that $z \in E\left(M_{1}\right)$. Then

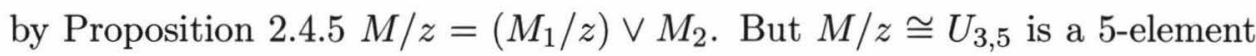
decomposable matroid that is the union of two binary matroids with two elements in common, contradicting what was shown in Case 1.2.

Case 3. Suppose that $|E(M)|=7$. We break this into two further cases, one for each minor.

Case 3.1. Suppose $M$ has a $U_{2,5}$-minor, then since $|E(M)|=7$, there are three different ways we can obtain a $U_{2,5}$-minor from $M$. First one is deleting two elements from $M$, second one is deleting one element and contracting another, and the third one is contracting two elements from $M$.

Consider the two ways involving a deletion. Suppose that either $M \backslash$ $p, q \cong U_{2,5}, M \backslash p / q \cong U_{2,5}$, or $M \backslash q / p \cong U_{2,5}$. If the deletion element, say $p$, is in the intersection, then $M \backslash p$ is the union of two binary matroids that have one element in common. It follows by Proposition 3.2.1 that $M \backslash p$ is 
a series connection of two binary matroids. So that by Proposition 1.8.14 $M \backslash p$ is a binary matroid too. Hence $M \backslash p$ does not have a $U_{2,5}$-minor, contradicting the assumption. If none of the deletion elements are in the intersection, then we can delete one of them say $p$, and get by Proposition 2.4.1 $M \backslash p$ as a 6-element decomposable matroid. But $M \backslash p$ is the union of two binary matroids that have two elements in common, and has a $U_{2,5^{-}}$ minor, which contradicts what was shown in Case 2.1.

Now consider $M / p / q \cong U_{2,5}$. If only one of the elements $p$ and $q$ is in the intersection, say $q$, then by Proposition $2.4 .5 \mathrm{M} / \mathrm{p}$ is a 6 -element decomposable matroid. It is the union of two binary matroids that have two elements in common, and it has a $U_{2,5}$-minor. But that contradicts Case 2.1. Similarly, if none of the elements $p$ and $q$ are in the intersection, then $M / p$ and $M / q$ also give the same contradiction. Suppose on the other hand that both $p$ and $q$ are in the intersection. Let $N=M / p$, so that $N / q \cong U_{2,5}$. It follows that $N^{*} \backslash q \cong U_{3,5}$. Let us now consider the possibilities for what $N$ could be:

(i) If $q$ is a coloop in $N^{*}$, then it implies that $N^{*}$ is disconnected, and hence by Proposition 1.5.4 $N$ is disconnected. But that contradicts the fact that $M$ is 3-connected by Proposition 1.8.16.

(ii) $q$ is a loop in $N^{*}$, implying that $q$ is a coloop in $N$ meaning $N$ is disconnected, contradicting Proposition 1.8.16.

(iii) $q$ is in parallel to another element in $N^{*}$, implying that $N$ has a series pair. But if $N=M / p$ has a series pair, then $M$ must have a series pair too, which means $M$ can be disconnected by removing one element, contradicting the fact that it is 3-connected.

Having made the observations (i-iii), we see that element $q$ must be on the same plane as the other elements of $U_{3,5}$. So that $N^{*}$ must be rank-3. Moreover, $q$ can be collinear with at most two elements in $N^{*}$, meaning $q$ cannot create a 2 -separation in $N^{*}$. So that $N^{*}$ is 3 -connected. But $N^{*}$ has 6 elements, meaning its corank exceeds two. So it follows from Corollary 5.3.6 that there are only 5 possibilities for $N^{*}$, namely $M\left(\mathcal{W}_{3}\right), \mathcal{W}^{3}, Q_{6}, P_{6}$ and $U_{3,6}$ as shown in Figure 5.3. From those 5 matroids, however, only the ones shown in Figure $5.3\left(\mathrm{a}, \mathrm{b}\right.$ and c) have $N^{*} \backslash q \cong U_{3,5}$. If we examine their duals as shown in Figure 5.3(d, e and $\mathrm{f})$ respectively, we will find that 
no matter where the intersection element $q$ is located in $N, N \backslash q$ always has a $U_{2,4}$-minor. Hence $N \backslash q$ is not binary. If $N \backslash q$ is non-binary, then $M / p \backslash q=M \backslash q / p$ is non-binary. But by Lemma 5.3.3 $M \backslash q$ is supposed to be binary, contradiction. Therefore when $|E(M)|=7, M$ has no $U_{2,5}$-minor.

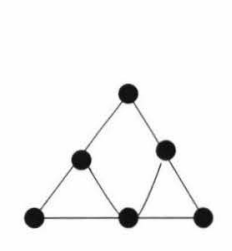

$\mathrm{M}\left(\mathrm{W}_{3}\right)$

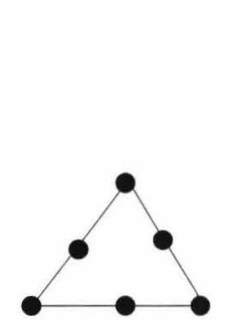

$\mathrm{W}^{3}$

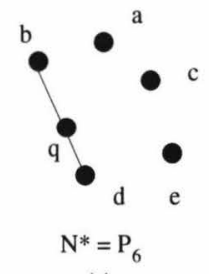

(a)

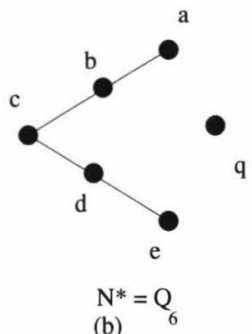

(b)

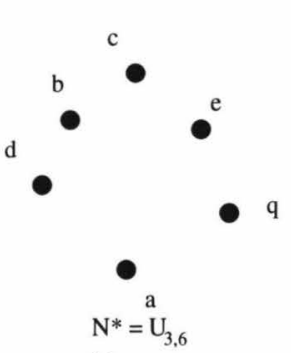

(c)

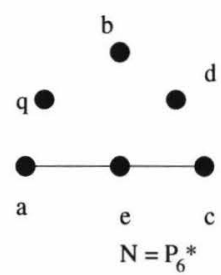

(d)

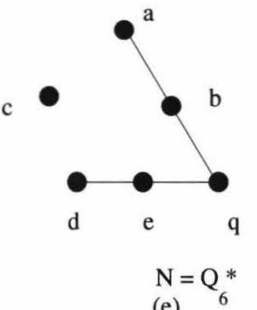

(e)

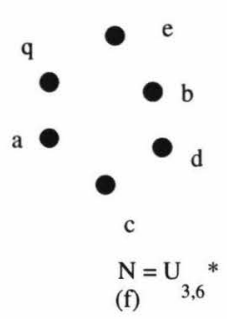

Figure 5.3: Matroid with $U_{3,5}$-minor.

Case 3.2. Suppose $M$ has a $U_{3,5}$-minor, since $|E(M)|=7$, then we consider the different ways $M$ can have a $U_{3,5}$-minor.

The two ways involving deletions are $M \backslash p, q \cong U_{3,5}, M \backslash p / q \cong U_{3,5}$ or $M \backslash q / p \cong U_{3,5}$. If none of the elements $p$ and $q$ are in the intersection, then $M \backslash p$ having a $U_{3,5}$-minor contradicts what was shown in Case 2.1. If any of the deletion elements is in the intersection, say $p$, then $M \backslash p$ is a series connection of two binary matroids. Hence by Proposition 1.8.14 is binary, contradicting the fact that $M \backslash p$ has a $U_{3,5}$-minor. 
The third way is when $M / p / q \cong U_{3,5}$. Suppose none of the two elements are in the intersection. Then we can contract one element, say $p$, and get by Proposition 2.4.5 $\mathrm{M} / \mathrm{p}$ as a 6-element decomposable matroid, constructed from the union of two binary matroids with two elements in common, and it must be 3-connected. That contradicts what was shown in Case 2.2. Suppose one of the elements, say $p$, is in the intersection and $q$ is not. Then $M / q$ is a 6 -element decomposable matroid, constructed from the union of two binary matroids with two elements in common, which also must be 3connected, again contradicting what was shown in Case 2.2. If on the other hand both $p$ and $q$ are in the intersection, then since $M / p / q \cong U_{3,5}$, it follows that $M^{*} \backslash p \backslash q \cong U_{2,5}$. Now we look at the different possibilities for $M^{*}$.

$M^{*}$ is a 5-point line in addition to the two other elements $p$ and $q$. Because $p$ and $q$ are intersection elements, then by Theorem 4.2.1 they are not fixed in $M$.

(i) If either $p$ or $q$ is a loop or coloop in $M^{*}$, then it is a coloop or loop in $M$ respectively, leading to contradictions as was shown in Case 3.1.

(ii) If either $p$ or $q$ is in parallel with any other element $z$ in $M^{*}$, then that makes $\{p, z\}$ or $\{q, z\}$ a series pair in $M$. But then it follows that $M$ can be disconnected by deleting one of the series pair elements, contradicting the fact that $M$ is 3 -connected. We would get the same contradiction if $p$ and $q$ are parallel to each other.

(iii) If $p$ or $q$ is in a series pair with any other element $z$ in $M^{*}$, then that makes $\{p, z\}$ or $\{q, z\}$ a parallel pair in $M$ respectively, and hence fixing $p$ or $q$ in $M$. Thereby contradicting the fact that $p$ and $q$ are both non-fixed in $M$. The same contradiction follows if $p$ and $q$ make a series pair together.

Therefore the only place left for $p$ and $q$ in $M^{*}$ is on the 5-point line, such that $\{p, q\}$ is independent on the line. Thus making $M^{*} \cong U_{2,7}$. Hence $M \cong U_{5,7}$. Choose $z \in E(M)$, such that $z$ is not an intersection element. Assume without loss of generality that $z \in E\left(M_{1}\right)$. Since $\left(M_{1} / z\right)$ is binary by Proposition 1.7.2, and $M_{2}$ is binary by assumption. Then it follows that $M / z=\left(M_{1} / z\right) \vee M_{2} \cong U_{4,6}$ is a 6-element decomposable matroid made from the union of two binary matroids. Moreover, the two matroids still have two elements in common, $M / z$ has a $U_{3,5}$-minor, and must be 3 -connected as well. This contradicts what was shown in Case 2.2. Therefore we conclude 
that when $|E(M)|=7, M$ has no $U_{3,5}$-minor. Thus we have established a base case for our induction.

Inductive step. Assume that $|E(M)|=\left|E\left(M_{1}\right) \cup E\left(M_{2}\right)\right| \geq 8$. Also assume that the result holds whenever $|E(M)| \leq 7$, for all pairs of binary matroids $M_{1}$ and $M_{2}$ such that $E\left(M_{1}\right) \cap E\left(M_{2}\right)=\{x, y\}$. Suppose the result fails for $M$. Then there exists $X, Y \subseteq E(M)$, such that $\left(M_{1} \vee M_{2}\right) \backslash X / Y \cong$ $U_{2,5}$ or $U_{3,5}$. Since $|E(M)| \geq 8$, then there exists $z$ in $(X \cup Y)-\{x, y\}$. Without loss of generality, assume $z$ is in $E\left(M_{1}\right)$, and also assume that $z$ is in $X$. Then by Proposition 2.4.1 $M \backslash z=\left(M_{1} \backslash z\right) \vee M_{2}$. It means that $M \backslash z$ has a $U_{2,5}$ or $U_{3,5}$-minor, contradicting the fact that the result holds for any number of elements less than the size of $E(M)$. Similarly for the contraction, if $z$ is in $E\left(M_{1}\right)$, and we let $z \in Y$, then since $z$ is not in $E\left(M_{1}\right) \cap E\left(M_{2}\right)$, it follows by Proposition 2.4.5 $M / z=\left(M_{1} / z\right) \vee M_{2}$. But if $M / z$ has a $U_{2,5}$ or $U_{3,5}$-minor, then that contradicts the fact that the result holds for any number of elements less than the size of $E(M)$. Therefore we conclude that $M$ has no $U_{2,5}$ or $U_{3,5}$-minor.

To complete the proof of Theorem 5.3.1, we use a result that has been proven by M.J.Piff and D.J.A.Welsh, see [7] for more details. We also state it here for the reader's convenience. Note that the theorem makes no assumption about the ground sets being the same.

Theorem 5.3.8. Let $M_{1}$ and $M_{2}$ be matroids on $E_{1}$ and $E_{2}$ respectively. There exists $n$ such that if $M_{1}$ and $M_{2}$ are representable over a field $F$, with $|F| \geq n$, then $M_{1} \vee M_{2}$ is also representable over $F$.

Proof of Theorem 5.3.1. Since $M_{1}$ and $M_{2}$ are regular, then they are both binary and ternary, and therefore by Lemma 5.3 .7 we know that $M_{1} \vee M_{2}$ has no $U_{2,5}$ or $U_{3,5}$-minors. By Theorem 1.7.6, if we show that $M$ has no $F_{7}$ or $F_{7}^{*}$-minors, then that would complete the proof of $M$ being ternary.

Suppose now that $M_{1}$ and $M_{2}$ are both regular, such that $M_{1} \vee M_{2}$ has an $F_{7}$ or $F_{7}^{*}$-minors. Since $M_{1}$ and $M_{2}$ are regular, then by Theorem 1.7.7 they are representable over every field, and hence they are representable over the reals. It follows then by Theorem 5.3.8 that $M_{1} \vee M_{2}$ is also representable over the reals. But $F_{7}$ and $F_{7}^{*}$ are minors of $M_{1} \vee M_{2}$ by assumption. So by Proposition 1.7.2 we must have $F_{7}$ and $F_{7}^{*}$ as representable over the reals 
as well, contradicting Theorem 1.7.4. This concludes the proof for Theorem 5.3.1 that $M=M_{1} \vee M_{2}$ is a ternary matroid.

As a result of combining Theorem 5.3.1 with Theorem 3.4.1 we get the following corollary.

Corollary 5.3.9. Let $M_{1}$ and $M_{2}$ be two loopless regular matroids with $E\left(M_{1}\right) \cap E\left(M_{2}\right)=\{x, y\}$. If $M_{1}$ and $M_{2}$ are both connected, neither of them has a series pair, $\left|E\left(M_{1}\right)\right|,\left|E\left(M_{2}\right)\right| \geq 3$ and $x$ and $y$ are split in $M_{1}$ and split in $M_{2}$, then $M=M_{1} \vee M_{2}$ is a 3-connected ternary matroid.

\subsection{Decomposing ternary matroids}

The aim of this section is to prove the following theorem, which is a partial converse to Theorem 5.3.1.

Theorem 5.4.1. Let $M=M_{1} \vee M_{2}$ be a 3-connected ternary matroid, such that $\left|E\left(M_{1}\right) \cap E\left(M_{2}\right)\right|=2$. If $M_{1}$ and $M_{2}$ are 3-connected, then $M_{1}$ and $M_{2}$ are both regular matroids.

A matroid $M$ uses a set $T$, if $T \subseteq E(M)$. A class $\mathcal{N}$ of matroids is $t$-rounded, if every member of $\mathcal{N}$ is $(t+1)$-connected, and the following condition holds: If $M$ is a $(t+1)$-connected matroid having an $\mathcal{N}$-minor, from the class and $X$ is a subset of $E(M)$ with at most $t$ elements, then $M$ has an $\mathcal{N}$-minor using $X$. We are particularly interested in 2-roundedness. The following results will be used in the proof.

Proposition 5.4.2. [5, Proposition 11.2.13] Every 3-connected matroid $M$ having at least four elements has a minor isomorphic to $U_{2,4}$ or $M\left(K_{4}\right)$.

Proposition 5.4.3. [5, Corollary 11.2.14] Every 3-connected binary matroid having at least four elements has a minor isomorphic to $M\left(K_{4}\right)$.

Proposition 5.4.4. [5, Proposition 11.3.8] Let $M$ be a 3-connected matroid having a $U_{2,4}$-minor and suppose that $e$ and $f$ are distinct elements of $M$. Then $M$ has $U_{2,4}$-minor using $\{e, f\}$. 
Theorem 5.4.5. [5, Proposition 11.3.9] Let $t$ be 1 or 2 and $\mathcal{N}$ be a collection of $(t+1)$-connected matroids. Then $\mathcal{N}$ is t-rounded if and only if the following condition holds: If $M$ is a $(t+1)$-connected matroid having an $\mathcal{N}$-minor such that $|E(M)-E(N)|=1$, and $X$ is a subset of $E(M)$ with at most $t$ elements, then $M$ has an $\mathcal{N}$-minor using $X$.

The following follows from Theorem 5.4.5.

Proposition 5.4.6. [5] The collection of matroids $\left\{U_{2,4}, M\left(K_{4}\right)\right\}$ is 2rounded.

Next we prove two lemmas that will also be used in proving Theorem 5.4 .1 .

Lemma 5.4.7. Let $N$ be a connected matroid that has at least 3 elements, such that $N$ has no series pairs. If $E(N)=A \cup\{x, y\}$, then $N / A$ consists entirely of loops.

Proof. We first observe that $E(N / A)=\{x, y\}$. Let us consider what possibilities $x$ and $y$ have in $N / A$.

Case 1. $\{x, y\}$ are independent in $N / A$. Meaning that $x$ and $y$ are coloops of $N / A$. But a coloop cannot be generated by a series of contractions. It follows that $N$ must have had a coloop to start with, contradicting the fact that it is connected.

Case 2. $\{x, y\}$ is a parallel pair in $N / A$. Here there are two possibilities for $x$ and $y$ to be a parallel pair.

Case 2.1. $N$ has a minor containing three elements $x, y$ and $a$, where $\{x, y\}$ is a parallel pair, and $a$ as a coloop in this minor, as shown in Figure 5.4(a). It follows that $a$ must have been a coloop of $N$, giving a contradiction.

Case 2.2. $N$ has a minor isomorphic to $U_{2,3}$ containing the elements $x, y$ and $a$, as shown in Figure 5.4(b). It follows that $\{x, y\}$ is a series pair of this minor, and therefore must have been a series pair of $N$, giving a contradiction.

Case 3. One of $x$ and $y$ is a loop in $N / A$, and the other is not. Assume without loss of generality that $x$ is a loop in $N / A$. It follows that $N$ must have a minor where $x$ is in a parallel class, and $y$ is not. So that $y$ is a coloop of this minor, and hence a coloop of $N$, giving a contradiction. 


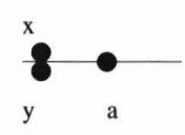

(a)

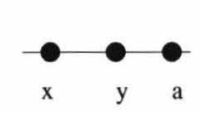

(b)

Figure 5.4: $x$ and $y$ as parallel pairs.

Therefore it follows that the only possibility left for $x$ and $y$, is for $x$ to be a loop in $N / A$ and $y$ to be a loop in $N / A$. Hence $N / A$ consists entirely of loops.

Lemma 5.4.8. Let $M=M_{1} \vee M_{2}$, such that $E\left(M_{1}\right) \cap E\left(M_{2}\right)=\{x, y\}$. If both $M_{1}$ and $M_{2}$ are connected, $\left|E\left(M_{1}\right)\right| \geq 3,\left|E\left(M_{2}\right)\right| \geq 3$, and neither of them has $\{x, y\}$ as a series pair, then $M /\left(E\left(M_{1}\right)-\{x, y\}\right)=M_{2}$. Similarly, $M /\left(E\left(M_{2}\right)-\{x, y\}\right)=M_{1}$.

Proof. Let $E\left(M_{2}\right)-\{x, y\}=A$, and $E\left(M_{1}\right)-\{x, y\}=B$. Without loss of generality, consider $M / A$. By Proposition 2.4.5 $M / A=M_{1} \vee M_{2} / A$. But $E\left(M_{2} / A\right)=\{x, y\}$. So it follows by Lemma 5.4 .7 that $M_{2} / A$ consists entirely of loops. Hence $M / A=M_{1}$. Similarly, $M / B=M_{2}$.

Now we prove Theorem 5.4.1.

Proof of Theorem 5.4.1. Let $E\left(M_{1}\right) \cap E\left(M_{2}\right)=\{x, y\}$. Since $M$ is the union of two matroids with exactly two elements in common, and $M$ is 3connected, then by Theorem 3.4.1 it follows that both $M_{1}$ and $M_{2}$ have to be connected. Moreover, each must have size at least 3, and neither of them has a series pair. It follows then by Lemma 5.4 .8 that both $M_{1}$ and $M_{2}$ are minors of $M$. Hence by Proposition 1.7.2 they are both ternary. So what we need to show now to complete the proof, is that $M_{1}$ and $M_{2}$ are both binary. Assume that both $M_{1}$ and $M_{2}$ are 3-connected. If both $M_{1}$ and $M_{2}$ are binary, then we are done. So consider the following possibilities.

Case 1.1. Only one of $M_{1}$ and $M_{2}$ is not binary. Assume without loss of generality that $M_{1}$ is not binary and $M_{2}$ is binary. By Proposition 5.4.4, $M_{1}$ has a $U_{2,4}$-minor using $x$ and $y$. So let $N_{1}=M_{1} \backslash A / B \cong U_{2,4}$ where $A$ and $B$ are subsets of $E\left(M_{1}\right)$ not containing $x$ or $y$. So that by Propositions 
2.4.1 and 2.4.5 $M \backslash A / B=\left(M_{1} \backslash A / B\right) \vee M_{2}=N_{1} \vee M_{2}$. Since $M_{2}$ is binary and 3-connected, then by Propositions 5.4.3 and 5.4.6, it must have a minor isomorphic to $M\left(K_{4}\right)$ using $x$ and $y$. Call this minor $N_{2}$. From $M\left(K_{4}\right)$ we can obtain a $U_{1,3}$-minor. It can be obtained by contracting two elements and deleting one element from $M\left(K_{4}\right)$, other than $x$ and $y$. So let $c, d \in E\left(N_{2}\right)$ be the contraction elements, and $e \in E\left(N_{2}\right)$ be the deletion element. Then $M \backslash A / B / c / d \backslash e \cong\left(U_{2,4} \vee U_{1,3}\right)$, where both $U_{2,4}$ and $U_{1,3}$ use $\{x, y\}$. But in this case $U_{2,4} \vee U_{1,3} \cong U_{3,5}$ as shown in Figure 5.5, which is not ternary, contradicting the fact that $M$ is ternary.

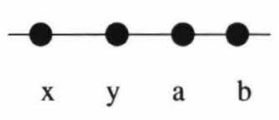

$\mathrm{U}_{2,4}$
V

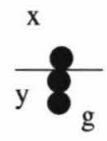

$\mathbf{U}_{1,3}$

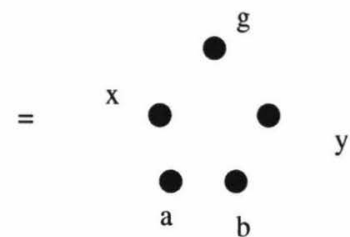

$\mathrm{U}_{2,5}$

Figure 5.5: $U_{2,5}$-minor. 
Case 1.2. Both $M_{1}$ and $M_{2}$ are not binary. By Proposition 5.4.4 both $M_{1}$ and $M_{2}$ have a $U_{2,4}$-minor using $\{x, y\}$. So that $M_{1} \backslash A / B \cong U_{2,4}$, where $A, B \subseteq E\left(M_{1}\right)$ not containing $x$ and $y$, and $M_{2} \backslash C / D \cong U_{2,4}$, where $C, D \subseteq E\left(M_{2}\right)$ not containing $x$ and $y$. By Propositions 2.4.1 and 2.4.5 $M \backslash A / B \backslash C / D=\left(U_{2,4} \vee U_{2,4}\right) \cong U_{4,6}$. But $U_{4,6}$ is not ternary, contradicting the fact that $M$ is ternary. Hence we have shown that if $M_{1}$ and $M_{2}$ are 3 -connected, then they are regular.

As noted earlier, Theorem 5.4.1 is a partial converse of Theorem 5.3.1. The next proposition is not used in this thesis, but together with Conjecture 6.1.2, which will be stated in the next chapter, makes a complete proof for a full converse of Theorem 5.3.1.

Proposition 5.4.9. Let $M$ be a non-binary connected but not 3-connected matroid, where $\{x, y\} \subseteq E(M)$. If $x$ and $y$ are split in $M$, then $M$ has a $U_{2,4}$-minor using $x$ and $y$.

Proof. The proof is by induction, first we prove the theorem is true for the base case when $|E(M)|=4$.

Base case step. If $|E(M)|=4$, then since $\{x, y\} \subseteq E(M)$, and $M$ has a $U_{2,4}$-minor, then $M \cong U_{2,4}$ using $x$ and $y$.

Inductive step. Assume now that $|E(M)|=n \geq 5$, and assume that the theorem holds for all $|E(M)| \lesseqgtr n$. If $M$ has a 2-separation $(A, B)$ that splits $x$ and $y$, then let us assume without loss of generality that $x \in A$ and $y \in B$. Let $M_{A}$ and $M_{B}$ be two matroids such that $M=M_{A} \oplus_{2} M_{B}$. By Proposition 1.8.21 there must exist an intersection element between $M_{A}$ and $M_{B}$. So let $E\left(M_{A}\right) \cap E\left(M_{B}\right)=\{p\}$ be the base-point. By property of 2-sum $M_{A} \backslash p=M \mid A$ and $M_{B} \backslash p=M \mid B$. If both $M_{A}$ and $M_{B}$ are binary, then by Proposition 1.8.11, their 2-sum, which is $M$, is also binary, giving a contradiction. So at least one of $M_{A}$ and $M_{B}$ is non-binary. Assume without loss of generality that $M_{B}$ is non-binary. If $M_{B}$ has a $U_{2,4}$-minor using $x$ and $y$, then we are done, so assume that is does not have a $U_{2,4}$-minor using $x$ and $y$.

Consider $M_{B}$. Replace element $p$ by $x$ in $M_{B}$, and call the resulting matroid $N . N$ is a minor of $M$ isomorphic to $M_{B}$. So $N$ is non-binary. If 
$N$ is 3 -connected, then by Proposition 5.4 .4 it has a $U_{2,4}$-minor using $x$ and $y$ and we are done. So let us suppose that it is not 3 -connected, so that it has a 2-separation $(P, Q)$. If every 2-separation in $N$ splits $x$ and $y$, then the inductive argument implies that $N$ has a $U_{2,4}$-minor using $x$ and $y$ and we are done. So let us suppose that $x$ and $y$ are not split in $N$. Without loss of generality assume that both $x, y \in P$.

Now replace $x$ by $p$ in $N$. The resulting matroid is $M_{B}$ again like it was at the start. The difference now, however, is that we know that $M_{B}$ has a 2-separation such that $p$ and $y$ are not split in $M_{B}$. Suppose $M_{B}$ has a 2-separation $\left(P^{\prime}, Q\right)$, such that both $p, y \in P^{\prime}$. Then let $M\left(P^{\prime}\right)$ and $M(Q)$ be two matroids such that $M_{B}=M\left(P^{\prime}\right) \oplus_{2} M(Q)$, and let $E\left(M\left(P^{\prime}\right)\right) \cap$ $E(M(Q))=\{z\}$ be the base-point, so that $P^{\prime} \cup\{z\}=E\left(M\left(P^{\prime}\right)\right), Q \cup\{z\}=$ $E(M(Q))$. We have the following:

$$
M=M_{A} \oplus_{2}\left(M\left(P^{\prime}\right) \oplus_{2} M(Q)\right)
$$

Let $(Q, R)$ be a partition of $M$, such that $R=P^{\prime}-\{p\} \cup A$. Due to the fact that the 2-sum of $M_{A}$ and $M_{B}$ was applied between $M_{A}$ and the set $P^{\prime}$ which contains $p$, we get

$$
M_{A} \oplus_{2}\left(M\left(P^{\prime}\right) \oplus_{2} M(Q)\right)=\left(M_{A} \oplus_{2} M\left(P^{\prime}\right)\right) \oplus_{2} M(Q) .
$$

Where $E\left(M_{A} \oplus_{2} M\left(P^{\prime}\right)\right)=R \cup\{z\}$. Therefore $(R, Q)$ is a 2-separation of $M$. Moreover, $x$ and $y$ are both in the same part of the 2 -separation $R$. But that contradicts the assumption that $M$ has no 2-separation that does not split $x$ and $y$. Therefore $N$ must split $x$ and $y$. Since $|E(N)| \lesseqgtr n$, then $N$ has a $U_{2,4}$-minor using $x$ and $y$. Since this was the case for $|E(M)|=n$, then the theorem holds for all values of $n$ by induction. 


\section{Chapter 6}

\section{What is next?}

\subsection{Conjectures}

In this chapter, we state some conjectures that we believe are true, but did not spend a lot of time thinking about due to severe time constraints. To resolve these issues would strengthen some of the results presented in the preceding chapters.

\section{Chapter 5: Decomposable ternary matroids}

Section 5.2. Theorem 5.2.1 shows that if a matroid $M$ is decomposable, then it must have at least two non-fixed elements $p$ and $q$. The next conjecture is the converse of Theorem 5.2.1.

Conjecture 6.1.1. Let $M$ be a 3-connected ternary matroid that has two non-fixed elements $p$ and $q$. Let $(A, B)$ be a separation of $M \backslash p, q$. Then $M$ is decomposable such that $M=(M / A) \vee(M / B)$.

Section 5.4. Theorem 5.4.1 has shown that a 3-connected ternary matroid decomposes into two regular matroid, provided that the two matroids are 3 -connected. To be able to have a stronger version of the theorem, the following conjecture is needed.

Conjecture 6.1.2. Let $M$ be a connected but not 3-connected binary matroid. If $x$ and $y$ are split in $M$, where $\{x, y\} \subseteq E(M)$, then $M$ has a minor isomorphic to the matroid shown in Figure 6.1. 


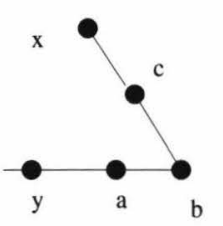

Figure 6.1: Minor of a binary matroid that has $x$ and $y$ spilt.

If the above conjecture is true, then by combining it with Proposition 5.4 .9 , we can prove the following conjecture, which is a full converse of Theorem 5.3.1.

Conjecture 6.1.3. Let $M=M_{1} \vee M_{2}$ be a 3-connected ternary matroid, such that $\left|E\left(M_{1}\right) \cap E\left(M_{2}\right)\right|=2$. Then both $M_{1}$ and $M_{2}$ are regular matroids. 


\section{Bibliography}

[1] Cunningham, W. (1979). Binary matroid sums. Quart. J. Math. Oxford Ser. (2). 30, 271-281.

[2] Geelen, James. Oxley, James. Vertigan, Dirk and Whittle, Geoff. (1998). Weak maps and stabilizers of classes of matroids. Advances in Applied Mathematics 21, 305-341.

[3] Geelen, James. Oxley, James. Vertigan, Dirk and Whittle, Geoff. (2002). Totally free expansions of matroids. Journal of Combinatorial Theory, Series B 84, 130-179.

[4] Kung, Joseph. (1986). A source book in matroid theory. Birkhäuser, Boston.

[5] Oxley, J. (1992). Matroid theory. Oxford University Press, New York.

[6] Oxley, J. (November 22, 2006). What is a matroid?, 35.

[7] Piff, M.J and Welsh, D.J.A. (1970). On the vector representation of matroids, J. London Math. Soc. 2, 284-288.

[8] Recski, A; Lovas, L. (1973). On the sums of matroids. Acta mathematica Academia Scientiarum Hungaricae. 24/3-4, 329-333.

[9] Recski, A. (1976). On the sums of matroids II. Proceedings of the Fifth British Combinatorial Conference, University of Aberdeen. 15, 515-520.

[10] Recski, A. (1981). On the sums of matroids III. Discrete mathematics. 36, 273-287. 
[11] White, N. (1992). Theory of matroids. Cambridge University Press, Cambridge. 


\section{Index}

$F$-representable, 2

$t$-rounded, 73

$k$-separation, 10

$n$-connected, 11

1-sum, 5

2-roundedness, 73

2-sum

operation, 12

parts, 12

associativity, 22

automorphism, 53

base-point, 9

basis, 1,3

of the union, 24

of direct sum, 6

binary, 8, 9

bipartite graph, 23,25

characteristic, 8

circuit elimination axiom, 2

circuits

of a matroid, 1

of direct sum, 5

of parallel connection, 9

of series connection, 9

of the union, 23

clones, 53 closed

under 2-sum, 13

under direct sum, 8

closure operator

of direct sum, 7

of the union, 23

cocircuit, 3

in the union, 44

cofixed, 53

cohyperplane, 3

coindependent set, 3

commutativity, 22

connectivity, 10

connectivity function, 16

contraction, 3

cospanning set, 3

decomposable, 20

decomposition

of matroids, 20

deletion, 2

dependent, 1

direct sum, 5,8

disconnected, 11

dual, 3

connected, 5

extension, 37 
Fano matroid, 8

fixed, 53

flat, 7

of direct sum, 7

graphic matroid, 2, 25

ground set, 1

ground sets

of the union, 20

indecomposable, 20

independence augmentation axiom, 1

independent sets, 1

irreducible, 20

isomorphism, 54

matching, 25

matroid, 1

connected, 4

minor, 3,8

pair

of coloops, 53

of loops, 53

parallel

connection, 9

extension, 37

pair, 53

partial transversal, 25

partition, 10

rank, 2

matroid union, 34

of direct sum, 6

rank function, 2

of the union, 22 reducible, 20

regular, 8,9

representable, 8

representable over $F, 2$

restriction, 2

series

connection, 9

pair, 53

single-element extensions, 37

split, 42

ternary, 8, 9

transversal, 8, 25, 26

truncation, 34

union, 20

universal stabilizer, 57

vector matroid, 2

well-closed class, 56 